\title{
Planar Tetra-, Penta-, Hexa-, Hepta-, and Octacoordinate Silicons: A Universal Structural Pattern
}

\author{
Si-Dian Li*, Chang-Qing Miao, Jin-Chang Guo, Guang-Ming Ren
}

Institute of Materials sciences and Department of Chemistry, Xinzhou Teachers' University, Xinzhou 034000, Shanxi, P. R. China and Institute of Molecular Science, Shanxi University, Taiyuan 030001, Shanxi, P. R. China.

\section{Supporting Information}


Part I: Optimized Coordinates, vibrational frequencies, NBO charges and Wiberg bond indices of $\mathrm{B}_{\mathrm{n}} \mathrm{E}_{2} \mathrm{X}$ neutrals and anions

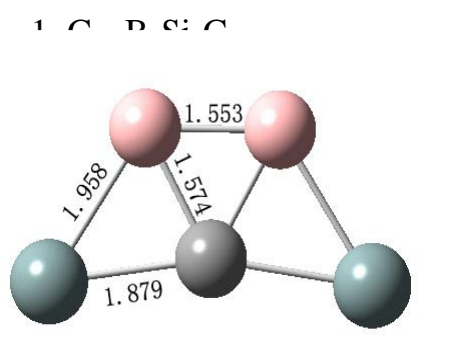

$\begin{array}{lccccc} & \mathrm{X} / \AA & \mathrm{Y} / \AA & \mathrm{Z} / \AA & \mathrm{q} / \mathrm{e} & \text { WBI } \\ \mathrm{C} & 0.000000 & 0.000000 & 0.145381 & -1.44 & 3.38 \\ \mathrm{Si} & 0.000000 & 1.861258 & 0.405918 & +0.85 & 1.78 \\ \mathrm{~B} & 0.000000 & 0.776590 & -1.223798 & -0.13 & 3.22 \\ \mathrm{~B} & 0.000000 & -0.776590 & -1.223798 & -0.13 & 3.22 \\ \mathrm{Si} & 0.000000 & -1.861258 & 0.405918 & +0.85 & 1.78\end{array}$

$\begin{array}{rrrrrrr}\text { Vibrational frequencies } / \mathrm{cm}^{-1} & 193 & 213 & 281 & 542 & 573 & 674 \\ 951 & & 1017 & 1238 & & & \end{array}$

Wiberg bond Index matrix

\begin{tabular}{|c|c|c|c|c|c|}
\hline tom & 1 & 2 & 3 & 4 & 5 \\
\hline & & & --- & & \\
\hline . C & 0.0000 & 0.6890 & 1.0028 & 1.0028 & 0.68 \\
\hline SI & 0.6890 & 0.0000 & 0.9144 & 0.1208 & 0.0565 \\
\hline B & 1.0028 & 0.9144 & 0.0000 & 1.1824 & .1208 \\
\hline B & 1.0028 & 0.1208 & 1.1824 & 0.0000 & 0.9144 \\
\hline 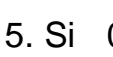 & 0.6890 & 0.0565 & 8 & 9144 & 0.000 \\
\hline
\end{tabular}

2. $\mathrm{C}_{2 \mathrm{v}} \mathrm{B}_{2} \mathrm{Si}_{2} \mathrm{C}^{-}$

$\begin{array}{lllllll} & & \mathrm{X} / \AA & \mathrm{Y} / \AA & \mathrm{Z} / \AA & \mathrm{q} / \mathrm{e} & \mathrm{WBI} \\ \mathrm{C} & 0.000000 & 0.000000 & 0.155746 & -1.28 & 3.49 \\ \mathrm{Si} & 0.000000 & 1.876823 & 0.393351 & +0.37 & 2.02 \\ \mathrm{~B} & 0.000000 & 0.790783 & -1.194831 & -0.23 & 3.30 \\ \mathrm{~B} & 0.000000 & -0.790783 & -1.194831 & -0.23 & 3.30 \\ \mathrm{Si} & 0.000000 & -1.876823 & 0.393351 & +0.37 & 2.02\end{array}$

$\begin{array}{rllllll}\text { Vibrational frequencies } / \mathrm{cm}^{-1} & 269 & 275 & 303 & 518 & 565 & 655 \\ 916 & 965 & 1172 & & & & \end{array}$

Wiberg bond Index matrix

$\begin{array}{lccccc}\text { Atom } & 1 & 2 & 3 & 4 & 5 \\ --- & ---- & ---- & ---- & ---- & \\ \text { 1. C } & 0.0000 & 0.7248 & 1.0210 & 1.0210 & 0.7248 \\ \text { 2. Si } & 0.7248 & 0.0000 & 1.0648 & 0.0964 & 0.1358 \\ \text { 3. B } & 1.0210 & 1.0648 & 0.0000 & 1.1173 & 0.0964 \\ \text { 4. B } & 1.0210 & 0.0964 & 1.1173 & 0.0000 & 1.0648 \\ \text { 5. Si } & 0.7248 & 0.1358 & 0.0964 & 1.0648 & 0.0000\end{array}$


3. $\mathrm{C}_{2 \mathrm{v}} \mathrm{B}_{2} \mathrm{Si}_{2} \mathrm{Si}$

$\begin{array}{lcccccc} & & \mathrm{X} / \AA & \mathrm{Y} / \AA & \mathrm{Z} / \AA & \mathrm{q} / \mathrm{e} & \text { WBI } \\ & \mathrm{Si} & 0.000000 & 0.000000 & 0.835817 & +0.11 & 2.99 \\ \mathrm{Si} & 0.000000 & 2.323426 & -0.018200 & +0.61 & 2.12 \\ \mathrm{~B} & 0.000000 & 0.763175 & -1.119183 & -0.66 & 3.72 \\ \mathrm{~B} & 0.000000 & -0.763175 & -1.119183 & -0.66 & 3.72 \\ & \mathrm{Si} & 0.000000 & -2.323426 & -0.018200 & +0.61 & 2.12\end{array}$

$\begin{array}{lllllll}\text { Vibrational frequencies } / \mathrm{cm}^{-1} & 68 & 106 & 201 & 357 & 366 & 510\end{array}$ $\begin{array}{lll}607 & 766 \quad 1338\end{array}$

Wiberg bond Index matrix
$\begin{array}{lllrrr}\text { Atom } 1 & 2 & 3 & 4 & 5 \\ - & & & \end{array}$
1. Si $0.0000 \quad 0.6532 \quad 0.8409 \quad 0.8409 \quad 0.6532$
2. Si $0.6532 \quad 0.0000 \quad 1.1657 \quad 0.1467 \quad 0.1548$
3. B $\quad 0.8409 \quad 1.1657 \quad 0.0000 \quad 1.5659 \quad 0.1467$
4. $\begin{array}{lllllll}\text { B } & 0.8409 & 0.1467 & 1.5659 & 0.0000 & 1.1657\end{array}$
$\begin{array}{llllll}\text { 5. Si } & 0.6532 & 0.1548 & 0.1467 & 1.1657 & 0.0000\end{array}$

4. $\mathrm{C}_{2 \mathrm{v}} \mathrm{B}_{2} \mathrm{Si}_{2} \mathrm{Si}^{-}$

$\begin{array}{lllllll}\mathrm{X} / \AA & \mathrm{Y} / \AA & \mathrm{Z} / \AA & \mathrm{q} / \mathrm{e} & \text { WBI } \\ \mathrm{Si} & 0.000000 & 0.000000 & 0.813007 & +0.16 & 3.02 \\ \mathrm{Si} & 0.000000 & 2.320183 & -0.013419 & +0.14 & 2.27 \\ \mathrm{~B} & 0.000000 & 0.778182 & -1.100637 & -0.72 & 3.71 \\ \mathrm{~B} & 0.000000 & -0.778182 & -1.100637 & -0.72 & 3.71 \\ \mathrm{Si} & 0.000000 & -2.320183 & -0.013419 & +0.14 & 2.27\end{array}$

$\begin{array}{rrrrrrrr}\text { Vibrational frequencies } / \mathrm{cm}^{-1} & 110 & & 201 & 289 & 316 & 377 & 539 \\ 628 & & 773 & 1251 & & & \end{array}$

Wiberg bond Index matrix

$\begin{array}{lccccc}\text { Atom } & 1 & 2 & 3 & 4 & 5 \\ \text { 1. Si } & 0.0000 & 0.6699 & 0.8379 & 0.8379 & 0.6699 \\ \text { 2. Si } & 0.6699 & 0.0000 & 1.3770 & 0.0676 & 0.1588 \\ \text { 3. B } & 0.8379 & 1.3770 & 0.0000 & 1.4255 & 0.0676 \\ \text { 4. B } & 0.8379 & 0.0676 & 1.4255 & 0.0000 & 1.3770 \\ \text { 5. Si } & 0.6699 & 0.1588 & 0.0676 & 1.3770 & 0.0000\end{array}$


5. $\mathrm{C}_{2 \mathrm{v}} \mathrm{B}_{2} \mathrm{Si}_{2} \mathrm{Ge}$

$\begin{array}{lllllll} & & \mathrm{X} / \AA & \mathrm{Y} / \AA & \mathrm{Z} / \AA & \mathrm{q} / \mathrm{e} & \mathrm{WBI} \\ \mathrm{Ge} & 0.000000 & 0.000000 & 0.726866 & +0.15 & 2.73 \\ \mathrm{Si} & 0.000000 & 2.360351 & -0.341229 & +0.59 & 2.14 \\ \mathrm{~B} & 0.000000 & 0.765205 & -1.370531 & -0.66 & 3.73 \\ \mathrm{~B} & 0.000000 & -0.765205 & -1.370531 & -0.66 & 3.73 \\ \mathrm{Si} & 0.000000 & -2.360351 & -0.341229 & +0.59 & 2.14\end{array}$

$\begin{array}{rrrrrrr}\text { Vibrational frequencies } / \mathrm{cm}^{-1} & 64 & 157 & 166 & 252 & 296 & 452 \\ 553 & 782 & 1352 & & & & \end{array}$

Wiberg bond Index matrix

\begin{tabular}{|c|c|c|c|c|c|}
\hline Atom & 1 & 2 & 3 & 4 & 5 \\
\hline--- & ----- & $---\cdot$ & ------ & ---- & \\
\hline Ge & 0.0000 & 0.5791 & 0.7850 & 0.7850 & 0.5791 \\
\hline 2. $\mathrm{Si}$ & 0.5791 & 0.0000 & 1.2297 & 0.1489 & 0.1872 \\
\hline 3. B & 0.7850 & 1.2297 & 0.0000 & 1.5642 & 0.1489 \\
\hline 4. $B$ & 0.7850 & 0.1 & 1.5642 & 0.0000 & 1.2297 \\
\hline 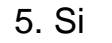 & .5791 & 0.1872 & 0.1489 & 1.2297 & 0.0000 \\
\hline
\end{tabular}

6. $\mathrm{C}_{2 \mathrm{v}} \mathrm{B}_{2} \mathrm{Si}_{2} \mathrm{Ge}^{-}$

$\begin{array}{lllllll} & & \mathrm{X} / \AA & \mathrm{Y} / \AA & \mathrm{Z} / \AA & \mathrm{q} / \mathrm{e} & \mathrm{WBI} \\ \mathrm{Ge} & 0.000000 & 0.000000 & 0.706097 & +0.16 & 2.78 \\ \mathrm{Si} & 0.000000 & 2.365226 & -0.328879 & +0.14 & 2.27 \\ \mathrm{~B} & 0.000000 & 0.780098 & -1.338651 & -0.72 & 3.71 \\ \mathrm{~B} & 0.000000 & -0.780098 & -1.338651 & -0.72 & 3.71 \\ \mathrm{Si} & 0.000000 & -2.365226 & -0.328879 & +0.14 & 2.27\end{array}$

$\begin{array}{lllllll}\text { Vibrational frequencies } / \mathrm{cm}^{-1} & 100 & 168 & 204 & 299 & 315 & 482\end{array}$

Wiberg bond Index matrix

\begin{tabular}{|c|c|c|c|c|c|}
\hline Atom & 1 & 2 & 3 & 4 & 5 \\
\hline--- & -- ----- & ----- & ----- - - & --- & \\
\hline 1. $\mathrm{G} \in$ & 0.0000 & 0.5917 & 0.7978 & 0.7978 & 0.5917 \\
\hline
\end{tabular}

2. Si $0.5917 \quad 0.0000 \quad 1.4354 \quad 0.0610 \quad 0.1825$

3. B $0.7978 \quad 1.4354 \quad 0.0000 \quad 1.4115 \quad 0.0610$

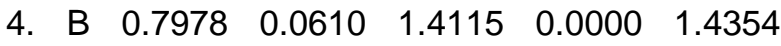

5. Si $0.5917 \quad 0.1825 \quad 0.0610 \quad 1.4354 \quad 0.0000$ 
7. $\mathrm{C}_{2 \mathrm{v}} \mathrm{B}_{2} \mathrm{Si}_{2} \mathrm{~B}^{-}$

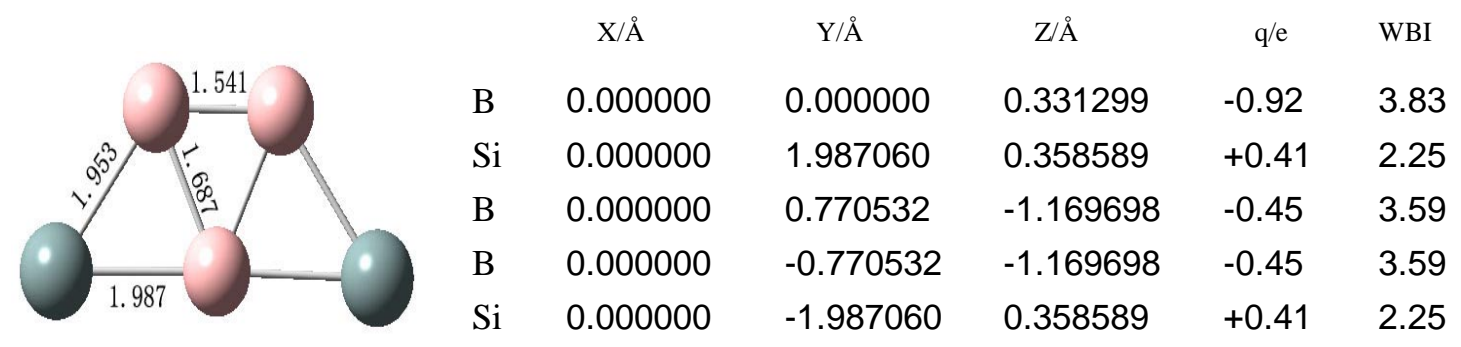

$\begin{array}{rllllll}\text { Vibrational frequencies } / \mathrm{cm}^{-1} & -9 & 153 & 282 & 515 & 534 & 651 \\ 866 & 945 & 1239 & & & & \end{array}$

Wiberg bond Index matrix

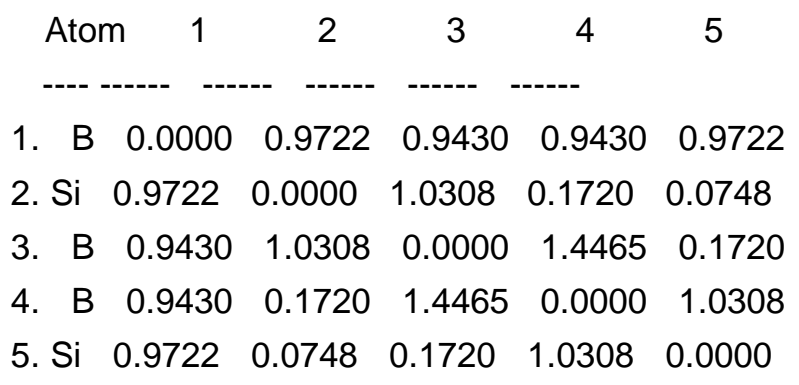

8. $\mathrm{C}_{2 \mathrm{v}} \mathrm{B}_{2} \mathrm{Si}_{2} \mathrm{Al}^{-}$

\begin{tabular}{llccccr} 
& \multicolumn{1}{c}{$\mathrm{X} / \AA$} & \multicolumn{1}{c}{$\mathrm{Y} / \AA$} & \multicolumn{1}{c}{$\mathrm{Z} / \AA$} & $\mathrm{q} / \mathrm{e}$ & WBI \\
& $\mathrm{Si}$ & 0.000000 & 2.348445 & -0.089308 & +0.21 & 2.47 \\
$\mathrm{~B}$ & 0.000000 & 0.763215 & -1.120012 & -0.80 & 3.83 \\
$\mathrm{~B}$ & 0.000000 & -0.763215 & -1.120012 & -0.80 & 3.83 \\
& $\mathrm{Si}$ & 0.000000 & -2.348445 & -0.089308 & +0.21 & 2.47 \\
& $\mathrm{Al}$ & 0.000000 & 0.000000 & 1.053903 & +0.17 & 2.25
\end{tabular}

$\begin{array}{ccccccc}\text { Vibrational frequencies } / \mathrm{cm}^{-1} & 66 & 178 & 292 & 318 & 435 & 541 \\ 772 & 1085 & 1340 & & & \end{array}$

Wiberg bond Index matrix

\begin{tabular}{|c|c|c|c|c|c|}
\hline Atom & 1 & 2 & 3 & 4 & 5 \\
\hline & & & & & \\
\hline $\mathrm{Si}$ & 0.0000 & 1.4000 & 0.2051 & 0.2360 & 0.62 \\
\hline B & 1.4000 & 0.0000 & 1.7270 & 0.2051 & 0.4994 \\
\hline B & 0.2051 & 1.7270 & 0.0000 & 1.4000 & 0.4994 \\
\hline $\mathrm{Si}$ & 0.23 & 0.2051 & 1.4000 & 0.0000 & 0.6256 \\
\hline $\mathrm{Al}$ & 205 & 1994 & 1991 & 5 & 000 \\
\hline
\end{tabular}


9. $\mathrm{C}_{2 \mathrm{v}} \mathrm{B}_{2} \mathrm{Si}_{2} \mathrm{Ga}^{-}$

$\begin{array}{llccccr} & & \text { X/A } & \text { Y/A } & \text { Z/A } & \text { q/e } & \text { WBI } \\ \text { Si } & 0.000000 & 2.347277 & -0.424415 & +0.23 & 2.47 \\ \mathrm{~B} & 0.000000 & 0.764960 & -1.444539 & -0.75 & 3.82 \\ \mathrm{~B} & 0.000000 & -0.764960 & -1.444539 & -0.75 & 3.82 \\ \mathrm{Si} & 0.000000 & -2.347277 & -0.424415 & +0.23 & 2.47 \\ \mathrm{Ga} & 0.000000 & 0.000000 & 0.849323 & +0.045 & 2.02\end{array}$

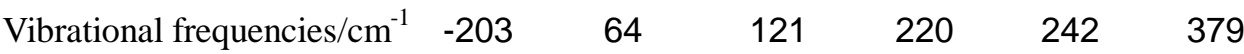

$526 \quad 786 \quad 1350$

Wiberg bond Index matrix

$\begin{array}{lccccc}\text { Atom } & 1 & 2 & 3 & 4 & 5 \\ \text { 1. } \mathrm{Si} & 0.0000 & 1.4466 & 0.2072 & 0.2904 & 0.5285 \\ \text { 2. } \mathrm{B} & 1.4466 & 0.0000 & 1.6900 & 0.2072 & 0.4808 \\ \text { 3. } \mathrm{B} & 0.2072 & 1.6900 & 0.0000 & 1.4466 & 0.4808 \\ \text { 4. } \mathrm{Si} & 0.2904 & 0.2072 & 1.4466 & 0.0000 & 0.5285 \\ \text { 5. Ga } & 0.5285 & 0.4808 & 0.4808 & 0.5285 & 0.0000\end{array}$

10. $\mathrm{C}_{2 \mathrm{v}} \mathrm{B}_{2} \mathrm{Si}_{2} \mathrm{P}$

\begin{tabular}{lllllll} 
& \multicolumn{1}{c}{ X/A } & \multicolumn{1}{c}{$\mathrm{Y} / \AA$} & \multicolumn{1}{c}{$\mathrm{Z} / \AA$} & \multicolumn{1}{c}{$\mathrm{q} / \mathrm{e}$} & WBI \\
$\mathrm{Si}$ & 0.000000 & 2.315064 & 0.034195 & +0.51 & 1.91 \\
$\mathrm{~B}$ & 0.000000 & 0.785651 & -1.107042 & -0.53 & 3.51 \\
$\mathrm{~B}$ & 0.000000 & -0.785651 & -1.107042 & -0.53 & 3.51 \\
$\mathrm{Si}$ & 0.000000 & -2.315064 & 0.034195 & +0.51 & 1.91 \\
$\mathrm{P}$ & 0.000000 & 0.000000 & 0.674197 & +0.033 & 3.22
\end{tabular}

$\begin{array}{lllllll}\text { Vibrational frequencies } / \mathrm{cm}^{-1} & 106 & 198 & 229 & 286 & 392 & 547\end{array}$

Wiberg bond Index matrix

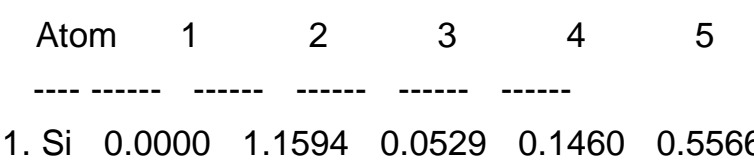

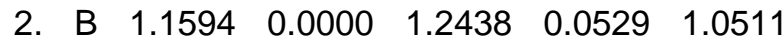

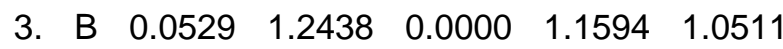

4. Si $0.1460 \quad 0.0529 \quad 1.1594 \quad 0.0000 \quad 0.5566$

$\begin{array}{lllllll}\text { 5. } & P & 0.5566 & 1.0511 & 1.0511 & 0.5566 & 0.0000\end{array}$ 
11. $\mathrm{C}_{2 \mathrm{v}} \mathrm{B}_{2} \mathrm{Si}_{2} \mathrm{As}$

$\begin{array}{lcccccc} & & \mathrm{X} / \AA & \mathrm{Y} / \AA & \mathrm{Z} & \mathrm{q} / \mathrm{A} & \mathrm{WBI} \\ \mathrm{Si} & 0.000000 & 2.384444 & -0.274996 & +0.49 & 1.94 \\ \mathrm{~B} & 0.000000 & 0.787784 & -1.308010 & -0.57 & 3.53 \\ \mathrm{~B} & 0.000000 & -0.787784 & -1.308010 & -0.57 & 3.53 \\ & \mathrm{Si} & 0.000000 & -2.384444 & -0.274996 & +0.49 & 1.94 \\ & \mathrm{As} & 0.000000 & 0.000000 & 0.629696 & +0.17 & 3.09\end{array}$

$\begin{array}{cccccccc}\text { Vibrational frequencies } / \mathrm{cm}^{-1} & 94 & 130 & 179 & 296 & 337 & 499 \\ 591 & 768 & 1242 & & & \end{array}$

Wiberg bond Index matrix

$\begin{array}{lccccc}\text { Atom } & 1 & 2 & 3 & 4 & 5 \\ \text { 1. Si } & 0.0000 & 1.2161 & 0.0418 & 0.1501 & 0.5291 \\ \text { 2. B } & 1.2161 & 0.0000 & 1.2568 & 0.0418 & 1.0156 \\ \text { 3. B } & 0.0418 & 1.2568 & 0.0000 & 1.2161 & 1.0156 \\ \text { 4. Si } & 0.1501 & 0.0418 & 1.2161 & 0.0000 & 0.5291 \\ \text { 5. As } & 0.5291 & 1.0156 & 1.0156 & 0.5291 & 0.0000\end{array}$

12. $\mathrm{C}_{2 \mathrm{v}} \mathrm{B}_{4} \mathrm{H}_{2} \mathrm{Si}$

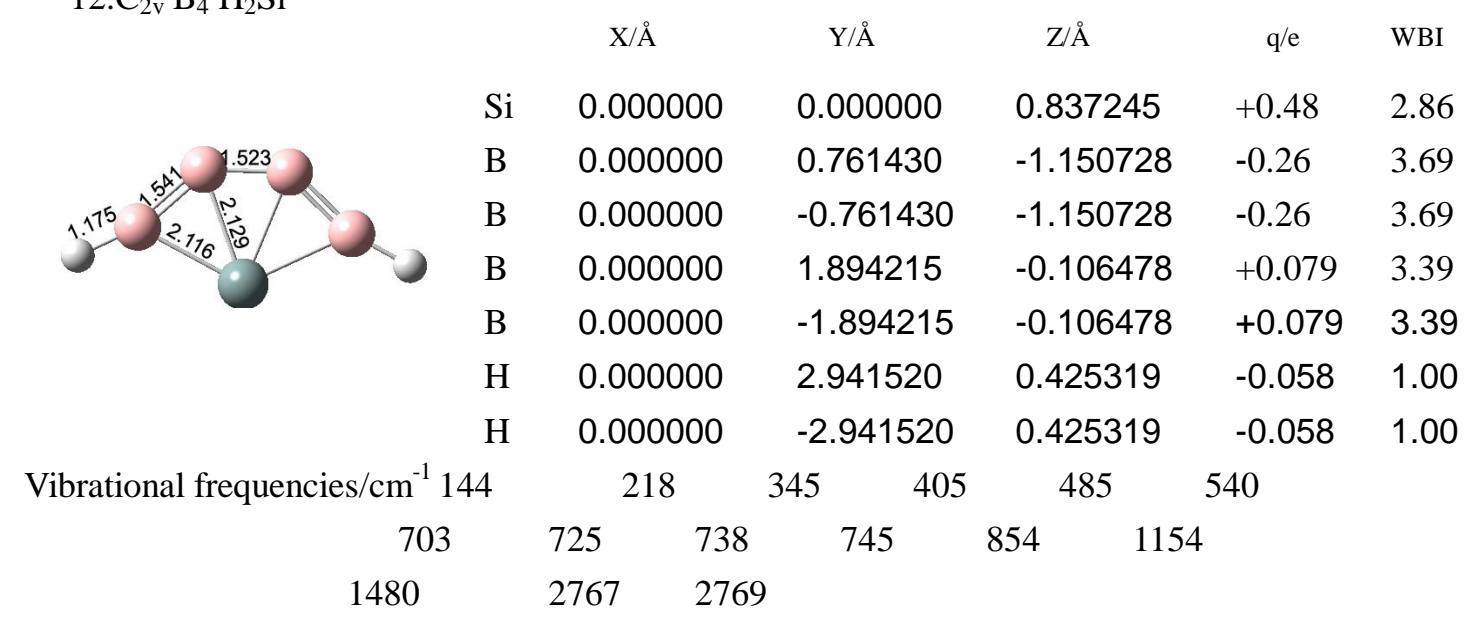

Wiberg bond Index matrix

\begin{tabular}{|c|c|c|c|c|c|c|c|}
\hline$A t$ & 1 & 2 & 3 & 4 & 5 & 6 & 7 \\
\hline & 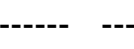 & 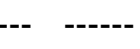 & . & . & . & & \\
\hline $\mathrm{Si}$ & 0.0000 & 0.6828 & 0.6828 & 0.7397 & 0.7397 & 0.0081 & 0.0081 \\
\hline B & 0.6828 & 0.0000 & 1.4776 & 1.3674 & 0.1502 & 0.0047 & 0.0062 \\
\hline$B$ & 0.6828 & 1.4776 & 0.0000 & 0.1502 & 1.3674 & 0.0062 & 0.0047 \\
\hline$B$ & 0.7397 & 1.3674 & 0.1502 & 0.0000 & 0.1491 & 0.9809 & 0.0038 \\
\hline$B$ & 0.7397 & 0.1502 & 1.3674 & 0.1 & 0.0000 & 0.0038 & 0.9809 \\
\hline $\mathrm{H}$ & 0.0081 & 0.0047 & 0.0062 & 0.9809 & 0.0038 & 0.0000 & 0.0001 \\
\hline $\mathrm{H}$ & 0.0081 & 0.0062 & 0.0047 & 0.0038 & 0.9809 & 0.0001 & 0.0000 \\
\hline
\end{tabular}


13. $\mathrm{C}_{2 \mathrm{v}} \mathrm{B}_{2} \mathrm{C}_{2} \mathrm{H}_{2} \mathrm{Si}$

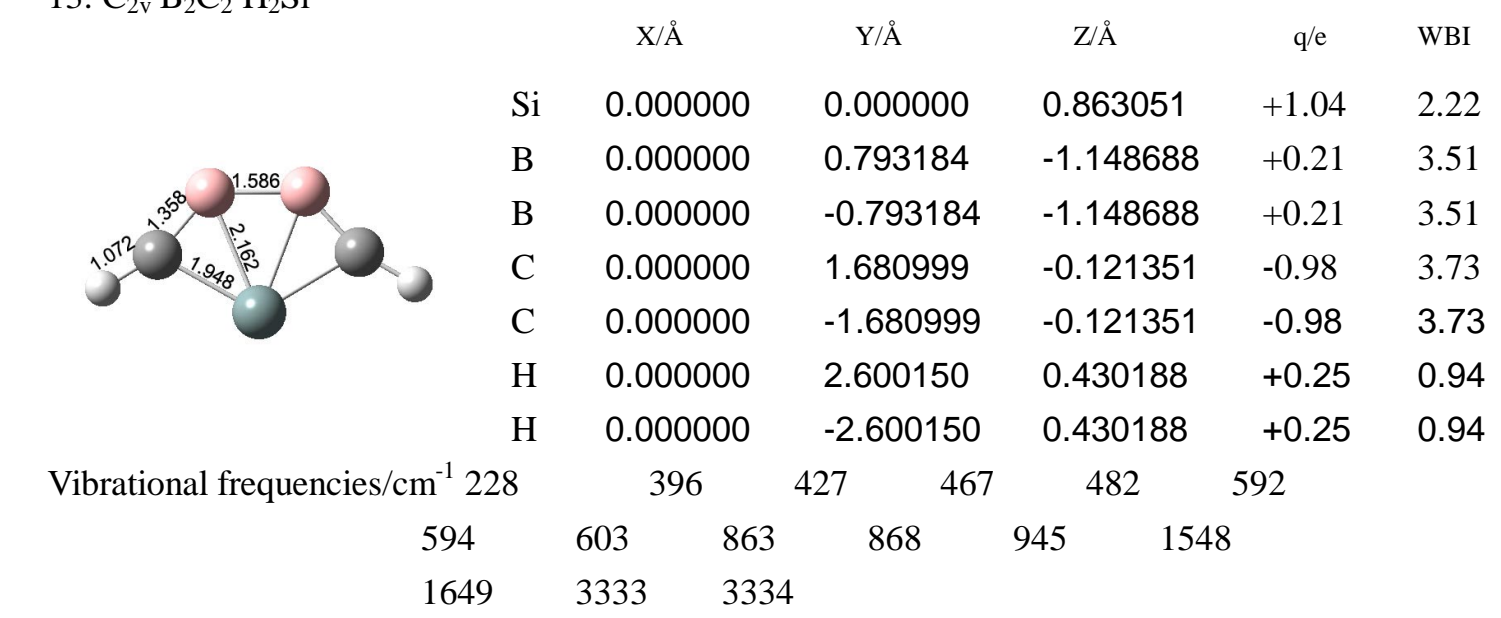

Wiberg bond Index matrix

$\begin{array}{lccccccccc}\text { Atom } & 1 & 2 & 3 & 4 & 5 & 6 & 7 \\ \text { - } & & 2 & & & & & \\ \text { 1. Si } & 0.0000 & 0.4178 & 0.4178 & 0.6821 & 0.6821 & 0.0125 & 0.0125 \\ \text { 2. } & \text { B } & 0.4178 & 0.0000 & 1.1312 & 1.9188 & 0.0240 & 0.0123 & 0.0073 \\ \text { 3. } & \text { B } & 0.4178 & 1.1312 & 0.0000 & 0.0240 & 1.9188 & 0.0073 & 0.0123 \\ \text { 4. } & \text { C } & 0.6821 & 1.9188 & 0.0240 & 0.0000 & 0.1989 & 0.9045 & 0.0051 \\ \text { 5. } & \mathrm{C} & 0.6821 & 0.0240 & 1.9188 & 0.1989 & 0.0000 & 0.0051 & 0.9045 \\ \text { 6. } & \mathrm{H} & 0.0125 & 0.0123 & 0.0073 & 0.9045 & 0.0051 & 0.0000 & 0.0000\end{array}$

14. $\mathrm{C}_{\mathrm{s}} \mathrm{B}_{3} \mathrm{C} \mathrm{H}_{2} \mathrm{Si}$

7. $\begin{array}{llllllll}H & 0.0125 & 0.0073 & 0.0123 & 0.0051 & 0.9045 & 0.0000 & 0.0000\end{array}$

\begin{tabular}{|c|c|c|c|c|c|c|c|}
\hline \\
\hline & & \multicolumn{2}{|c|}{$\mathrm{X} / \AA ̊$} & Y/Å & Z/Å & $q / e$ & WBI \\
\hline & $\mathrm{Si}$ & \multicolumn{2}{|c|}{0.000000} & 0.862518 & 0.000000 & +0.80 & 2.36 \\
\hline & B & \multicolumn{2}{|c|}{0.762972} & -1.165725 & 0.000000 & -0.32 & 3.44 \\
\hline & B & \multicolumn{2}{|c|}{-0.762972} & -1.094018 & 0.000000 & +0.17 & 3.51 \\
\hline & B & \multicolumn{2}{|c|}{1.979166} & -0.279248 & 0.000000 & +0.049 & 3.14 \\
\hline & $\mathrm{C}$ & \multicolumn{2}{|c|}{-1.679864} & -0.024610 & 0.000000 & -0.88 & 3.51 \\
\hline & $\mathrm{H}$ & \multicolumn{2}{|c|}{2.973891} & 0.342379 & 0.000000 & -0.061 & 1.00 \\
\hline & $\mathrm{H}$ & \multicolumn{2}{|c|}{-2.655940} & 0.424974 & 0.000000 & +0.24 & 0.95 \\
\hline \multirow[t]{3}{*}{ Vibrational frequencies $/ \mathrm{cm}^{-1}$} & $1 \quad 178$ & \multicolumn{2}{|c|}{227} & 384 & 543 & \multicolumn{2}{|l|}{594} \\
\hline & 604 & 637 & 746 & 833 & 899 & & \\
\hline & 1518 & 2786 & 3298 & & & & \\
\hline
\end{tabular}

Wiberg bond Index matrix

\begin{tabular}{|c|c|c|c|c|c|c|c|}
\hline om & 1 & 2 & 3 & 4 & 5 & 7 & \\
\hline & 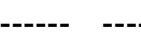 & --- & --- & ------ & ---- & & \\
\hline $\mathrm{Si}$ & 0.0000 & 0.5667 & 0.5028 & 0.4749 & 0.8022 & 0.0062 & 0.0115 \\
\hline B & 0. & 0 & 1.2820 & 1.5 & 482 & 0.00 & 0.0123 \\
\hline$B$ & 0.5 & 0 & 0.0000 & 0. & 33 & 0.0034 & 0.0087 \\
\hline $\mathrm{B}$ & 0.4749 & 73 & .0595 & 0.0000 & 0.0849 & 0.9848 & 0.0057 \\
\hline
\end{tabular}




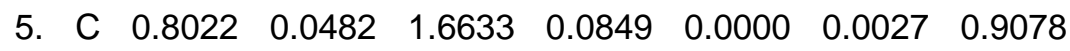
6. $\begin{array}{llllllll}\mathrm{H} & 0.0062 & 0.0059 & 0.0034 & 0.9848 & 0.0027 & 0.0000 & 0.0000\end{array}$
7. H $\begin{array}{llllllll}\text { H } & 0.0115 & 0.0123 & 0.0087 & 0.0057 & 0.9078 & 0.0000 & 0.0000\end{array}$

15. $\mathrm{C}_{2 \mathrm{v}} \mathrm{B}_{3} \mathrm{Si}_{2} \mathrm{C}$ C

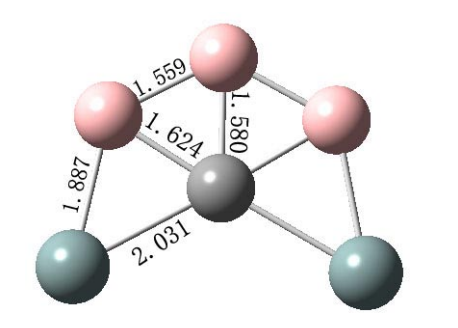

$\begin{array}{lccccc} & \mathrm{X} / \AA & \mathrm{Y} / \AA & \mathrm{Z} / \AA & \mathrm{q} / \mathrm{e} & \text { WBI } \\ \mathrm{C} & 0.000000 & 0.000000 & 0.231112 & -1.22 & 3.55 \\ \mathrm{~B} & 0.000000 & 1.380683 & 1.085695 & -0.21 & 3.15 \\ \mathrm{~B} & 0.000000 & 0.000000 & 1.810757 & +0.16 & 3.24 \\ \mathrm{~B} & 0.000000 & -1.380683 & 1.085695 & -0.21 & 3.15 \\ \mathrm{Si} & 0.000000 & -1.772203 & -0.760622 & +0.74 & 1.73 \\ \mathrm{Si} & 0.000000 & 1.772203 & -0.760622 & +0.74 & 1.73\end{array}$
$\begin{array}{lllllll}\text { Vibrational frequencies } / \mathrm{cm}^{-1} & 108 & 204 & 281 & 291 & 349 & 527\end{array}$ $\begin{array}{llllll}643 & 663 & 822 & 914 & 1116 & 1282\end{array}$

Wiberg bond Index matrix
$\begin{array}{lllrrrr}\text { Atom } & 1 & 2 & 3 & 4 & 5 & 6\end{array}$
1. C $\begin{array}{lllllll}\mathrm{C} & 0.0000 & 0.8624 & 0.8990 & 0.8624 & 0.4630 & 0.4630\end{array}$

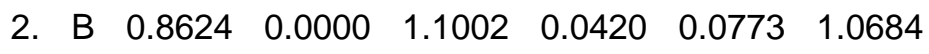

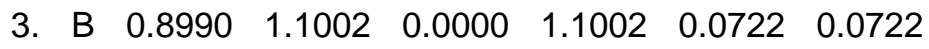

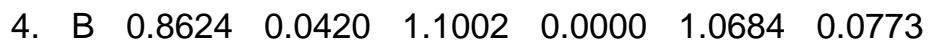

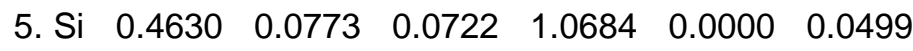
$\begin{array}{lllllll}\text { 6. Si } & 0.4630 & 1.0684 & 0.0722 & 0.0773 & 0.0499 & 0.0000\end{array}$

16. $\mathrm{C}_{2 \mathrm{v}} \mathrm{B}_{3} \mathrm{Si}_{2} \mathrm{C}^{-}$

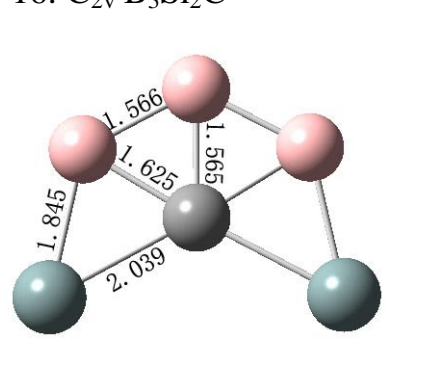

$$
\begin{array}{cc} 
& \text { X/Å } \\
\text { C } & 0.000000 \\
\text { B } & 0.000000 \\
\text { B } & 0.000000 \\
\text { B } & 0.000000 \\
\text { Si } & 0.000000 \\
\text { Si } & 0.000000
\end{array}
$$$$
\text { Y/Å }
$$$$
\text { Z/Å }
$$$$
1.389691
$$$$
\begin{aligned}
& 0.214577 \\
& 1.057192
\end{aligned}
$$$$
-1.05
$$$$
\text { WBI }
$$$$
0.000000
$$$$
1.779826
$$$$
-0.43 \quad 3.63
$$$$
-1.389691
$$$$
1.057192
$$$$
+0.11 \quad 3.27
$$$$
-1.801165
$$$$
-0.741375
$$$$
-0.43 \quad 3.63
$$$$
1.801165
$$$$
-0.741375
$$$$
+0.40
$$$$
2.37
$$

Vibrational frequencies $/ \mathrm{cm}^{-1}$

$$
601
$$

Wiberg bond Index matrix
$\begin{array}{lllllll}\text { Atom } & 1 & 2 & 3 & 4 & 5 & 6\end{array}$
1. C $\begin{array}{lllllll}\mathrm{C} & 0.0000 & 0.8776 & 0.9180 & 0.8776 & 0.4920 & 0.4920\end{array}$
2. B $\begin{array}{llllllll}0.8776 & 0.0000 & 1.0983 & 0.0903 & 0.1978 & 1.3691\end{array}$

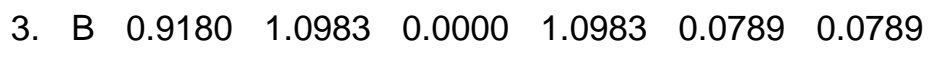

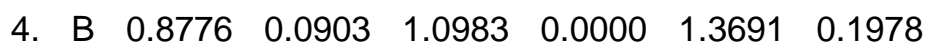
$\begin{array}{lllllll}\text { 5. Si } & 0.4920 & 0.1978 & 0.0789 & 1.3691 & 0.0000 & 0.2312\end{array}$

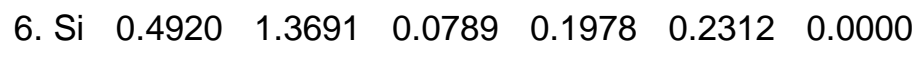




\begin{tabular}{|c|c|c|c|c|c|c|}
\hline & & $\mathrm{X} / \AA$ & $\mathrm{Y} / \AA$ & Z/Å & $q / e$ & WBI \\
\hline & $\mathrm{Si}$ & 0.000000 & 0.000000 & 0.400718 & +0.31 & 3.38 \\
\hline & B & 0.000000 & 1.440245 & -1.113758 & -0.56 & 3.48 \\
\hline & B & 0.000000 & 0.000000 & -1.683560 & -0.21 & 3.59 \\
\hline & B & 0.000000 & -1.440245 & -1.113758 & -0.56 & 3.48 \\
\hline & $\mathrm{Si}$ & 0.000000 & -2.462051 & 0.498047 & +0.50 & 2.08 \\
\hline & $\mathrm{Si}$ & 0.000000 & 2.462051 & 0.498047 & +0.50 & 2.08 \\
\hline Vibrational frequencies $/ \mathrm{cm}^{-1}$ & 100 & 117 & 177 & 359 & 364 & \\
\hline & 462 & 546 & 628 & 1090 & 1353 & \\
\hline
\end{tabular}

Wiberg bond Index matrix

18. $\mathrm{C}_{2 \mathrm{v}} \mathrm{B}_{3} \mathrm{Si}_{2} \mathrm{Si}^{-}$

\begin{tabular}{|c|c|c|c|c|c|c|}
\hline Atom & 1 & 2 & 3 & 4 & 5 & 6 \\
\hline & 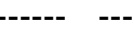 & $--\cdot-$ & - & --- & & \\
\hline 1. $\mathrm{Si}$ & 0.0000 & 0.7306 & 0.6720 & 0.7306 & 0.6235 & 0.6235 \\
\hline 2. $B$ & 0.7306 & 0.0000 & 1.3796 & 0.0524 & 0.1043 & 1.2178 \\
\hline 3. $B$ & 0.6720 & 1.3796 & 0.0000 & 1.3796 & 0.0818 & 0.0818 \\
\hline 4. $B$ & 0.7306 & 0.0524 & 1.3796 & 0.0000 & 1.2178 & 0.1043 \\
\hline 5. Si & 0.6235 & 0.1043 & 0.0818 & 1.2178 & 0.0000 & 0.0483 \\
\hline 6. $\mathrm{Si}$ & 0.6235 & 1.2178 & 0.0818 & 0.1043 & 0.0483 & 0.0000 \\
\hline
\end{tabular}

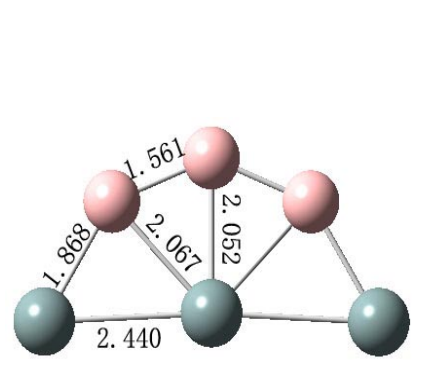

\begin{tabular}{|c|c|c|c|c|c|}
\hline & $\mathrm{X} / \AA ̊ A$ & Y/Å & Z/Å & $q / e$ & WBI \\
\hline $\mathrm{Si}$ & 0.000000 & 0.000000 & 0.382866 & +0.45 & 3.43 \\
\hline $\mathrm{B}$ & 0.000000 & 1.450315 & -1.090422 & -0.76 & 3.88 \\
\hline B & 0.000000 & 0.000000 & -1.668657 & -0.20 & 3.59 \\
\hline B & 0.000000 & -1.450315 & -1.090422 & -0.76 & 3.88 \\
\hline $\mathrm{Si}$ & 0.000000 & -2.437030 & 0.495978 & +0.13 & 2.71 \\
\hline $\mathrm{Si}$ & 0.000000 & 2.437030 & 0.495978 & +0.13 & 2.7 \\
\hline
\end{tabular}

$\begin{array}{rrrrrrrrrr}\text { Vibrational frequencies/ } \mathrm{cm}^{-1} & 126 & & 174 & & 183 & 343 & 381 & 435 \\ 464 & & 580 & 655 & 734 & 1092 & 1296\end{array}$

Wiberg bond Index matrix

\begin{tabular}{|c|c|c|c|c|c|c|}
\hline & \multirow{2}{*}{\multicolumn{6}{|c|}{ Atom 1}} \\
\hline & --- & & & & & \\
\hline Si & 0.0000 & 0.7243 & 0.6553 & 0.7243 & 0.6643 & 0. \\
\hline$B$ & 0.7243 & 0.0000 & .3737 & 0.0 & 0.1811 & 1.5675 \\
\hline B & 0.6553 & 1.3737 & 0.0000 & 1.3 & 0.0917 & 0.0917 \\
\hline B & 0.7243 & 8 & & & & 11 \\
\hline & 0.6643 & 0.1 & 0 . & 1 & 0.0 & 07 \\
\hline & U & & 17 & 0.1811 & 0.2007 & 0.0000 \\
\hline
\end{tabular}


19. $\mathrm{C}_{2 \mathrm{v}} \mathrm{B}_{3} \mathrm{Si}_{2} \mathrm{Ge}$

\begin{tabular}{|c|c|c|c|c|c|c|}
\hline & & $\mathrm{X} / \AA$ & Y/Å & Z/Å & $\mathrm{q} / \mathrm{e}$ & WBI \\
\hline & $\mathrm{Ge}$ & 0.000000 & 0.000000 & 0.426152 & +0.31 & 3.08 \\
\hline & B & 0.000000 & 1.450334 & -1.255204 & -0.58 & 3.49 \\
\hline & B & 0.000000 & 0.000000 & -1.794391 & -0.18 & 3.58 \\
\hline & B & 0.000000 & -1.450334 & -1.255204 & -0.58 & 3.49 \\
\hline & $\mathrm{Si}$ & 0.000000 & -2.571550 & 0.281683 & +0.52 & 2.04 \\
\hline & $\mathrm{Si}$ & 0.000000 & 2.571550 & 0.281683 & +0.52 & 2.04 \\
\hline Vibrational frequencies $/ \mathrm{cm}^{-1}$ & 69 & 131 & 145 & 265 & 362 & \\
\hline 37 & & 493 & 575 & 1099 & 1388 & \\
\hline
\end{tabular}

Wiberg bond Index matrix

\begin{tabular}{|c|c|c|c|c|c|c|}
\hline \multirow{2}{*}{ Atom } & 1 & 2 & 3 & 4 & 5 & 6 \\
\hline & ---- - --- & ----- & ------ & $----\quad--$ & & \\
\hline 1. $\mathrm{Ge}$ & 0.0000 & 0.6827 & 0.6234 & 0.6827 & 0.5433 & 0.5433 \\
\hline 2. $B$ & 0.6827 & 0.0000 & 1.3971 & 0.0628 & 0.0986 & 1.2513 \\
\hline 3. $B$ & 0.6234 & 1.3971 & 0.0000 & 1.3971 & 0.0805 & 0.0805 \\
\hline 4. $B$ & 0.6827 & 0.0628 & 1.3971 & 0.0000 & 1.2513 & 0.0986 \\
\hline 5. Si & 0.5433 & 0.0986 & 0.0805 & 1.2513 & 0.0000 & 0.0680 \\
\hline 6 & 0.5433 & 1.2513 & 0.0805 & 0.0986 & 0.0680 & 0.0000 \\
\hline
\end{tabular}

20. $\mathrm{C}_{2 \mathrm{v}} \mathrm{B}_{3} \mathrm{Si}_{2} \mathrm{Ge}$

\begin{tabular}{|c|c|c|c|c|c|c|}
\hline & & $\mathrm{X} / \AA ̊ \AA$ & Y/Å & Z/Å & $q / e$ & \\
\hline & B & 0.000000 & 1.463640 & -1.227786 & -0.77 & \\
\hline & B & 0.000000 & 0.000000 & -1.765667 & -0.17 & \\
\hline & B & 0.000000 & -1.463640 & -1.227786 & -0.77 & \\
\hline & $\mathrm{Si}$ & 0.000000 & -2.560405 & 0.281050 & +0.15 & \\
\hline $2 . \overline{564}$ & $\mathrm{Si}$ & 0.000000 & 2.560405 & 0.281050 & +0.15 & \\
\hline & $\mathrm{Ge}$ & 0.000000 & 0.000000 & 0.413650 & +0.41 & \\
\hline Vibrational frequencies $/ \mathrm{cm}^{-1}$ & 85 & 149 & 180 & 279 & 380 & \\
\hline 43 & & 522 & 593 & 1102 & 1338 & \\
\hline
\end{tabular}

Wiberg bond Index matrix

$\begin{array}{lllrrrr}\text { Atom } 1 & 2 & 3 & 4 & 5 & 6 \\ ------ & ---- & ----- & ----- & ---- & ---- & \end{array}$

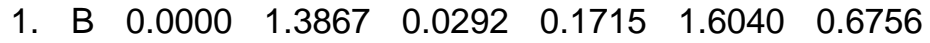

2. $\begin{array}{llllllll}\text { B } & 1.3867 & 0.0000 & 1.3867 & 0.0902 & 0.0902 & 0.6182\end{array}$

3. B $\quad 0.0292 \quad 1.3867 \quad 0.0000 \quad 1.6040 \quad 0.1715 \quad 0.6756$

4. Si $0.1715 \quad 0.0902 \quad 1.6040 \quad 0.0000 \quad 0.2222 \quad 0.5718$

5. Si $1.6040 \quad 0.0902 \quad 0.1715 \quad 0.2222 \quad 0.0000 \quad 0.5718$

6. Ge $0.6756 \quad 0.6182 \quad 0.6756 \quad 0.5718 \quad 0.5718 \quad 0.0000$ 
21. $\mathrm{C}_{2 \mathrm{v}} \mathrm{B}_{3} \mathrm{Si}_{2} \mathrm{~B}^{-}$

\begin{tabular}{|c|c|c|c|c|c|c|}
\hline & & $\mathrm{X} / \mathrm{A}$ & Y/Å & Z/Å & $q / e$ & WBI \\
\hline & B & 0.000000 & 0.000000 & 0.074554 & -0.68 & 3.83 \\
\hline & B & 0.000000 & 1.398587 & 1.088505 & -0.45 & 3.45 \\
\hline & B & 0.000000 & 0.000000 & 1.769945 & -0.17 & 3.54 \\
\hline & B & 0.000000 & -1.398587 & 1.088505 & -0.45 & 3.45 \\
\hline & $\mathrm{Si}$ & 0.000000 & -1.940492 & -0.718127 & +0.37 & 2.17 \\
\hline & $\mathrm{Si}$ & 0.000000 & 1.940492 & -0.718127 & +0.37 & 2.17 \\
\hline Vibrational frequencies $/ \mathrm{cm}^{-1}$ & 135 & 205 & 253 & 368 & 555 & \\
\hline 64 & & 843 & 856 & 1087 & 1279 & \\
\hline
\end{tabular}

Wiberg bond Index matrix

\begin{tabular}{|c|c|c|c|c|c|c|}
\hline Atom & 1 & 2 & 3 & 4 & 5 & 6 \\
\hline & 08 & 0.8264 & 0.7950 & $0 . \varepsilon$ & 9 & $0.6 s$ \\
\hline B & 4 & 0 & 4 & & & \\
\hline B & 0.7950 & 1.2744 & 0.0000 & 1.2 & 56 & 0.0956 \\
\hline B & 0.8264 & 0.0376 & 1.2744 & 0.0 & 1.2370 & 74 \\
\hline$S$ & 6910 & 4 & 56 & 1. & 0 & 7 \\
\hline Si & 6910 & 2370 & .0956 & 0.0774 & 0.0697 & 0.0000 \\
\hline
\end{tabular}

22. $\mathrm{C}_{2 \mathrm{v}} \mathrm{B}_{3} \mathrm{Si}_{2} \mathrm{Al}^{-}$

X/Å Y/Å Z/Å q/e WBI

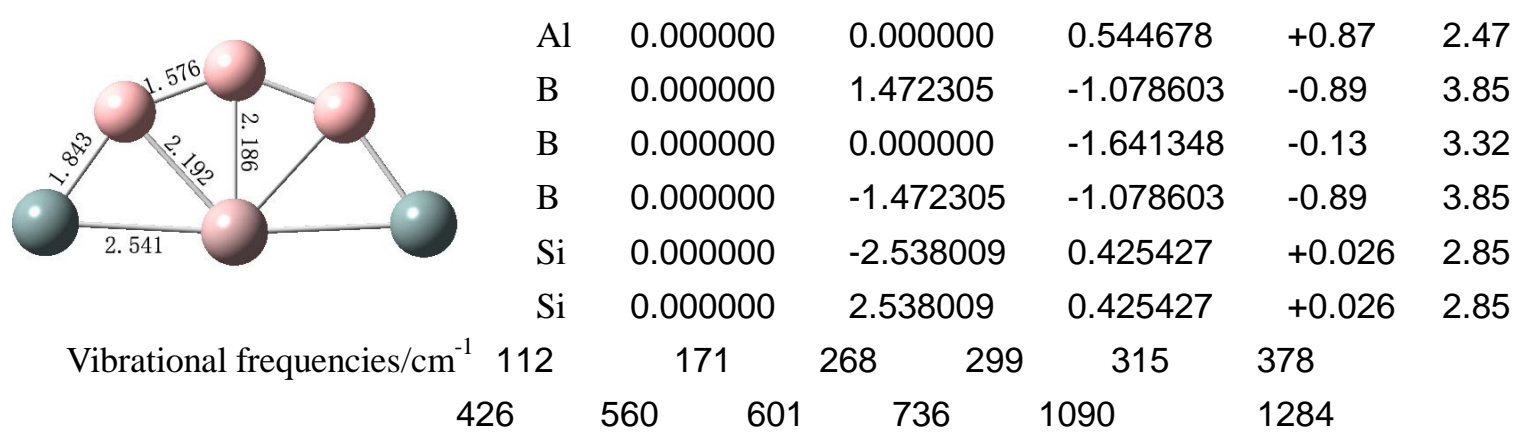

Wiberg bond Index matrix

\begin{tabular}{|c|c|c|c|c|c|c|}
\hline $\mathrm{m}$ & 1 & 2 & 3 & 4 & 5 & 6 \\
\hline & 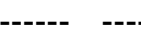 & & . & ------ & & \\
\hline $\mathrm{HI}$ & 0.0000 & 0.5094 & 0.3081 & 0.5094 & 0 & 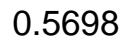 \\
\hline$B$ & 0.5094 & 0.0000 & 1.3784 & 0.0 & 0.1271 & 21 \\
\hline B & 0.3081 & 34 & .0000 & 1. & 0.1 & 0.128 \\
\hline 4. & 0.5 & 8 & 1.3784 & 0.0 & 1.8214 & 127 \\
\hline 5 & 0.5698 & 0.1271 & 0.1281 & 1.8214 & 0 & 08 \\
\hline 65 & 5698 & 1.8214 & 281 & 71 & 2008 & 0.000 \\
\hline
\end{tabular}




\begin{tabular}{|c|c|c|c|c|c|c|}
\hline & & $\mathrm{X} / \AA ̊$ & Y/Å & Z/Å & q/e & WBI \\
\hline & $\mathrm{Ga}$ & 0.000000 & 0.000000 & 0.484068 & +0.32 & 2.73 \\
\hline & B & 0.000000 & 1.442386 & -1.291653 & -0.65 & 3.61 \\
\hline & B & 0.000000 & 0.000000 & -1.846356 & -0.31 & 3.65 \\
\hline & B & 0.000000 & -1.442386 & -1.291653 & -0.65 & 3.61 \\
\hline & $\mathrm{Si}$ & 0.000000 & -2.542362 & 0.255078 & +0.14 & 2.38 \\
\hline & $\mathrm{Si}$ & 0.000000 & 2.542362 & 0.255078 & +0.14 & 2.38 \\
\hline Vibrational frequencies $/ \mathrm{cm}^{-1}$ & 75 & 81 & 147 & 263 & 366 & \\
\hline & 371 & 456 & 665 & 1087 & 1378 & \\
\hline
\end{tabular}

Wiberg bond Index matrix

\begin{tabular}{|c|c|c|c|c|c|c|}
\hline tom & 1 & 2 & 3 & 4 & 5 & 6 \\
\hline & 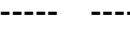 & 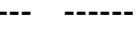 & 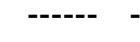 & 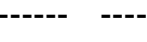 & & \\
\hline $\mathrm{Ga}$ & 0.0000 & 0.4671 & 0.4255 & 0.4 & & 0.683 \\
\hline B & 0.46 & 0 & 1.5 & & & 1.413 \\
\hline$B$ & 0.4255 & 29 & 0 & 1.5 & 0. & 0.109 \\
\hline$B$ & 0.4671 & 90 & 1.5029 & 0.0 & 1.4131 & 0.1163 \\
\hline $\mathrm{Si}$ & 6834 & 0.1163 & 0.1093 & 1.41 & 0 & 43 \\
\hline $8 \mathrm{Si}$ & 6834 & 1.4131 & 1093 & 0.1163 & 0.0543 & 0.000 \\
\hline
\end{tabular}

24. $\mathrm{C}_{2 \mathrm{v}} \mathrm{B}_{3} \mathrm{Si}_{2} \mathrm{P}$

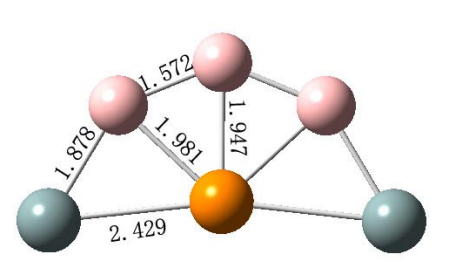

\begin{tabular}{cccccc} 
& $\mathrm{X} / \AA$ & $\mathrm{Y} / \AA$ & \multicolumn{1}{c}{$\mathrm{Z} / \AA$} & $\mathrm{q} / \mathrm{e}$ & WBI \\
$\mathrm{P}$ & 0.000000 & 0.000000 & 0.271264 & +0.25 & 3.53 \\
$\mathrm{~B}$ & 0.000000 & 1.453311 & -1.075041 & -0.63 & 3.73 \\
$\mathrm{~B}$ & 0.000000 & 0.000000 & -1.675250 & 0.0061 & 3.42 \\
$\mathrm{~B}$ & 0.000000 & -1.453311 & -1.075041 & -0.63 & 3.73 \\
$\mathrm{Si}$ & 0.000000 & -2.414720 & 0.537775 & +0.51 & 2.30 \\
$\mathrm{Si}$ & 0.000000 & 2.414720 & 0.537775 & +0.51 & 2.30
\end{tabular}

Wiberg bond Index matrix

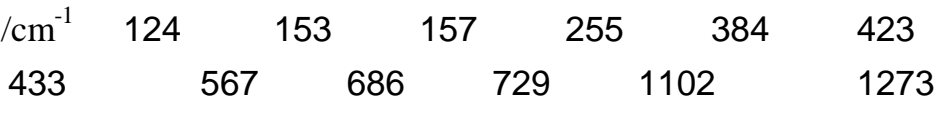

$\begin{array}{lccccccc}\text { Atom } & 1 & 2 & 3 & 4 & 5 & 6 \\ \text { 1. } & \text { P } & 0.0000 & 0.8774 & 0.8387 & 0.8774 & 0.4699 & 0.4699 \\ \text { 2. } & \text { B } & 0.8774 & 0.0000 & 1.2281 & 0.0624 & 0.2083 & 1.3568 \\ \text { 3. } & \text { B } & 0.8387 & 1.2281 & 0.0000 & 1.2281 & 0.0626 & 0.0626 \\ \text { 4. } & \text { B } & 0.8774 & 0.0624 & 1.2281 & 0.0000 & 1.3568 & 0.2083 \\ \text { 5. Si } & 0.4699 & 0.2083 & 0.0626 & 1.3568 & 0.0000 & 0.2000 \\ \text { 6. Si } & 0.4699 & 1.3568 & 0.0626 & 0.2083 & 0.2000 & 0.0000\end{array}$




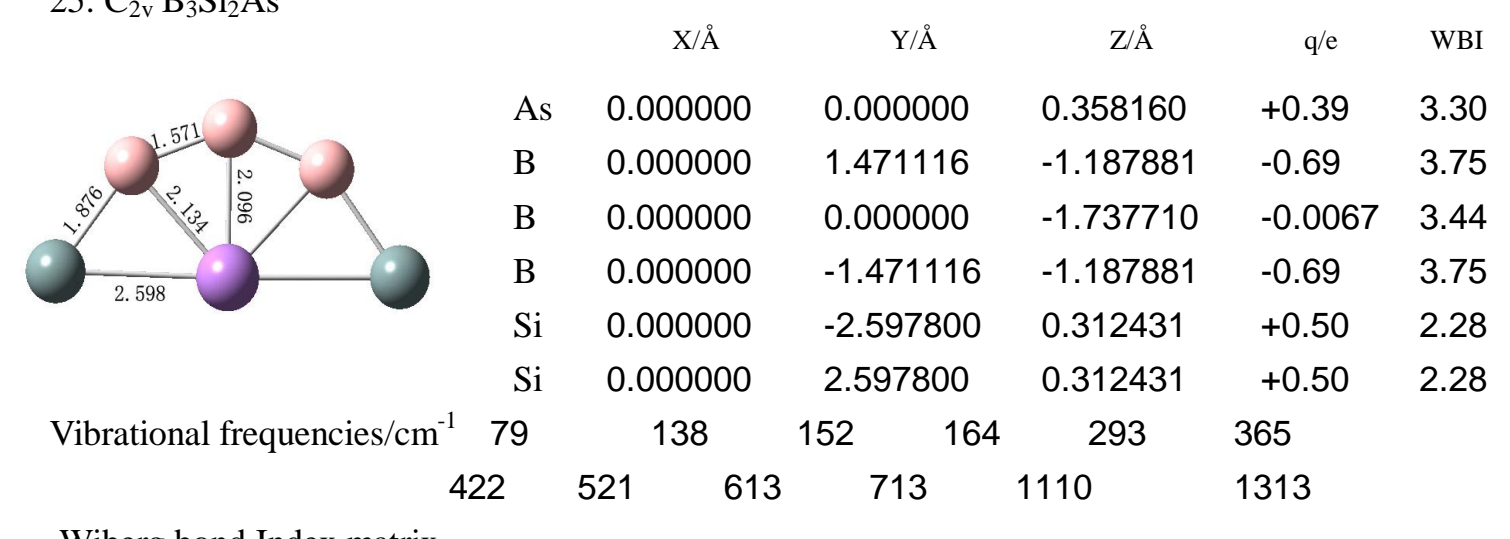

Wiberg bond Index matrix

\begin{tabular}{|c|c|c|c|c|c|c|}
\hline \multirow[t]{2}{*}{ Atom } & 1 & 2 & 3 & 4 & 5 & 6 \\
\hline & ---- & 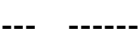 & ------ & ----- & & \\
\hline 1. As & 0.0000 & 0.8420 & 0.7926 & 0.8420 & 0.4135 & 0.4135 \\
\hline 2. $B$ & 0.8420 & 0.0000 & 1.2618 & 0.0503 & 0.1923 & 1.4037 \\
\hline 3. $\mathrm{B}$ & 0.7926 & 1.2618 & 0.0000 & 1.2618 & 0.0595 & 0.0595 \\
\hline 4. $\mathrm{B}$ & 0.8420 & 0.0503 & 1.2618 & 0.0000 & 1.4037 & 0.1923 \\
\hline 5. $\varsigma$ & 0.4135 & 0.1923 & 0.0595 & 1.4037 & 0.0000 & 0.2136 \\
\hline 6. S & 0.4135 & 1.4037 & 0.0595 & 0.1923 & 0.2136 & 0.0000 \\
\hline
\end{tabular}

26. $\mathrm{C}_{2 \mathrm{v}} \mathrm{B}_{5} \mathrm{H}_{2} \mathrm{Si}^{-}$

$\begin{array}{ccccccc} & & \mathrm{X} / \AA & \mathrm{Y} / \AA & \mathrm{Z} / \AA & \mathrm{q} / \mathrm{e} & \mathrm{WBI} \\ & \mathrm{Si} & 0.000000 & 0.000000 & 0.581707 & +0.80 & 3.41 \\ \mathrm{H} & 0.000000 & 2.835549 & 1.410329 & -0.059 & 1.00 \\ \mathrm{~B} & 0.000000 & 2.072051 & 0.507080 & -0.35 & 3.84 \\ \mathrm{~B} & 0.000000 & 1.447099 & -0.874028 & -0.43 & 3.83 \\ \mathrm{~B} & 0.000000 & 0.000000 & -1.459014 & -0.12 & 3.57 \\ \mathrm{~B} & 0.000000 & -1.447099 & -0.874028 & -0.43 & 3.83 \\ \mathrm{~B} & 0.000000 & -2.072051 & 0.507080 & -0.35 & 3.84 \\ \mathrm{H} & 0.000000 & -2.835549 & 1.410329 & -0.059 & 1.00\end{array}$

\begin{tabular}{rlllllll} 
Vibrational frequencies/cm & \multicolumn{1}{c}{175} & \multicolumn{2}{c}{225} & \multicolumn{2}{c}{310} & 454 & \multicolumn{2}{l}{467} & 482 \\
511 & 619 & 627 & 661 & 718 & 746 \\
817 & 1053 & 1318 & 1370 & 2685 & 2687
\end{tabular}

Wiberg bond Index matrix

\begin{tabular}{|c|c|c|c|c|c|c|c|c|}
\hline Atom & 1 & 2 & 3 & 4 & 5 & 6 & 7 & 3 \\
\hline & & ---- & . & - - - & --- & ----- & & \\
\hline 1. $\mathrm{Si}$ & 0.0000 & 0.0079 & 0.7826 & 0.5878 & 0.6557 & 0.5878 & 0.7826 & 0.0079 \\
\hline 2. $\mathrm{H}$ & 0.0079 & 0.0000 & 0.9748 & 0.0084 & 0.0072 & 0.0045 & 0.0003 & 0.0002 \\
\hline 3. $B$ & 0.7826 & 0.9748 & 0.0000 & 1.7110 & 0.0895 & 0.1245 & 0.1570 & 0.0003 \\
\hline 4. $\mathrm{B}$ & 0.5878 & 0.0084 & 1.7110 & 0.0000 & 1.3608 & 0.0309 & 0.1245 & 0.0045 \\
\hline
\end{tabular}



$\begin{array}{llllllllll}\text { 5. B } & 0.6557 & 0.0072 & 0.0895 & 1.3608 & 0.0000 & 1.3608 & 0.0895 & 0.0072\end{array}$
6. B $\begin{array}{llllllllll}0.5878 & 0.0045 & 0.1245 & 0.0309 & 1.3608 & 0.0000 & 1.7110 & 0.0084\end{array}$

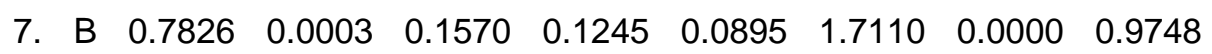
8. H $\begin{array}{lllllllll}0.0079 & 0.0002 & 0.0003 & 0.0045 & 0.0072 & 0.0084 & 0.9748 & 0.0000\end{array}$ 27. $\mathrm{C}_{2 \mathrm{v}} \mathrm{B}_{3} \mathrm{C}_{2} \mathrm{H}_{2} \mathrm{Si}^{+}$

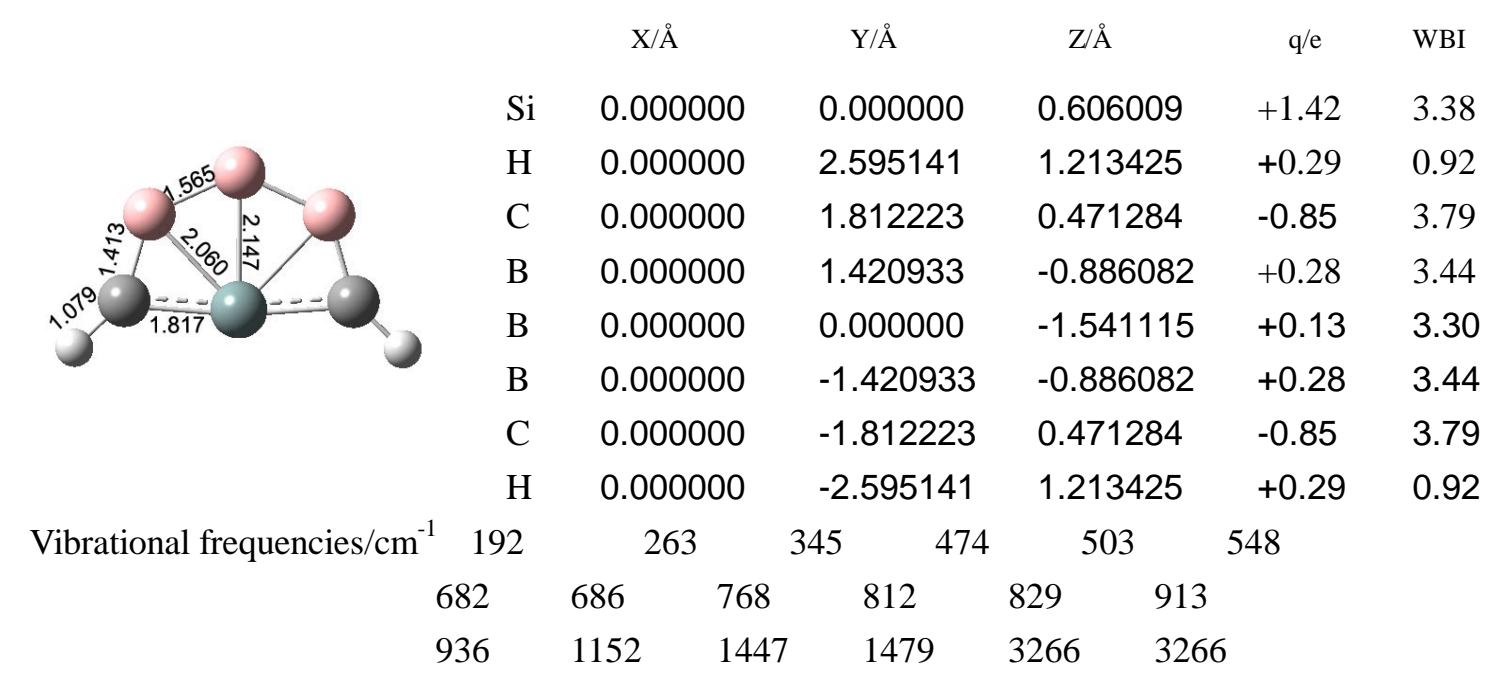

Wiberg bond Index matrix

\begin{tabular}{|c|c|c|c|c|c|c|c|c|}
\hline$n$ & 1 & 2 & 3 & & & & & \\
\hline & & & & 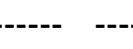 & & ----- & & \\
\hline II & 0.0000 & 0.0 & 1.0160 & 0.3982 & 0.5 & 0.3982 & 60 & $v$ \\
\hline $\mathrm{H}$ & 0.0 & 00 & 2 & & & 0.0 & & 00 \\
\hline C & 1.0 & 2 & & & & & & U \\
\hline B & 3 & 4 & & & & & & 08 \\
\hline B & 0.5 & 6 & 1 & 1. & 0 & 1.2 & 0 & 116 \\
\hline$B$ & 0.3982 & 6 & 1 & 3 & 9 & 0 & 6 & 08 \\
\hline C & - & 3 & & 1 & 31 & 26 & 00 & 380 \\
\hline $\mathrm{H}$ & 0.0092 & 0.0005 & 0.0003 & 0.0086 & 0.0116 & 0.0084 & 0.8802 & 0.000 \\
\hline
\end{tabular}

28. $\mathrm{C}_{\mathrm{s}} \mathrm{B}_{4} \mathrm{C} \mathrm{H}_{2} \mathrm{Si}$

$\begin{array}{ccccccc} & & \mathrm{X} / \AA & \mathrm{Y} / \AA & \mathrm{Z} / \AA & \mathrm{q} / \mathrm{e} & \text { WBI } \\ & \mathrm{Si} & 0.000000 & 0.595413 & 0.000000 & +1.06 & 3.34 \\ \mathrm{H} & 2.773028 & 1.508468 & 0.000000 & -0.036 & 1.01 \\ & \mathrm{~B} & 2.040405 & 0.589878 & 0.000000 & -0.12 & 3.67 \\ & \mathrm{~B} & 1.466300 & -0.834520 & 0.000000 & -0.34 & 3.77 \\ & \mathrm{~B} & 0.085734 & -1.488222 & 0.000000 & -0.062 & 3.54 \\ & \mathrm{~B} & -1.387305 & -0.924124 & 0.000000 & +0.21 & 3.50 \\ & \mathrm{C} & -1.859526 & 0.386299 & 0.000000 & -0.96 & 3.76 \\ & \mathrm{H} & -2.641538 & 1.122900 & 0.000000 & +0.25 & 0.94\end{array}$


\begin{tabular}{rllllllll} 
Vibrational frequencies/cm & 166 & \multicolumn{2}{c}{253} & \multicolumn{2}{c}{340} & 447 & 463 & 532 \\
616 & 623 & 661 & 702 & 733 & 835 \\
886 & 1092 & 1381 & 1501 & 2762 & 3300
\end{tabular}

Wiberg bond Index matrix

$\begin{array}{lllrrrrrr}\text { Atom } & 1 & 2 & 3 & 4 & 5 & 6 & 7 & 8 \\ - & \end{array}$

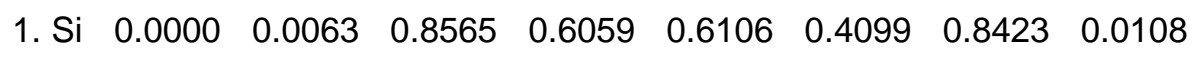

2. $\begin{array}{lllllllll}\mathrm{H} & 0.0063 & 0.0000 & 0.9801 & 0.0073 & 0.0062 & 0.0062 & 0.0003 & 0.0004\end{array}$

3. B $\begin{array}{lllllllll}0.8565 & 0.9801 & 0.0000 & 1.4984 & 0.1538 & 0.0522 & 0.1255 & 0.0004\end{array}$

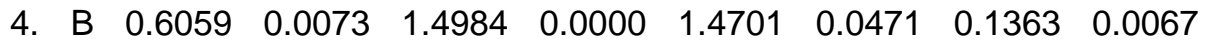

$\begin{array}{llllllllll}\text { 5. } & \text { B } & 0.6106 & 0.0062 & 0.1538 & 1.4701 & 0.0000 & 1.2531 & 0.0316 & 0.0119\end{array}$

6. B $\begin{array}{lllllllll}0.4099 & 0.0062 & 0.0522 & 0.0471 & 1.2531 & 0.0000 & 1.7265 & 0.0097\end{array}$

7. $\begin{array}{lllllllll}\text { C } & 0.8423 & 0.0003 & 0.1255 & 0.1363 & 0.0316 & 1.7265 & 0.0000 & 0.9006\end{array}$

8. H $\begin{array}{lllllllll}\text { H } & 0.0108 & 0.0004 & 0.0004 & 0.0067 & 0.0119 & 0.0097 & 0.9006 & 0.0000\end{array}$

29. $\mathrm{C}_{2 \mathrm{v}} \mathrm{B}_{4} \mathrm{Si}_{2} \mathrm{C}$

\begin{tabular}{|c|c|c|c|c|c|c|}
\hline & & X/Å & Y/Å & Z/Å & $q / e$ & WBI \\
\hline & $\mathrm{C}$ & 0.000000 & 0.000000 & 0.576757 & -0.90 & 3.77 \\
\hline & $\mathrm{Si}$ & 0.000000 & 1.561032 & -1.104404 & +0.75 & 2.08 \\
\hline & B & 0.000000 & 1.646119 & 0.744815 & -0.53 & 3.65 \\
\hline & B & 0.000000 & 0.788407 & 2.001462 & +0.23 & 3.26 \\
\hline & B & 0.000000 & -0.788407 & 2.001462 & +0.23 & 3.26 \\
\hline & B & 0.000000 & -1.646119 & 0.744815 & -0.53 & 3.65 \\
\hline & $\mathrm{Si}$ & 0.000000 & -1.561032 & -1.104404 & +0.75 & 2.08 \\
\hline Vibrational frequencies $/ \mathrm{cm}^{-1}$ & -138 & 63 & 156 & 161 & 401 & \\
\hline & 434 & 578 & 586 & 629 & 836 & \\
\hline & 1037 & 1295 & 1397 & & & \\
\hline
\end{tabular}

Wiberg bond Index matrix

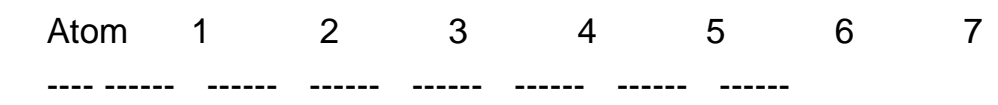

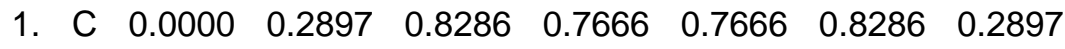

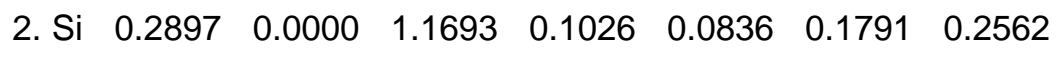

3. B $\begin{array}{llllllll}0.8286 & 1.1693 & 0.0000 & 1.2324 & 0.0263 & 0.2128 & 0.1791\end{array}$

4. $\begin{array}{lllllllll}\text { B } & 0.7666 & 0.1026 & 1.2324 & 0.0000 & 1.0529 & 0.0263 & 0.0836\end{array}$

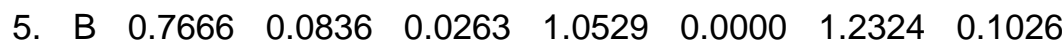

6. B $\begin{array}{lllllllll}0.8286 & 0.1791 & 0.2128 & 0.0263 & 1.2324 & 0.0000 & 1.1693\end{array}$

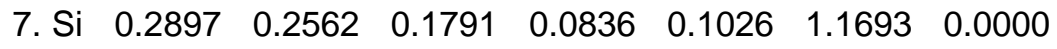


30. $\mathrm{C}_{2 \mathrm{v}} \mathrm{B}_{4} \mathrm{Si}_{2} \mathrm{C}^{-}$

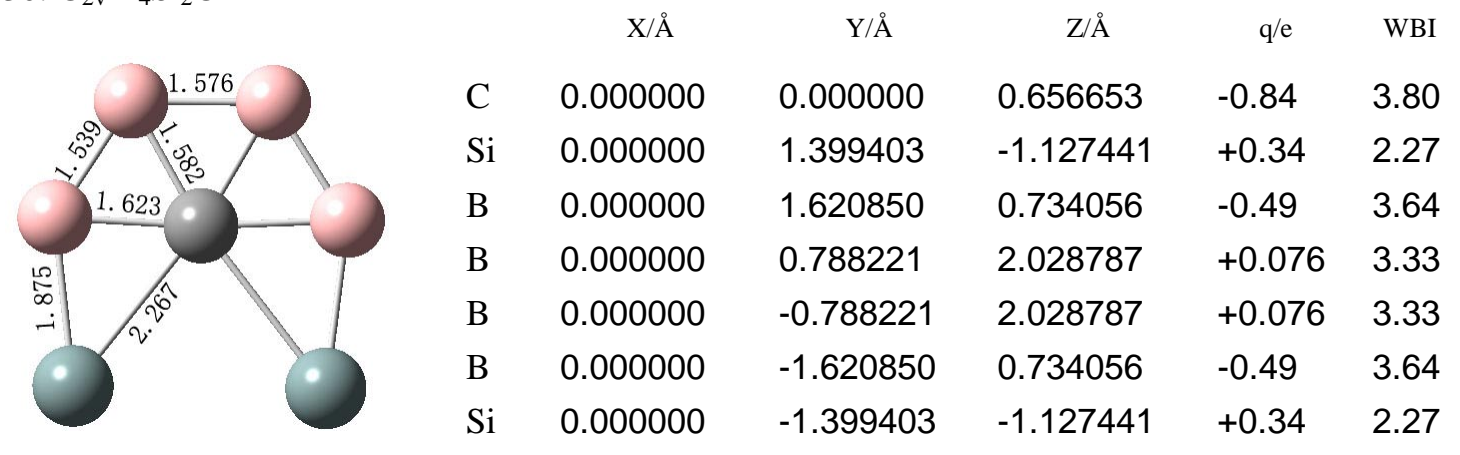

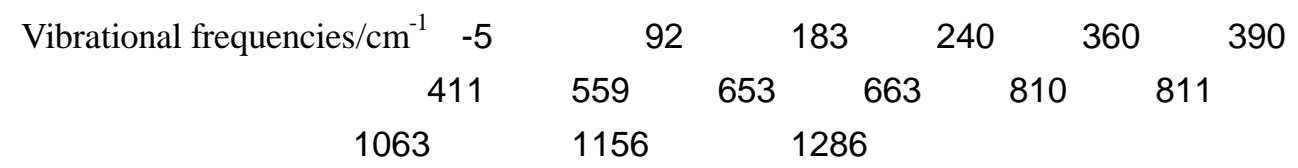

Wiberg bond Index matrix

$\begin{array}{lrrrrrrr}\text { Atom } & 1 & 2 & 3 & 4 & 5 & 6 & 7\end{array}$

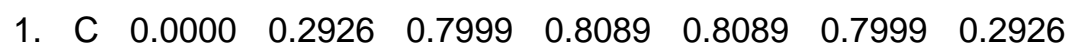

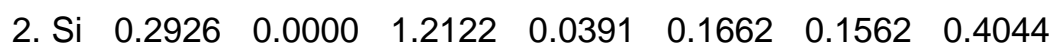

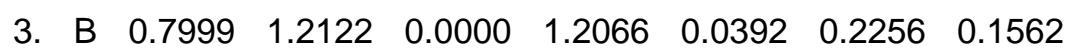

$\begin{array}{llllllllll}\text { 4. } & \text { B } & 0.8089 & 0.0391 & 1.2066 & 0.0000 & 1.0683 & 0.0392 & 0.1662\end{array}$

$\begin{array}{llllllllll}\text { 5. } & \text { B } & 0.8089 & 0.1662 & 0.0392 & 1.0683 & 0.0000 & 1.2066 & 0.0391\end{array}$

6. B $\quad \begin{array}{llllllll}\text { B } & 0.7999 & 0.1562 & 0.2256 & 0.0392 & 1.2066 & 0.0000 & 1.2122\end{array}$

$\begin{array}{llllllll}\text { 7. Si } & 0.2926 & 0.4044 & 0.1562 & 0.1662 & 0.0391 & 1.2122 & 0.0000\end{array}$

31. $\mathrm{C}_{2 \mathrm{v}} \mathrm{B}_{4} \mathrm{Si}_{2} \mathrm{Si}$

\begin{tabular}{|c|c|c|c|c|c|}
\hline & $\mathrm{X} / \AA$ & Y/Å & Z/Å & $\mathrm{q} / \mathrm{e}$ & WBI \\
\hline $\mathrm{Si}$ & 0.000000 & 0.000000 & 0.030468 & +0.53 & 3.60 \\
\hline $\mathrm{Si}$ & 0.000000 & 2.308113 & 0.974320 & +0.55 & 2.34 \\
\hline B & 0.000000 & 1.901489 & -0.854549 & -0.74 & 3.80 \\
\hline B & 0.000000 & 0.782077 & -1.916203 & -0.073 & 3.56 \\
\hline B & 0.000000 & -0.782077 & -1.916203 & -0.073 & 3.56 \\
\hline B & 0.000000 & -1.901489 & -0.854549 & -0.74 & 3.80 \\
\hline $\mathrm{Si}$ & 0.000000 & -2.308113 & 0.974320 & +0.55 & 2.34 \\
\hline
\end{tabular}

\begin{tabular}{llllrrr} 
Vibrational frequencies/cm & -1 & 121 & 159 & 224 & 261 & 360 \\
& 412 & 446 & 484 & 566 & 635 & 680 \\
& 977 & 1229 & \multicolumn{2}{c}{1420} & &
\end{tabular}

Wiberg bond Index matrix

$\begin{array}{cccccccc}\text { Atom } & 1 & 2 & 3 & 4 & 5 & 6 & 7 \\ - & -1 & -1 & & \end{array}$




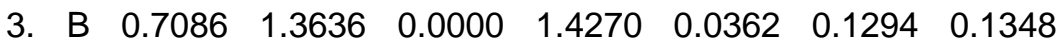

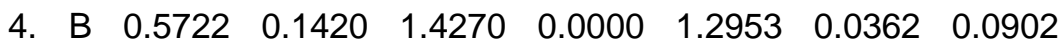

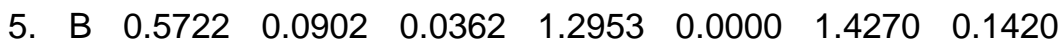

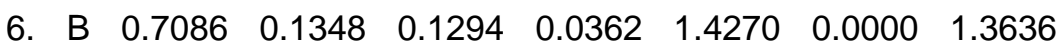

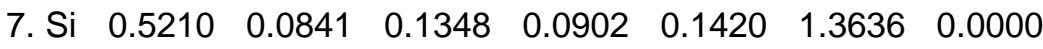

32. $\mathrm{C}_{2 \mathrm{v}} \mathrm{B}_{4} \mathrm{Si}_{2} \mathrm{Si}^{-}$

$\begin{array}{llllll}\mathrm{Si} & 0.000000 & 0.000000 & 0.026096 & +0.57 & 3.65 \\ \mathrm{Si} & 0.000000 & 2.214425 & 0.990963 & +0.17 & 2.49 \\ \mathrm{~B} & 0.000000 & 1.878502 & -0.855954 & -0.70 & 3.83 \\ \mathrm{~B} & 0.000000 & 0.767778 & -1.955278 & -0.26 & 3.49 \\ \mathrm{~B} & 0.000000 & -0.767778 & -1.955278 & -0.26 & 3.49 \\ \mathrm{~B} & 0.000000 & -1.878502 & -0.855954 & -0.70 & 3.83 \\ \mathrm{Si} & 0.000000 & -2.214425 & 0.990963 & +0.17 & 2.49\end{array}$

$\begin{array}{lllllll}\text { Vibrational frequencies } / \mathrm{cm}^{-1} & 138 & 154 & 171 & 290 & 291 & 321\end{array}$

$\begin{array}{lllll}979 & 1145 & 1381 & 436 & \end{array}$

Wiberg bond Index matrix

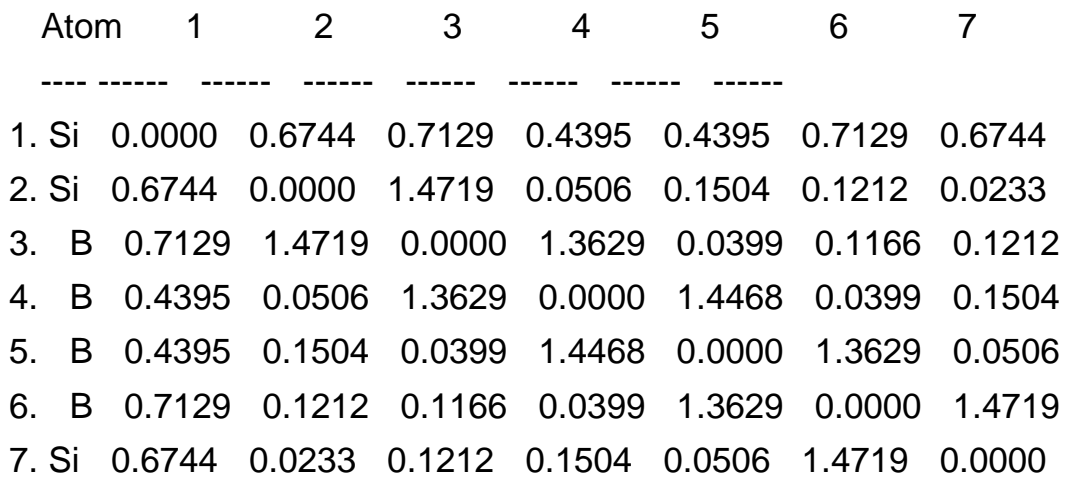

33. $\mathrm{C}_{2 \mathrm{v}} \mathrm{B}_{4} \mathrm{Si}_{2} \mathrm{Ge}$

\begin{tabular}{|c|c|c|c|c|c|}
\hline & $\mathrm{X} / \AA ̊ \AA$ & Y/Å & Z/Å & $q / e$ & WBI \\
\hline $\mathrm{Ge}$ & 0.000000 & 0.000000 & 0.135129 & +0.50 & 3.36 \\
\hline $\mathrm{Si}$ & 0.000000 & 2.478178 & 0.872275 & +0.54 & 2.33 \\
\hline B & 0.000000 & 1.943122 & -0.929378 & -0.73 & 3.7 \\
\hline B & 0.000000 & 0.782403 & -1.945406 & -0.065 & 0.6 \\
\hline B & 0.000000 & -0.782403 & -1.945406 & -0.065 & 3.5 \\
\hline B & 0.000000 & -1.943122 & -0.929378 & -0.73 & 3.7 \\
\hline $\mathrm{Si}$ & 0.000000 & -2.478178 & 0.872275 & +0.54 & 2.3 \\
\hline
\end{tabular}




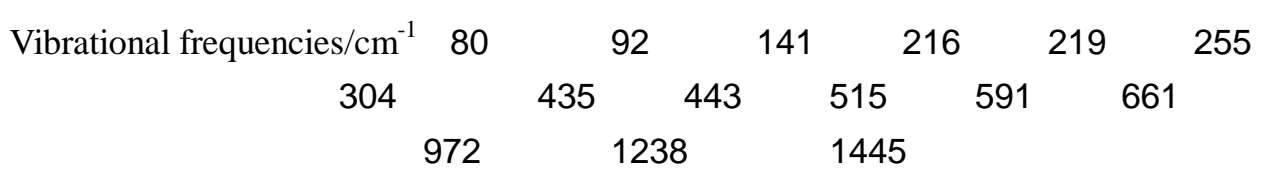

Wiberg bond Index matrix

\begin{tabular}{|c|c|c|c|c|c|c|c|}
\hline & \multicolumn{5}{|c|}{$\begin{array}{lllllll}\text { Atom } & 1 & 2 & 3 & 4 & 5 & 6\end{array}$} \\
\hline \multicolumn{8}{|c|}{---- ------ $\quad$------ $\quad$------ $\quad$------ $\quad$------ $\quad$------ $\quad$------ } \\
\hline \multicolumn{8}{|c|}{$\begin{array}{llllllll}. \mathrm{Ge} & 0.0000 & 0.4885 & 0.6506 & 0.5408 & 0.5408 & 0.6506 & 0.4885\end{array}$} \\
\hline \multicolumn{8}{|c|}{$\begin{array}{lllllll}0.4885 & 0.0000 & 1.3774 & 0.1475 & 0.0799 & 0.1482 & 0.0835\end{array}$} \\
\hline \\
\hline \\
\hline \multicolumn{8}{|l|}{5.} \\
\hline \multirow[t]{2}{*}{ 6. B } & 0.6506 & 0.1482 & 0.1 & 0.0 & 1.4413 & 0.0000 & 1.3774 \\
\hline & 0.4885 & 0.0835 & 0.1482 & 0.0799 & 0.1475 & 1.3774 & 0.0000 \\
\hline
\end{tabular}

34. $\mathrm{C}_{2 \mathrm{v}} \mathrm{B}_{4} \mathrm{Si}_{2} \mathrm{Ge}^{-}$

$\begin{array}{lcccccr} & & \mathrm{X} / \AA & \mathrm{Y} / \AA & \mathrm{Z} / \AA & \mathrm{q} / \mathrm{e} & \text { WBI } \\ \mathrm{Ge} & 0.000000 & 0.000000 & 0.148722 & +0.50 & 3.44 \\ \mathrm{Si} & 0.000000 & 2.381561 & 0.882689 & +0.16 & 2.51 \\ \mathrm{~B} & 0.000000 & 1.906047 & -0.939057 & -0.68 & 3.80 \\ \mathrm{~B} & 0.000000 & 0.767652 & -2.008382 & -0.23 & 3.44 \\ \mathrm{~B} & 0.000000 & -0.767652 & -2.008382 & -0.23 & 3.44 \\ \mathrm{~B} & 0.000000 & -1.906047 & -0.939057 & -0.68 & 3.80 \\ \mathrm{Si} & 0.000000 & -2.381561 & 0.882689 & +0.16 & 2.51\end{array}$

\begin{tabular}{llllrrr} 
Vibrational frequencies $/ \mathrm{cm}^{-1}$ & 95 & 145 & 151 & 209 & 251 & 288 \\
& 318 & 391 & 449 & 512 & 583 & 646 \\
& 980 & 1153 & \multicolumn{2}{c}{1409} & &
\end{tabular}

Wiberg bond Index matrix

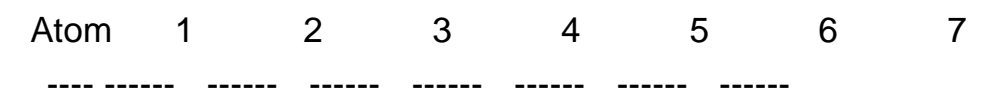

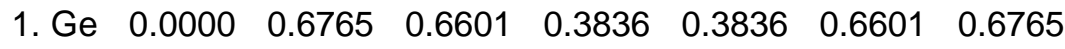

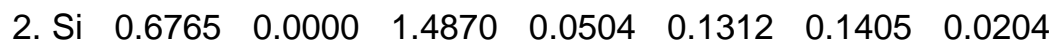

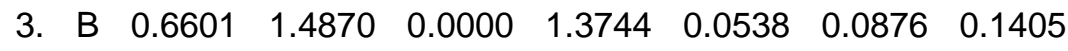

4. B $\begin{array}{llllllll}0.3836 & 0.0504 & 1.3744 & 0.0000 & 1.4492 & 0.0538 & 0.1312\end{array}$

$\begin{array}{lllllllll}\text { 5. } & \text { B } & 0.3836 & 0.1312 & 0.0538 & 1.4492 & 0.0000 & 1.3744 & 0.0504\end{array}$

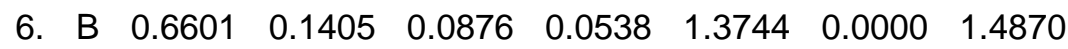

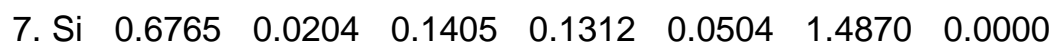


35. $\mathrm{C}_{2 \mathrm{v}} \mathrm{B}_{4} \mathrm{Si}_{2} \mathrm{~B}^{-}$

\begin{tabular}{llccccc} 
& & $\mathrm{X} / \AA$ & $\mathrm{Y} / \AA$ & \multicolumn{1}{c}{$\mathrm{Z} / \AA$} & $\mathrm{q} / \mathrm{e}$ & WBI \\
$\mathrm{B}$ & 0.000000 & 0.000000 & 0.416980 & -0.41 & 3.83 \\
$\mathrm{Si}$ & 0.000000 & 1.670242 & -1.057771 & +0.46 & 2.39 \\
$\mathrm{~B}$ & 0.000000 & 1.749246 & 0.785162 & -0.70 & 3.84 \\
$\mathrm{~B}$ & 0.000000 & 0.783218 & 1.968106 & -0.052 & 3.54 \\
$\mathrm{~B}$ & 0.000000 & -0.783218 & 1.968106 & -0.052 & 3.54 \\
$\mathrm{~B}$ & 0.000000 & -1.749246 & 0.785162 & -0.70 & 3.84 \\
$\mathrm{Si}$ & 0.000000 & -1.670242 & -1.057771 & +0.46 & 2.39
\end{tabular}

\begin{tabular}{rrlrrrr} 
Vibrational frequencies $/ \mathrm{cm}^{-1}-85$ & 166 & 177 & 255 & 376 & 426 \\
426 & 599 & 617 & 630 & 780 & 808 \\
1009 & 1265 & \multicolumn{2}{c}{1387} & &
\end{tabular}

Wiberg bond Index matrix

\begin{tabular}{|c|c|c|c|c|}
\hline Atom & 1 & 2 & 3 & \\
\hline
\end{tabular}

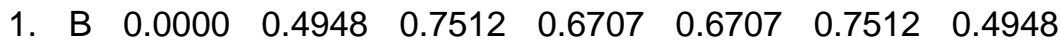

$\begin{array}{llllllll}\text { 2. Si } & 0.4948 & 0.0000 & 1.3727 & 0.1449 & 0.0924 & 0.1297 & 0.1514\end{array}$

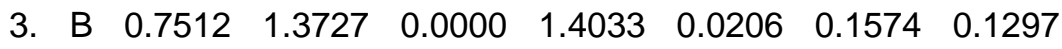

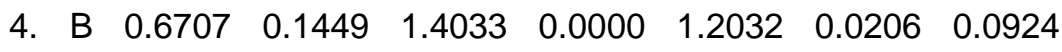

$\begin{array}{llllllllll}\text { 5. B } & 0.6707 & 0.0924 & 0.0206 & 1.2032 & 0.0000 & 1.4033 & 0.1449\end{array}$

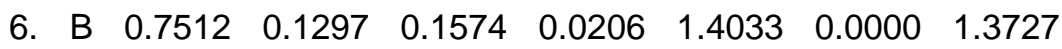

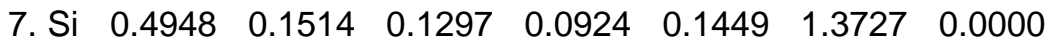

36. $\mathrm{C}_{2 \mathrm{v}} \mathrm{B}_{4} \mathrm{Si}_{2} \mathrm{Al}^{-}$

$\begin{array}{ccccccc} & & \mathrm{X} / \AA & \mathrm{Y} / \AA & \mathrm{Z} / \AA & \mathrm{q} / \mathrm{e} & \mathrm{WBI} \\ \mathrm{Al} & 0.000000 & 0.000000 & 0.200693 & +0.86 & 2.82 \\ \mathrm{Si} & 0.000000 & 2.472591 & 0.901029 & +0.16 & 2.73 \\ \mathrm{~B} & 0.000000 & 1.933836 & -0.880149 & -0.86 & 3.91 \\ \mathrm{~B} & 0.000000 & 0.776150 & -1.903633 & -0.22 & 3.67 \\ \mathrm{~B} & 0.000000 & -0.776150 & -1.903633 & -0.22 & 3.67 \\ \mathrm{~B} & 0.000000 & -1.933836 & -0.880149 & -0.86 & 3.91 \\ \mathrm{Si} & 0.000000 & -2.472591 & 0.901029 & +0.16 & 2.73\end{array}$

\begin{tabular}{ccccccc} 
Vibrational frequencies/cm & 97 & \multicolumn{1}{c}{116} & 166 & 238 & 272 & 324 \\
& 406 & 457 & 472 & 539 & 595 & 673 \\
975 & 1216 & 1428 & & &
\end{tabular} 
Wiberg bond Index matrix

\begin{tabular}{lllllllll}
\multicolumn{2}{l}{ Atom } & 1 & 2 & 3 & 4 & 5 & 6 & 7 \\
1. Al & 0.0000 & 0.5953 & 0.4870 & 0.3288 & 0.3288 & 0.4870 & 0.5953 \\
2. Si & 0.5953 & 0.0000 & 1.6356 & 0.1758 & 0.1062 & 0.1173 & 0.1009 \\
3. & B & 0.4870 & 1.6356 & 0.0000 & 1.5329 & 0.0743 & 0.0660 & 0.1173 \\
4. & B & 0.3288 & 0.1758 & 1.5329 & 0.0000 & 1.4491 & 0.0743 & 0.1062 \\
5. & B & 0.3288 & 0.1062 & 0.0743 & 1.4491 & 0.0000 & 1.5329 & 0.1758 \\
6. & B & 0.4870 & 0.1173 & 0.0660 & 0.0743 & 1.5329 & 0.0000 & 1.6356 \\
7. Si & 0.5953 & 0.1009 & 0.1173 & 0.1062 & 0.1758 & 1.6356 & 0.0000
\end{tabular}

37. $\mathrm{C}_{2 \mathrm{v}} \mathrm{B}_{4} \mathrm{Si}_{2} \mathrm{Ga}^{-}$

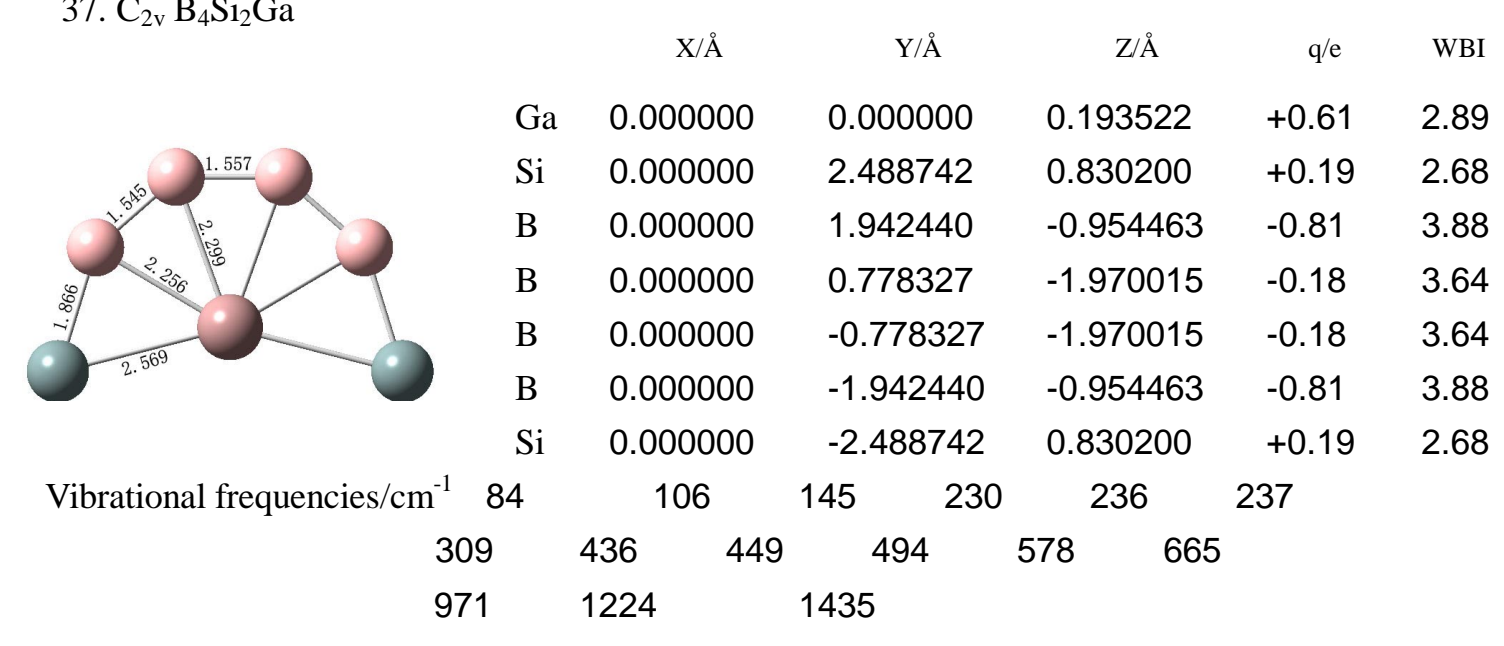

Wiberg bond Index matrix

\begin{tabular}{|c|c|c|c|c|c|c|c|}
\hline \multicolumn{2}{|c|}{ Atom } & 2 & 3 & 4 & 5 & 6 & 7 \\
\hline & - & & 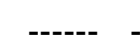 & --- & ----- & & \\
\hline $\mathrm{Ga}$ & 0.0000 & 0.5924 & 0.4990 & 0.3542 & 0.3542 & 90 & 0.5924 \\
\hline $\mathrm{Si}$ & 0.5924 & 0.0000 & 914 & 0.17 & 0.1004 & 0.1301 & 0.0889 \\
\hline B & 0.4990 & 14 & 000 & 1.5214 & 0.0730 & 51 & 0. \\
\hline$B$ & 3542 & 95 & 4 & 0.0 & 1.4 & 30 & 04 \\
\hline$B$ & 35 & 04 & 730 & 1.4097 & 0.0000 & 1.5214 & 0.1795 \\
\hline & . & 1 & 1 & 0.0 & 1.5214 & 000 & 1. \\
\hline $\mathrm{Si}$ & .5924 & 0889 & 130 & 00 & .1795 & 1.5914 & 0.0000 \\
\hline
\end{tabular}

38. $\mathrm{C}_{2 \mathrm{v}} \mathrm{B}_{4} \mathrm{Si}_{2} \mathrm{P}$

$\begin{array}{ccccccc} & & \mathrm{X} / \AA & \mathrm{Y} / \AA & \mathrm{Z} / \AA & \mathrm{q} / \mathrm{e} & \text { WBI } \\ \mathrm{P} & 0.000000 & 0.000000 & 0.004825 & +0.17 & 3.67 \\ \mathrm{Si} & 0.000000 & 2.101425 & -1.019278 & +0.55 & 2.24 \\ \mathrm{~B} & 0.000000 & 1.834490 & 0.851874 & -0.58 & 3.68 \\ \mathrm{~B} & 0.000000 & 0.770912 & 1.994868 & -0.058 & 3.26 \\ \mathrm{~B} & 0.000000 & -0.770912 & 1.994868 & -0.058 & 3.26 \\ \mathrm{~B} & 0.000000 & -1.834490 & 0.851874 & -0.58 & 3.68 \\ \mathrm{Si} & 0.000000 & -2.101425 & -1.019278 & +0.55 & 2.24\end{array}$


\begin{tabular}{rrrrrrrrr} 
Vibrational frequencies/cm & 111 & \multicolumn{1}{c}{137} & 165 & 271 & 276 & 281 \\
374 & & 374 & 443 & 513 & 614 & 669
\end{tabular}

Wiberg bond Index matrix

\begin{tabular}{|c|c|c|c|c|c|c|c|}
\hline & \multicolumn{6}{|c|}{ Atom 1} \\
\hline \multicolumn{8}{|c|}{---- ------ ------ ----- } \\
\hline \multicolumn{2}{|c|}{ P 0.0000} & 0.5997 & $\begin{array}{l}-1.7839 \\
0.0\end{array}$ & 0.4489 & 0.4489 & 0.7839 & 0.5997 \\
\hline 2. $\mathrm{Si}$ & 0.5997 & 0.0000 & 1.2536 & 0.0463 & 0.1356 & 0.1632 & 0.0414 \\
\hline \multicolumn{2}{|c|}{ В 0.7839} & 1.2536 & 0.0000 & 1.2 & 0.0 & 17 & 0.1632 \\
\hline \multicolumn{2}{|c|}{ В 0.4489} & 0.0463 & 1.2808 & 0.0000 & 1.3149 & 0.0290 & 0.1356 \\
\hline \multicolumn{2}{|c|}{ В 0.4489} & 0. & 0.0290 & 1.3 & 0.0000 & 1.2808 & 0.0463 \\
\hline \multirow[t]{2}{*}{ 6. $B$} & 0.7839 & 0.1632 & 0.1 & 0 & 1.2808 & 00 & 36 \\
\hline & 0.5997 & 0.0414 & 0.1632 & 0.1356 & 0.0463 & 1.2536 & 0.0000 \\
\hline
\end{tabular}

39. $\mathrm{C}_{2 \mathrm{v}} \mathrm{B}_{4} \mathrm{Si}_{2} \mathrm{As}$

$\begin{array}{ccccccr} & & \text { X/A } & \text { Y/A } & \text { Z/A } & \text { q/e } & \text { WBI } \\ \text { As } & 0.000000 & 0.000000 & 0.165280 & +0.26 & 3.41 \\ \text { Si } & 0.000000 & 2.346009 & 0.880722 & +0.52 & 2.31 \\ \text { B } & 0.000000 & 1.877847 & -0.957039 & -0.59 & 3.67 \\ \text { B } & 0.000000 & 0.771005 & -2.054408 & -0.054 & 3.24 \\ \text { B } & 0.000000 & -0.771005 & -2.054408 & -0.054 & 3.24 \\ \text { B } & 0.000000 & -1.877847 & -0.957039 & -0.59 & 3.67 \\ \text { Si } & 0.000000 & -2.346009 & 0.880722 & +0.52 & 2.31\end{array}$

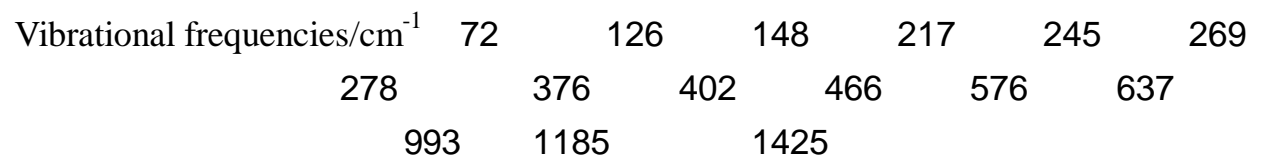

Wiberg bond Index matrix

\begin{tabular}{|c|c|c|c|c|c|c|c|}
\hline \multirow{2}{*}{\multicolumn{8}{|c|}{ Atom 1}} \\
\hline & & & & & & & \\
\hline As & 0.0000 & 0.6158 & 0.7114 & 0.3780 & 0.3780 & 114 & 0.6158 \\
\hline $\mathrm{Si}$ & 0.6158 & 0.0000 & 1.2976 & 0.0527 & 0.1044 & 0.1780 & 0.0607 \\
\hline 3. B & 0.7114 & 1.2976 & 0.0000 & 1.3227 & 0.0475 & 0.1163 & 0.1780 \\
\hline 1. B & 0.3780 & 0.0527 & 1.3227 & 0.0000 & 1.3302 & 0.0475 & 0.1044 \\
\hline 5. B & 0.37 & 0.1 & 0.0475 & 1.3 & 0.0000 & 1.3227 & 0.0527 \\
\hline$B$ & 0.71 & 30 & 0 . & 0.0 & 1.3227 & 0.0000 & 1.2976 \\
\hline 70 & .6158 & 0.0607 & 0.1780 & 0.1044 & 0.0527 & 1.2976 & 0.0000 \\
\hline
\end{tabular}




\begin{tabular}{|c|c|c|c|c|c|c|}
\hline & $\mathrm{X} / \AA ̊$ & $\mathrm{Y} / \AA ̊$ & Z/Å & $\mathrm{q} / \mathrm{e}$ & WBI \\
\hline & $\mathrm{Si}$ & 0.000000 & 0.000000 & 0.416040 & +0.94 & 3.60 \\
\hline & B & 0.000000 & 1.941894 & 1.044308 & -0.33 & 3.67 \\
\hline & B & 0.000000 & 1.867475 & -0.475082 & -0.23 & 3.76 \\
\hline & B & 0.000000 & 0.763287 & -1.585526 & -0.23 & 3.44 \\
\hline & B & 0.000000 & -0.763287 & -1.585526 & -0.33 & 3.44 \\
\hline & B & 0.000000 & -1.867475 & -0.475082 & -0.04 & 3.76 \\
\hline & B & 0.000000 & -1.941894 & 1.044308 & -0.04 & 3.67 \\
\hline & $\mathrm{H}$ & 0.000000 & -2.303126 & 2.169224 & -0.36 & 1.00 \\
\hline & $\mathrm{H}$ & 0.000000 & 2.303126 & 2.169224 & -0.36 & 1.00 \\
\hline \multirow[t]{4}{*}{ Vibrational frequencies $/ \mathrm{cm}^{-1}$} & 181 & 183 & 278 & 370 & \multicolumn{2}{|c|}{392} \\
\hline & 409 & 527 & 584 & 600 & & \\
\hline & 690 & 734 & 943 & \multirow[t]{2}{*}{1210} & & \\
\hline & 1421 & 2697 & 2699 & & & \\
\hline
\end{tabular}

Wiberg bond Index matrix

$\begin{array}{llcrrrrrrr}\text { Atom } & 1 & 2 & 3 & 4 & 5 & 6 & 7 & 8 & 9\end{array}$

$\begin{array}{llllllllll}\text { 1. Si } & 0.0000 & 0.8169 & 0.5563 & 0.4172 & 0.4172 & 0.5563 & 0.8169 & 0.0080 & 0.0080\end{array}$

$\begin{array}{lllllllllll}\text { 2. B } & 0.8169 & 0.0000 & 1.6258 & 0.0462 & 0.0916 & 0.1032 & 0.0167 & 0.0020 & 0.9722\end{array}$

$\begin{array}{lllllllllll}\text { 3. } & \mathrm{B} & 0.5563 & 1.6258 & 0.0000 & 1.3285 & 0.0443 & 0.0938 & 0.1032 & 0.0009 & 0.0095\end{array}$

$\begin{array}{lllllllllll}\text { 4. B } & 0.4172 & 0.0462 & 1.3285 & 0.0000 & 1.5034 & 0.0443 & 0.0916 & 0.0062 & 0.0057\end{array}$

$\begin{array}{llllllllllll}\text { 5. B } & 0.4172 & 0.0916 & 0.0443 & 1.5034 & 0.0000 & 1.3285 & 0.0462 & 0.0057 & 0.0062\end{array}$

$\begin{array}{lllllllllll}\text { 6. } & \mathrm{B} & 0.5563 & 0.1032 & 0.0938 & 0.0443 & 1.3285 & 0.0000 & 1.6258 & 0.0095 & 0.0009\end{array}$

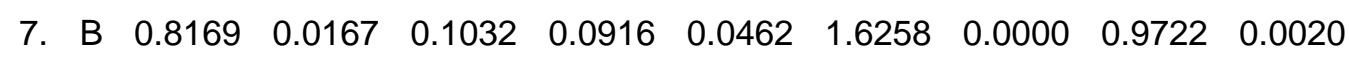

8. $\begin{array}{llllllllll}\mathrm{H} & 0.0080 & 0.0020 & 0.0009 & 0.0062 & 0.0057 & 0.0095 & 0.9722 & 0.0000 & 0.0002\end{array}$

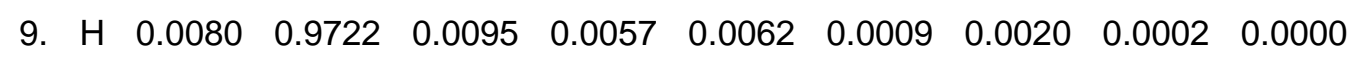

\section{1. $\mathrm{C}_{2 \mathrm{v}} \mathrm{B}_{4} \mathrm{C}_{2} \mathrm{H}_{2} \mathrm{Si}$}
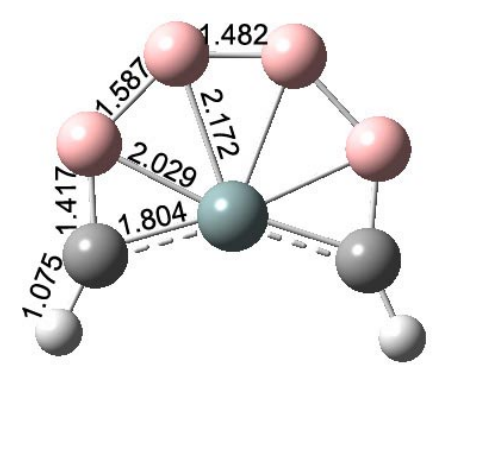

Vibrational frequencies $/ \mathrm{cm}^{-1} \quad 201$
423
X/Å

Si 0.000000

C $\quad 0.000000$

B $\quad 0.000000$

B $\quad 0.000000$

B $\quad 0.000000$

B $\quad 0.000000$

C $\quad 0.000000$

$\mathrm{H} \quad 0.000000$

H 0.000000

$$
\text { Y/Å }
$$

0.000000

1.724336

1.826119

0.740774

$-0.740774$

$-1.826119$

$-1.724336$

2.171124

$-2.171124$

$Z / \AA$
0.414288
0.943810
-0.469832
-1.626988
-1.626988
-0.469832
0.943810
1.921223
1.921223

204

$503 \quad 507$

$242 \quad 346$

$\begin{array}{cc}\text { q/e } & \text { WBI } \\ +1.52 & 3.46 \\ -1.08 & 3.70 \\ +0.37 & 3.36 \\ -0.32 & 3.76 \\ -0.32 & 3.76 \\ +0.37 & 3.36 \\ -1.08 & 3.70 \\ +0.27 & 0.93 \\ +0.27 & 0.93\end{array}$

$358 \quad 408$ 


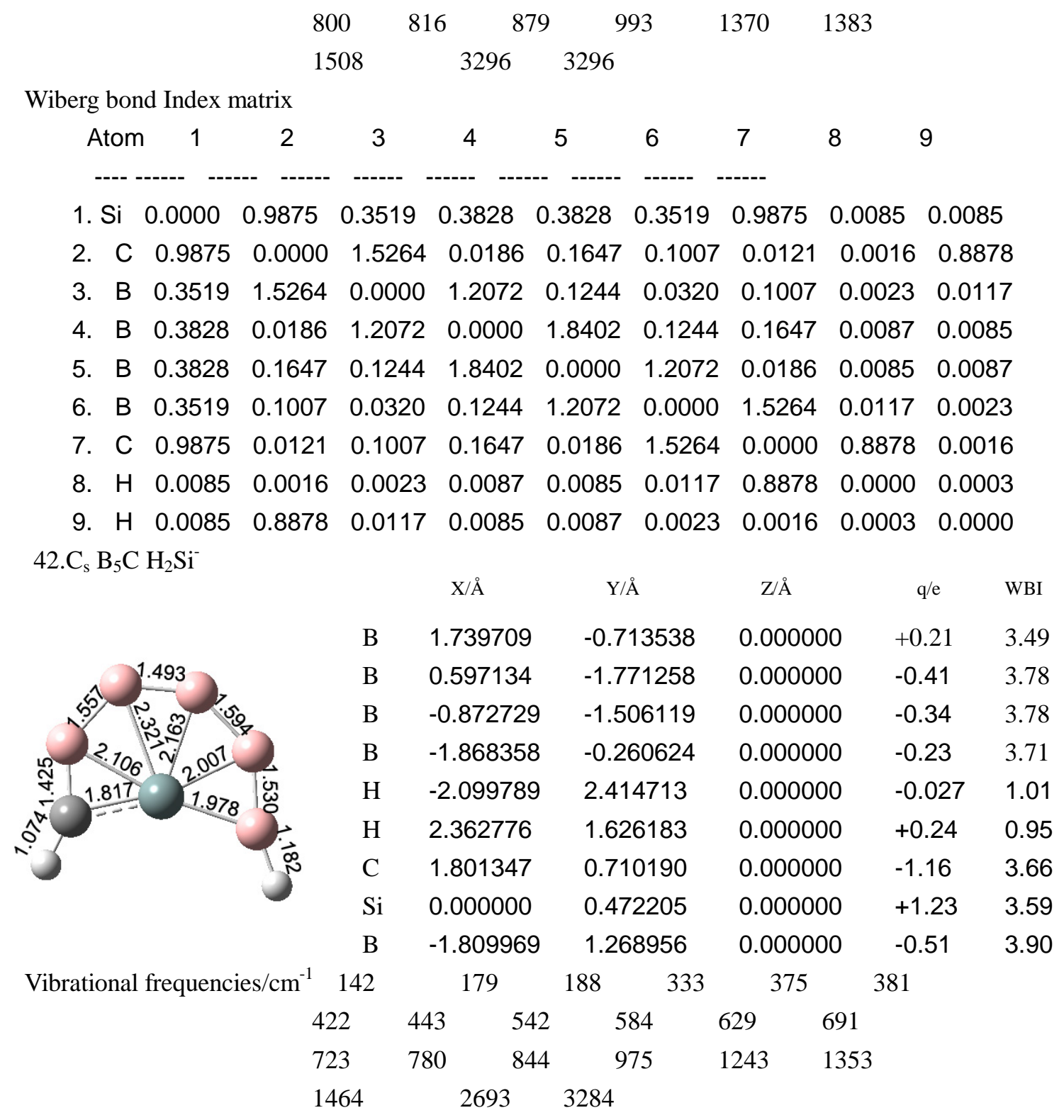

Wiberg bond Index matrix

\begin{tabular}{|c|c|c|c|c|c|c|c|c|c|}
\hline Atom & 1 & 2 & 3 & 4 & 5 & 6 & 7 & $y$ & \\
\hline & & & 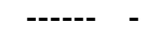 & 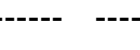 & -- & ------ & -- & & \\
\hline . $\mathrm{B}$ & 0.0000 & 1.3578 & 0.1662 & 0.0461 & 0.0020 & 0.0107 & 1.4859 & 0.2796 & 0.1372 \\
\hline$B$ & 1.3 & 0.0000 & 1.8227 & 0.0 & 0.0066 & 0.0091 & 0.0250 & 0.3150 & 0.1746 \\
\hline B & 0.1662 & 1.8227 & 0.0000 & 1.2350 & 0.0065 & 0.0106 & 0.1552 & 0.3658 & 0.0185 \\
\hline B & 0.0461 & 0.0657 & 1.2350 & 0.0000 & 0.0120 & 0.0020 & 0.0921 & 0.6493 & 1.6081 \\
\hline i. $\mathrm{H}$ & 0.0020 & 0.0066 & 0.0065 & 0.0 & 0.0000 & 0.0003 & 0.0013 & 0.0084 & 0.9682 \\
\hline . $\mathrm{H}$ & 0.0107 & 0.0091 & 0.0106 & 0.0020 & 0.0003 & 0.0 & 009 & 05 & 025 \\
\hline C & 1.4859 & 0.0250 & 0.1552 & 0.0 & 0.0 & 0.9009 & 0.0000 & 826 & 0.0132 \\
\hline & - & 0.3150 & 0.3658 & 0.6493 & 0.0084 & 0.0105 & 0.9826 & 0.0000 & 0.9817 \\
\hline B & 1 & 0.1746 & 0.0185 & 1.6081 & 0.9682 & .002 & 0. & 0.9817 & 000 \\
\hline
\end{tabular}


43. $\mathrm{C}_{2 \mathrm{v}} \quad \mathrm{B}_{5} \mathrm{Si}_{2} \mathrm{C}$

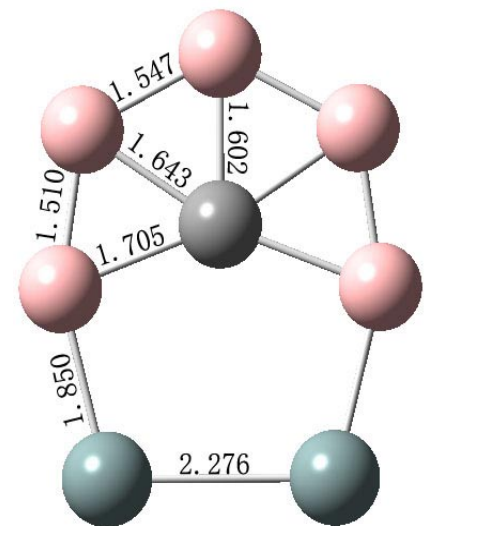

\begin{tabular}{|c|c|c|c|c|c|}
\hline & $\mathrm{X} / \AA ̊$ & Y/Å & Z/Å & $q / e$ & WBI \\
\hline $\mathrm{C}$ & 0.000000 & 0.000000 & 0.947253 & -0.64 & 3.90 \\
\hline $\mathrm{Si}$ & 0.000000 & 1.138176 & -1.441697 & +0.51 & 3.01 \\
\hline B & 0.000000 & 1.596763 & 0.350175 & -0.40 & 3.58 \\
\hline B & 0.000000 & 1.376787 & 1.843604 & +0.11 & 3.44 \\
\hline B & 0.000000 & 0.000000 & 2.549243 & +0.21 & 3.33 \\
\hline B & 0.000000 & -1.376787 & 1.843604 & +0.11 & 3.44 \\
\hline B & 0.000000 & -1.596763 & 0.350175 & -0.40 & 3.58 \\
\hline $\mathrm{Si}$ & 0.000000 & -1.138176 & -1.441697 & +0.51 & 3.01 \\
\hline
\end{tabular}

Vibrational frequencies $/ \mathrm{cm}^{-1}-2272$

$\begin{array}{ll}407 & 446 \\ 711 & 737\end{array}$

$\begin{array}{lllll}-288 & 198 & 208 & 309 & 384\end{array}$

711

737

464
1026

1026

$\begin{array}{cl}539 \quad 545 & 637 \\ 1213 & 1372\end{array}$

1411

Wiberg bond Index matrix
Atom 1
2
3
45
6
7

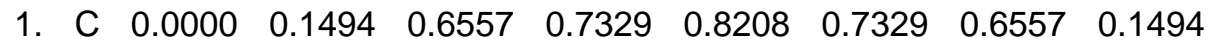
$\begin{array}{lllllllll}\text { 2. Si } & 0.1494 & 0.0000 & 1.2952 & 0.0379 & 0.1352 & 0.1362 & 0.0526 & 1.2018\end{array}$

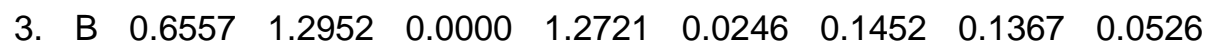
$\begin{array}{lllllllllll}\text { 4. } & \text { B } & 0.7329 & 0.0379 & 1.2721 & 0.0000 & 1.0943 & 0.0214 & 0.1452 & 0.1362\end{array}$
$\begin{array}{llllllllll}\text { 5. } & \text { B } & 0.8208 & 0.1352 & 0.0246 & 1.0943 & 0.0000 & 1.0943 & 0.0246 & 0.1352\end{array}$
6. $\begin{array}{llllllllll}\text { B } & 0.7329 & 0.1362 & 0.1452 & 0.0214 & 1.0943 & 0.0000 & 1.2721 & 0.0379\end{array}$

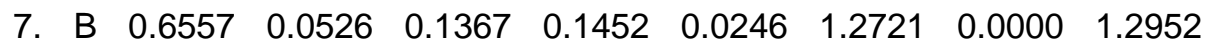

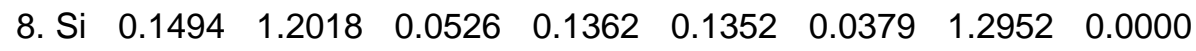

44. $\mathrm{C}_{2 \mathrm{v}} \quad \mathrm{B}_{5} \mathrm{Si}_{2} \mathrm{C}$

\begin{tabular}{|c|c|c|c|c|c|c|}
\hline & & $\mathrm{X} / \AA$ & Y/Å & Z/Å & $\mathrm{q} / \mathrm{e}$ & WBI \\
\hline & $\mathrm{C}$ & 0.000000 & 0.000000 & 1.044795 & -0.63 & 3.90 \\
\hline & $\mathrm{Si}$ & 0.000000 & 1.237529 & -1.493972 & +0.25 & 3.17 \\
\hline & B & 0.000000 & 1.535872 & 0.365079 & -0.51 & 3.63 \\
\hline & B & 0.000000 & 1.371985 & 1.882507 & +0.013 & 3.52 \\
\hline & B & 0.000000 & 0.000000 & 2.617319 & +0.11 & 3.40 \\
\hline & B & 0.000000 & -1.371985 & 1.882507 & +0.013 & 3.52 \\
\hline 2.475 & B & 0.000000 & -1.535872 & 0.365079 & -0.51 & 3.63 \\
\hline & $\mathrm{Si}$ & 0.000000 & -1.237529 & -1.493972 & +0.25 & 3.17 \\
\hline Vibrational frequencies $/ \mathrm{cm}^{-1}$ & -256 & 96 & 197 & 355 & 357 & \\
\hline 396 & & 485 & 535 & 575 & & \\
\hline 764 & & $105 \varepsilon$ & 119 & 132 & & 1337 \\
\hline
\end{tabular}


Wiberg bond Index matrix
$\begin{array}{lllrrrrrr}\text { Atom } 1 & 2 & 3 & 4 & 5 & 6 & 7 & 8\end{array}$

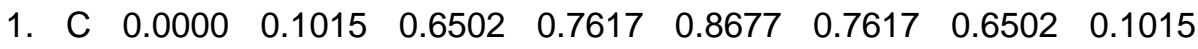

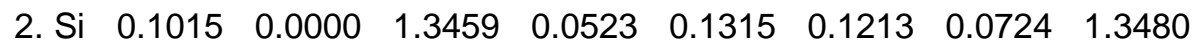
3. $\begin{array}{lllllllll}\mathrm{B} & 0.6502 & 1.3459 & 0.0000 & 1.2753 & 0.0240 & 0.1625 & 0.1075 & 0.0724\end{array}$

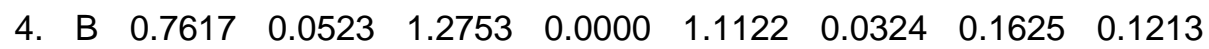

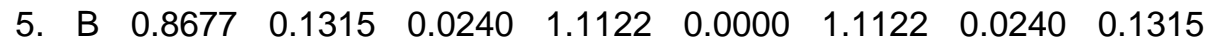

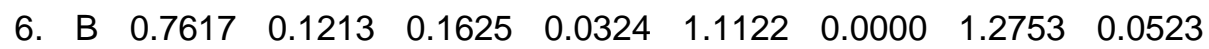

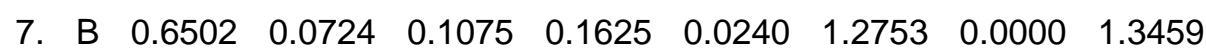

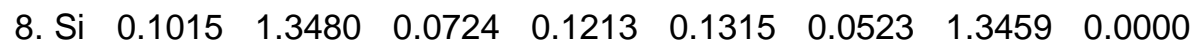

45. $\mathrm{C}_{2 \mathrm{v}} \quad \mathrm{B}_{5} \mathrm{Si}_{2} \mathrm{Si}$

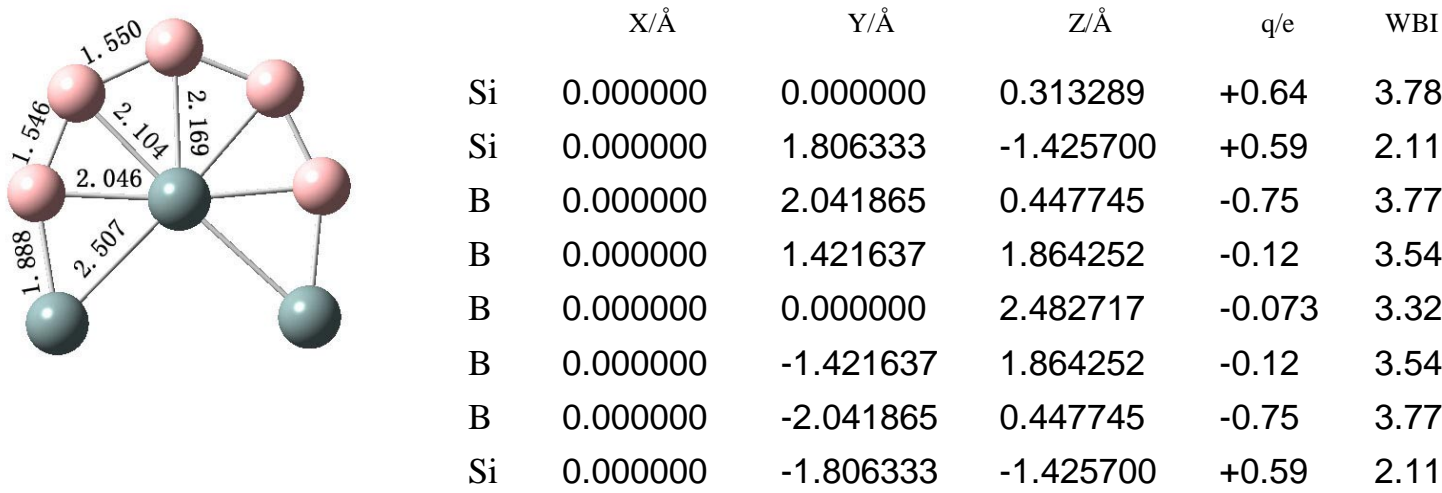

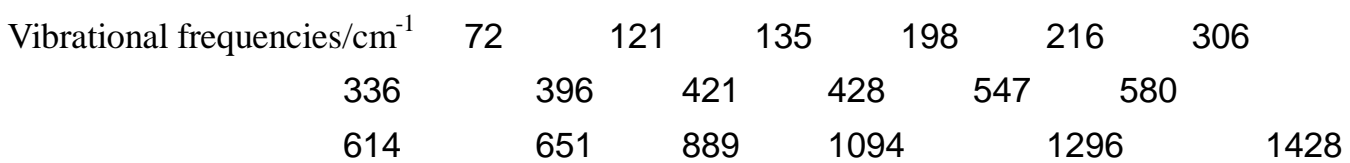

Wiberg bond Index matrix

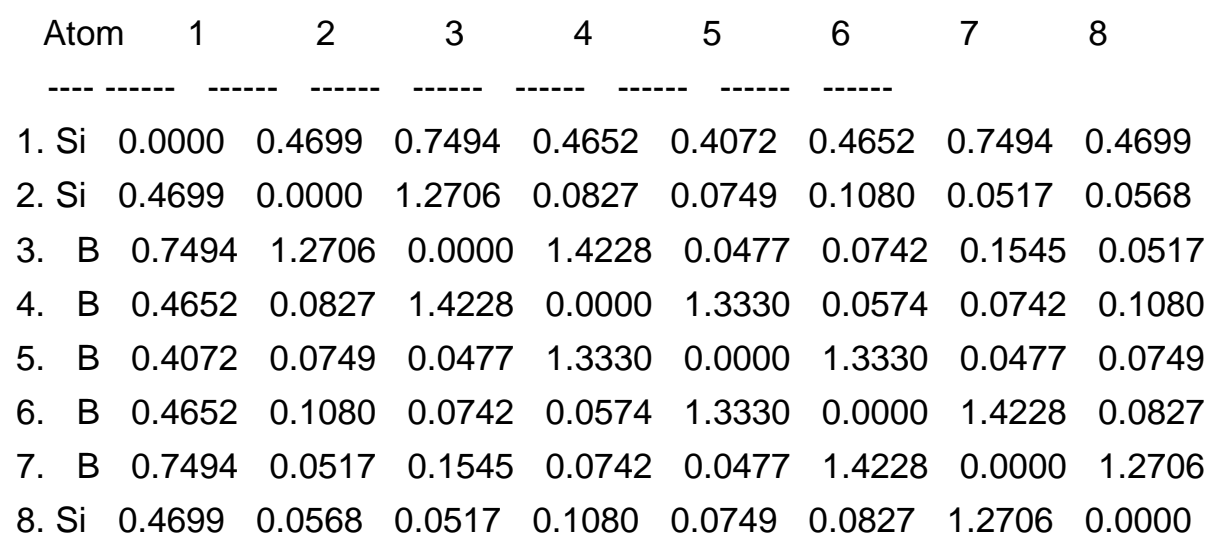


46. $\mathrm{C}_{2 \mathrm{v}} \mathrm{B}_{5} \mathrm{Si}_{2} \mathrm{Si}^{-}$

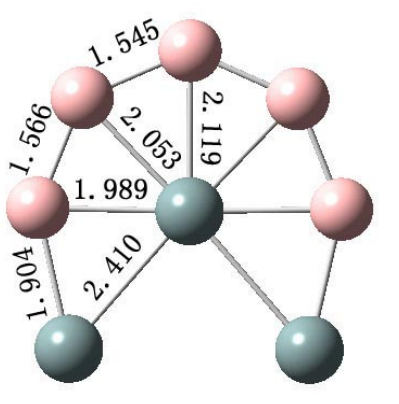

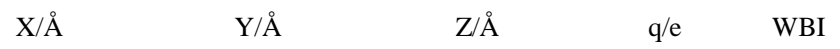

\begin{tabular}{|c|c|c|c|c|c|c|}
\hline $\mathrm{Si}$ & 0.000000 & 0.000000 & \multicolumn{2}{|c|}{0.376452} & +0.75 & 3.89 \\
\hline $\mathrm{Si}$ & 0.000000 & 1.574465 & \multicolumn{2}{|c|}{-1.447482} & +0.25 & 2.70 \\
\hline B & 0.000000 & 1.988502 & \multicolumn{2}{|c|}{0.411040} & -0.73 & 3.85 \\
\hline B & 0.000000 & 1.411115 & \multicolumn{2}{|c|}{1.867110} & -0.25 & 3.75 \\
\hline B & 0.000000 & 0.000000 & \multicolumn{2}{|c|}{2.495534} & -0.29 & 3.76 \\
\hline B & 0.000000 & -1.411115 & \multicolumn{2}{|c|}{1.867110} & -0.25 & 3.75 \\
\hline B & 0.000000 & -1.988502 & \multicolumn{2}{|c|}{0.411040} & -0.73 & 3.85 \\
\hline $\mathrm{Si}$ & 0.000000 & -1.574465 & \multicolumn{2}{|c|}{-1.447482} & +0.25 & 2.70 \\
\hline 101 & 140 & 146 & 233 & 270 & 386 & \\
\hline 393 & 427 & 447 & 453 & 588 & 624 & \\
\hline 724 & 891 & 1096 & \multicolumn{2}{|c|}{1215} & 1372 & \\
\hline
\end{tabular}

Wiberg bond Index matrix
Atom
1. Si 0.0000
23
4
$\begin{array}{lll}6 & 7 & 8\end{array}$
$\begin{array}{llllllllll}\text { 2. Si } & 0.5786 & 0.0000 & 1.3430 & 0.0465 & 0.1841 & 0.1964 & 0.0663 & 0.2807\end{array}$

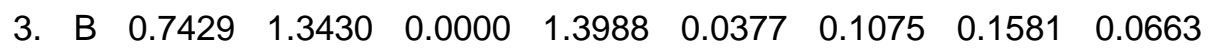
$\begin{array}{llllllllll}\text { 4. } & \text { B } & 0.4376 & 0.0465 & 1.3988 & 0.0000 & 1.4738 & 0.0854 & 0.1075 & 0.1964\end{array}$

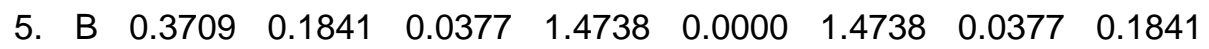

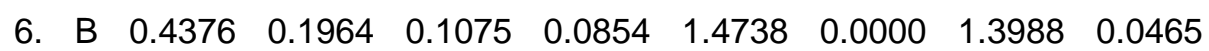
7. $\begin{array}{lllllllll}\text { B } & 0.7429 & 0.0663 & 0.1581 & 0.1075 & 0.0377 & 1.3988 & 0.0000 & 1.3430\end{array}$
8. Si $\begin{array}{lllllllll}0.5786 & 0.2807 & 0.0663 & 0.1964 & 0.1841 & 0.0465 & 1.3430 & 0.0000\end{array}$

47. $\mathrm{C}_{2 \mathrm{v}} \mathrm{B}_{5} \mathrm{Si}_{2} \mathrm{Ge}$

$\begin{array}{llccccc} & & \mathrm{X} / \AA & \mathrm{Y} / \AA & \mathrm{Z} / \AA & \mathrm{q} / \mathrm{e} & \text { WBI } \\ \mathrm{Ge} & 0.000000 & 0.000000 & 0.101173 & +0.56 & 3.58 \\ \mathrm{Si} & 0.000000 & 2.097436 & -1.394028 & +0.57 & 2.15 \\ \mathrm{~B} & 0.000000 & 2.146519 & 0.488662 & -0.72 & 3.73 \\ \mathrm{~B} & 0.000000 & 1.426009 & 1.862350 & -0.086 & 3.49 \\ \mathrm{~B} & 0.000000 & 0.000000 & 2.457024 & -0.082 & 3.28 \\ \mathrm{~B} & 0.000000 & -1.426009 & 1.862350 & -0.086 & 3.49 \\ \mathrm{~B} & 0.000000 & -2.146519 & 0.488662 & -0.72 & 3.73 \\ \mathrm{Si} & 0.000000 & -2.097436 & -1.394028 & +0.57 & 2.15\end{array}$

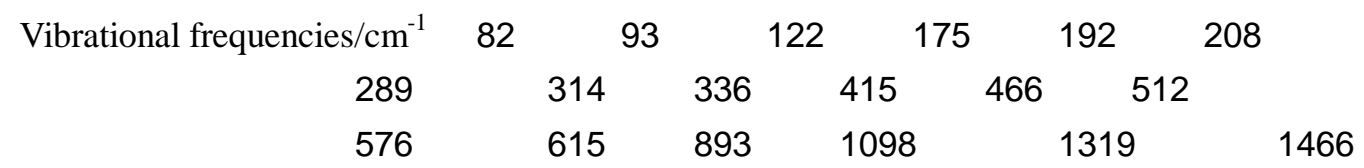


Wiberg bond Index matrix

\begin{tabular}{lccccccccc}
\multicolumn{1}{l}{ Atom } & 1 & 2 & 3 & 4 & 5 & 6 & 7 & 8 \\
& Ge & 0.0000 & 0.4837 & 0.7141 & 0.4139 & 0.3523 & 0.4139 & 0.7141 & 0.4837 \\
2. Si & 0.4837 & 0.0000 & 1.3350 & 0.0878 & 0.0680 & 0.1163 & 0.0460 & 0.0096 \\
3. B & 0.7141 & 1.3350 & 0.0000 & 1.3944 & 0.0506 & 0.0483 & 0.1442 & 0.0460 \\
4. & B & 0.4139 & 0.0878 & 1.3944 & 0.0000 & 1.3467 & 0.0810 & 0.0483 & 0.1163 \\
5. & B & 0.3523 & 0.0680 & 0.0506 & 1.3467 & 0.0000 & 1.3467 & 0.0506 & 0.0680 \\
6. & B & 0.4139 & 0.1163 & 0.0483 & 0.0810 & 1.3467 & 0.0000 & 1.3944 & 0.0878 \\
7. B & 0.7141 & 0.0460 & 0.1442 & 0.0483 & 0.0506 & 1.3944 & 0.0000 & 1.3350 \\
8. Si & 0.4837 & 0.0096 & 0.0460 & 0.1163 & 0.0680 & 0.0878 & 1.3350 & 0.0000
\end{tabular}

48. $\mathrm{C}_{2 \mathrm{v}} \mathrm{B}_{5} \mathrm{Si}_{2} \mathrm{Ge}^{-}$

\begin{tabular}{|c|c|c|c|c|c|}
\hline & $\mathrm{X} / \AA ̊$ & $\mathrm{Y} / \AA ̊$ & Z/Å & $q / e$ & WBI \\
\hline $\mathrm{Ge}$ & 0.000000 & 0.000000 & 0.064170 & +0.61 & 3.72 \\
\hline $\mathrm{Si}$ & 0.000000 & 2.033138 & -1.379089 & +0.26 & 2.62 \\
\hline B & 0.000000 & 2.103618 & 0.496507 & -0.73 & 3.82 \\
\hline B & 0.000000 & 1.389809 & 1.896224 & -0.18 & 3.67 \\
\hline B & 0.000000 & 0.000000 & 2.526750 & -0.29 & 3.72 \\
\hline B & 0.000000 & -1.389809 & 1.896224 & -0.18 & 3.67 \\
\hline B & 0.000000 & -2.103618 & 0.496507 & -0.73 & 3.82 \\
\hline $\mathrm{Si}$ & 0.000000 & -2.033138 & -1.379089 & +0.26 & 2.62 \\
\hline
\end{tabular}

\begin{tabular}{ccccccccc} 
Vibrational frequencies $/ \mathrm{cm}^{-1}$ & 99 & \multicolumn{1}{c}{100} & 118 & 206 & 208 & 223 \\
308 & 350 & 377 & 400 & 489 & 514 & \\
573 & 625 & 920 & 1115 & 1268 & 1476
\end{tabular}

Wiberg bond Index matrix

\begin{tabular}{|c|c|c|c|c|c|c|c|c|}
\hline Atom & 1 & 2 & 3 & 4 & 5 & 6 & 7 & 8 \\
\hline--- & 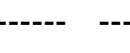 & ----- & --.. & --- & - & ----- & & \\
\hline $\mathrm{Ge}$ & 0.0000 & 0.6483 & 0.7474 & 0.3348 & 0.2581 & 0.3348 & 0.7474 & 0.6483 \\
\hline $\mathrm{Si}$ & 0.6483 & 0.0000 & 1.4812 & 0.0452 & 0.1581 & 0.2066 & 0.0437 & 0.0407 \\
\hline 3. $B$ & 0.7474 & 1.4812 & 0.0000 & 1.3240 & 0.0432 & 0.0483 & 0.1351 & 0.0437 \\
\hline 4. $B$ & 0.3348 & 0.0452 & 1.3240 & 0.0000 & 1.5305 & 0.1793 & 0.0483 & 0.2066 \\
\hline 5. B & 0.2581 & 0.1581 & 0.0432 & 1.5305 & 0.0000 & 1.5305 & 0.0432 & 0.1581 \\
\hline 6. B & 0.3348 & 0.2066 & 0.0483 & 0.1793 & 1.5305 & 0.0000 & 1.3240 & 0.0452 \\
\hline 7. B & 0.7474 & 0.0437 & 0.1351 & 0.0483 & 0.0432 & 1.3240 & 0.0000 & 1.4812 \\
\hline 3. $\mathrm{Si}$ & .6483 & 0.0407 & 0.0437 & 0.2066 & 0.1581 & 0.0452 & 1.4812 & 0.0000 \\
\hline
\end{tabular}




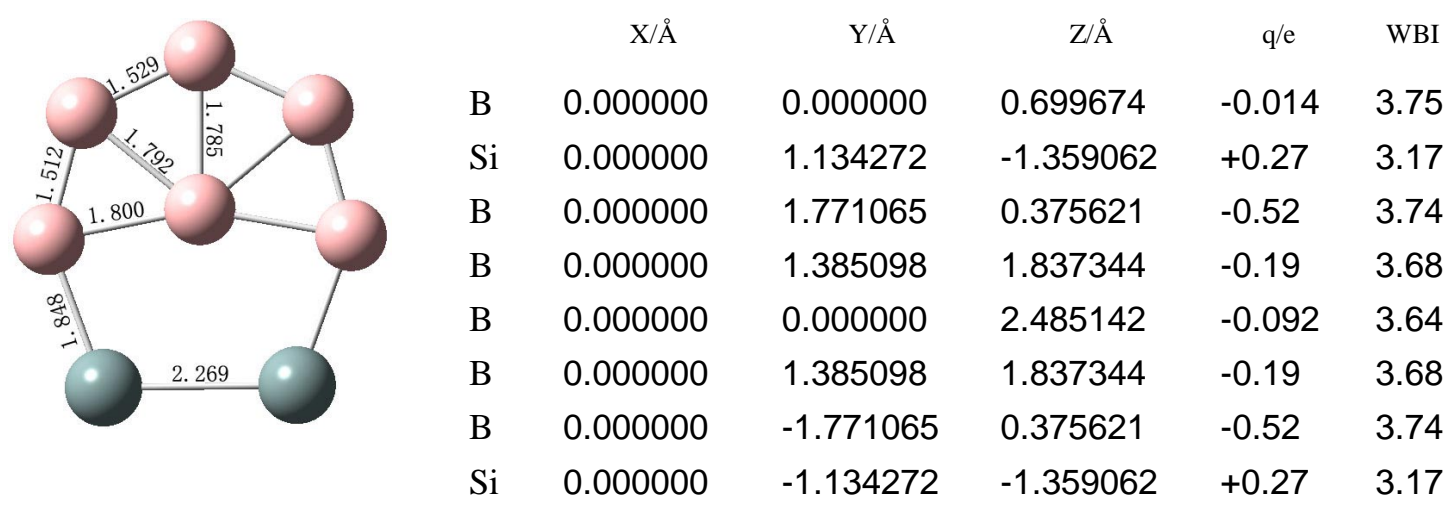

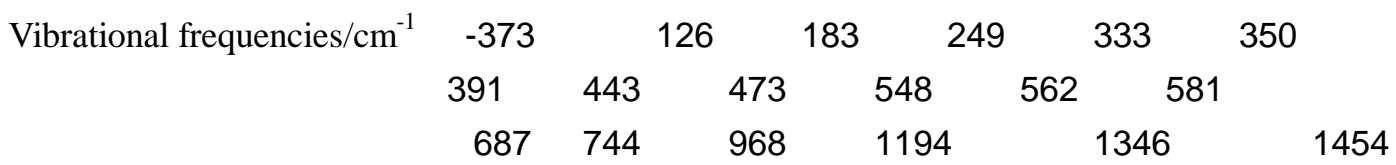

Wiberg bond Index matrix

$\begin{array}{lllrrrrrr}\text { Atom } 1 & 2 & 3 & 4 & 5 & 6 & 7 & 8 \\ - & & & \end{array}$

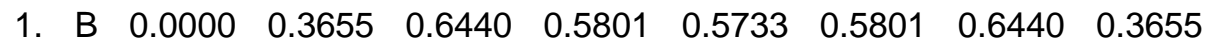

$\begin{array}{lllllllll}\text { 2. Si } & 0.3655 & 0.0000 & 1.3052 & 0.0283 & 0.1607 & 0.1758 & 0.0624 & 1.0670\end{array}$

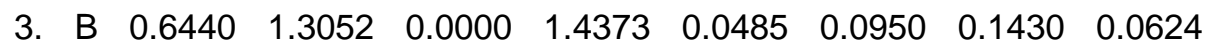

$\begin{array}{llllllllll}4 . \mathrm{B} & 0.5801 & 0.0283 & 1.4373 & 0.0000 & 1.3264 & 0.0377 & 0.0950 & 0.1758\end{array}$

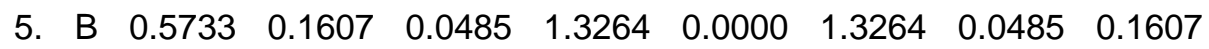

6. $\begin{array}{llllllllll}\text { B } & 0.5801 & 0.1758 & 0.0950 & 0.0377 & 1.3264 & 0.0000 & 1.4373 & 0.0283\end{array}$

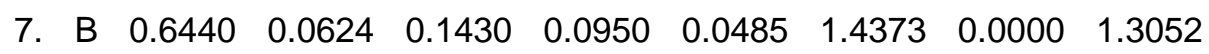

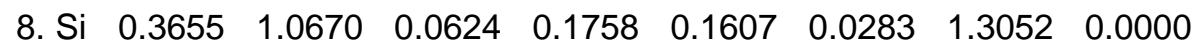

$50 . \mathrm{C}_{2 \mathrm{v}} \mathrm{B}_{5} \mathrm{Si}_{2} \mathrm{Al}^{-}$

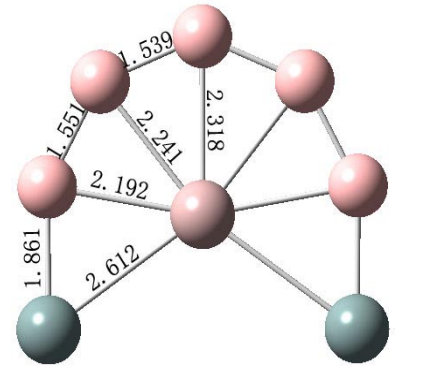

\begin{tabular}{lcclrr} 
& $\mathrm{X} / \AA$ & $\mathrm{Y} / \AA$ & \multicolumn{1}{c}{$\mathrm{Z} / \AA$} & $\mathrm{q} / \mathrm{e}$ & WBI \\
$\mathrm{Al}$ & 0.000000 & 0.000000 & 0.139357 & +1.02 & 2.80 \\
$\mathrm{Si}$ & 0.000000 & 2.143488 & -1.353216 & +0.24 & 2.44 \\
$\mathrm{~B}$ & 0.000000 & 2.160925 & 0.507785 & -0.88 & 3.86 \\
$\mathrm{~B}$ & 0.000000 & 1.422433 & 1.871196 & -0.24 & 3.64 \\
$\mathrm{~B}$ & 0.000000 & 0.000000 & 2.457716 & -0.26 & 3.49 \\
$\mathrm{~B}$ & 0.000000 & -1.422433 & 1.871196 & -0.24 & 3.64 \\
$\mathrm{~B}$ & 0.000000 & -2.160925 & 0.507785 & -0.88 & 3.86 \\
$\mathrm{Si}$ & 0.000000 & -2.143488 & -1.353216 & +0.24 & 2.44
\end{tabular}

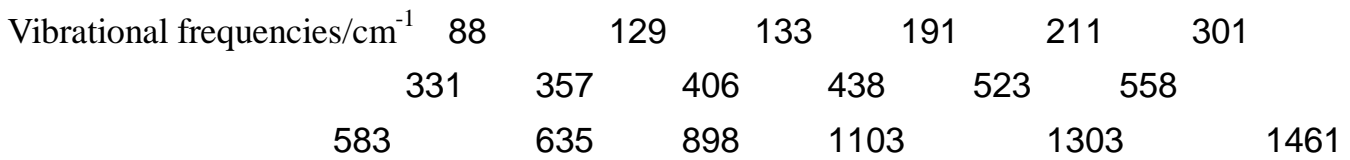


Wiberg bond Index matrix

\begin{tabular}{|c|c|c|c|c|c|c|c|c|}
\hline Atom & $n$ & 2 & 3 & 4 & 5 & 6 & & \\
\hline & . & & 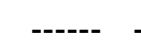 & 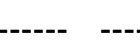 & 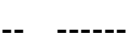 & ------ & & \\
\hline & Oח & 7 & 7 & 0.27 & 0.2 & 2 & & \\
\hline II & 0.4101 & & & & & & & \\
\hline B & 0.5267 & & 000 & & & & & 30 \\
\hline B & .2712 & & 0 & & & & & \\
\hline D & .24 & 35 & 9 & & 0. & 2 & & 73 \\
\hline & 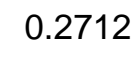 & & & & & & & 1102 \\
\hline D & 0.52 & 0 & 6 & & & & & 94 \\
\hline & 4787 & 0420 & 0300 & 1149 & 0.0735 & 0.1102 & 1.5941 & 0.0000 \\
\hline
\end{tabular}

51. $\mathrm{C}_{2 \mathrm{v}} \mathrm{B}_{5} \mathrm{Si}_{2} \mathrm{Ga}^{-}$

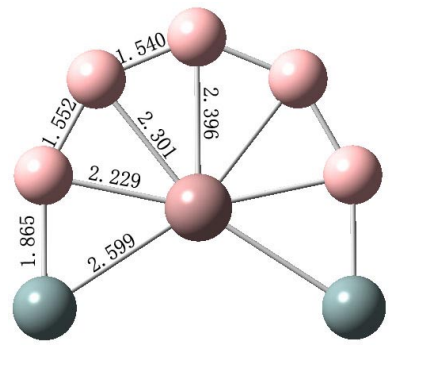

\begin{tabular}{|c|c|c|c|c|c|}
\hline & X/Å & Y/Å & Z/Å & $\mathrm{q} / \mathrm{e}$ & WBI \\
\hline $\mathrm{Ga}$ & 0.000000 & 0.000000 & 0.058331 & +0.76 & 2.96 \\
\hline $\mathrm{Si}$ & 0.000000 & 2.182187 & -1.352513 & +0.25 & 2.42 \\
\hline B & 0.000000 & 2.182081 & 0.512884 & -0.82 & 3.82 \\
\hline B & 0.000000 & 1.423494 & 1.866281 & -0.20 & 3.60 \\
\hline B & 0.000000 & 0.000000 & 2.454088 & -0.22 & 3.44 \\
\hline B & 0.000000 & -1.423494 & 1.866281 & -0.20 & 3.60 \\
\hline B & 0.000000 & -2.182081 & 0.512884 & -0.82 & 3.82 \\
\hline $\mathrm{Si}$ & 0.000000 & -2.182187 & -1.352513 & +0.25 & 2.4 \\
\hline
\end{tabular}

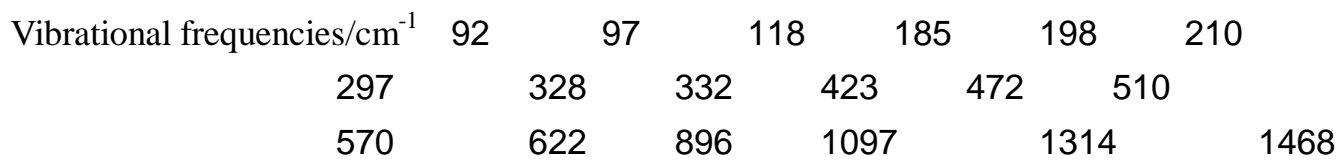

Wiberg bond Index matrix

\begin{tabular}{|c|c|c|c|c|c|c|c|c|}
\hline Atom & $n$ & 2 & $\checkmark$ & 4 & $\checkmark$ & 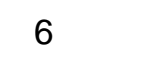 & & \\
\hline & & & & - & & & & \\
\hline C & 0.0 & 5012 & 5 & 0.2 & 0.2577 & $0.2 s$ & & \\
\hline Si & .5012 & 0 & 627 & 0.10 & & 0.1 & & \\
\hline B & 0.5 & & & 1.4 & & & & \\
\hline$B$ & 0.2 & & & & & & & \\
\hline B & 25 & 6 & & 1.4 & & & & \\
\hline$B$ & 0.2925 & 37 & 5 & 0. & 35 & 0.00 & & 0 \\
\hline & 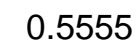 & 4 & 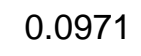 & 5 & 0. & & & 5627 \\
\hline 0 & 5012 & 267 & 314 & 1187 & 0.0716 & 0.1096 & 1.5627 & 0.0000 \\
\hline
\end{tabular}




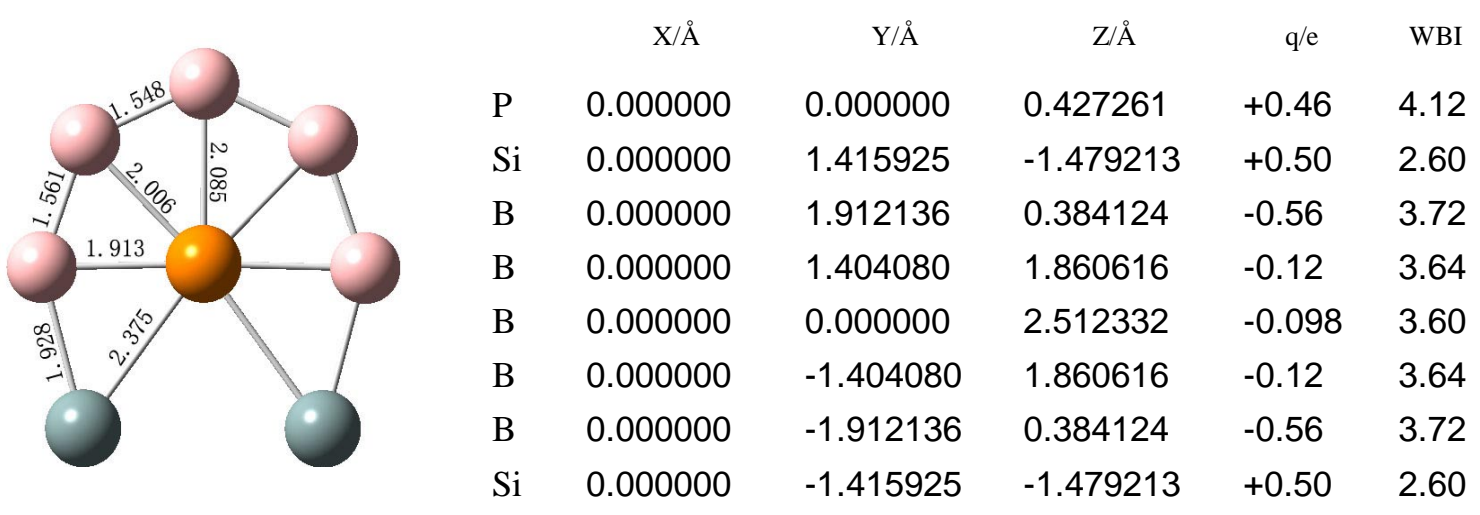

$\begin{array}{rrrrrrrrrrr}\text { Vibrational frequencies/cm } & 120 & & 141 & & 159 & 218 & 221 & & 346 & \\ 362 & & 396 & & 408 & 443 & 551 & 596 & \\ & 613 & & 717 & 893 & 1110 & 1234 & 1379\end{array}$

Wiberg bond Index matrix

$\begin{array}{lllrrrrrr}\text { Atom } & 1 & 2 & 3 & 4 & 5 & 6 & 7 & 8 \\ - & & & \end{array}$

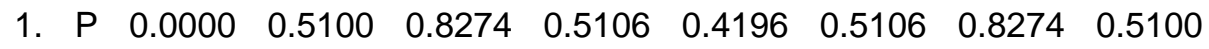

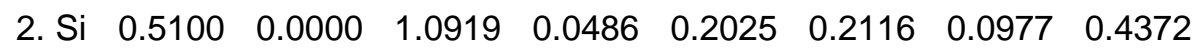

3. $\begin{array}{llllllllll} & 0.8274 & 1.0919 & 0.0000 & 1.3065 & 0.0258 & 0.1505 & 0.2203 & 0.0977\end{array}$

$\begin{array}{llllllllll}\text { 4. } & \mathrm{B} & 0.5106 & 0.0486 & 1.3065 & 0.0000 & 1.3640 & 0.0488 & 0.1505 & 0.2116\end{array}$

5. B \begin{tabular}{llllllllll}
\hline & 0.4196 & 0.2025 & 0.0258 & 1.3640 & 0.0000 & 1.3640 & 0.0258 & 0.2025
\end{tabular}

6. B $\begin{array}{llllllllll}0.5106 & 0.2116 & 0.1505 & 0.0488 & 1.3640 & 0.0000 & 1.3065 & 0.0486\end{array}$

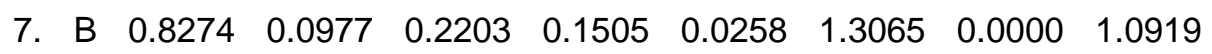

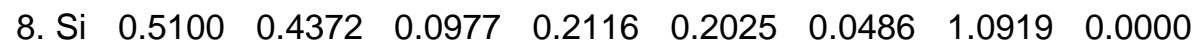

$53 . \mathrm{C}_{2 \mathrm{v}} \mathrm{B}_{5} \mathrm{Si}_{2} \mathrm{As}$

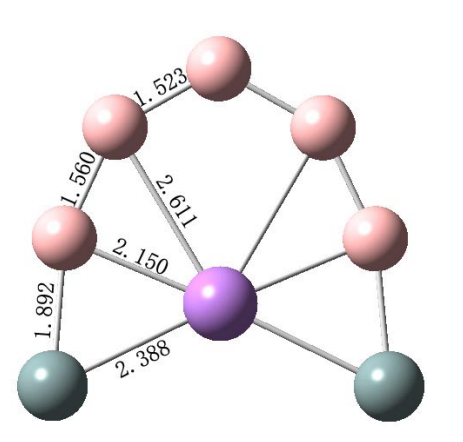

\begin{tabular}{lcclll} 
& $\mathrm{X} / \AA$ & $\mathrm{Y} / \AA$ & \multicolumn{1}{c}{ Z/A } & \multicolumn{1}{c}{$\mathrm{q} / \mathrm{e}$} & WBI \\
$\mathrm{As}$ & 0.000000 & 0.000000 & 0.190465 & +0.20 & 3.71 \\
$\mathrm{Si}$ & 0.000000 & 2.143573 & 1.242250 & +0.58 & 2.43 \\
$\mathrm{~B}$ & 0.000000 & 1.981430 & -0.643089 & -0.65 & 3.73 \\
$\mathrm{~B}$ & 0.000000 & 1.325475 & -2.058908 & -0.0046 & 3.50 \\
$\mathrm{~B}$ & 0.000000 & 0.000000 & -2.809675 & -0.054 & 3.47 \\
$\mathrm{~B}$ & 0.000000 & -1.325475 & -2.058908 & -0.0046 & 3.50 \\
$\mathrm{~B}$ & 0.000000 & -1.981430 & -0.643089 & -0.65 & 3.73 \\
$\mathrm{Si}$ & 0.000000 & -2.143573 & 1.242250 & +0.58 & 2.43
\end{tabular}

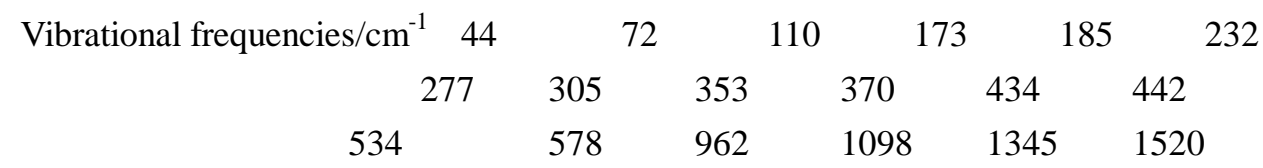


Wiberg bond Index matrix

$\begin{array}{lcccccccccc}\text { Atom } & 1 & 2 & 3 & 4 & 5 & 6 & 7 & 8 \\ ----- & ----- & ----- & ----- & ----- & ----- & ---- & ---- & ---- & & \\ \text { 1. } & \text { As } & 0.0000 & 0.7227 & 0.7993 & 0.2454 & 0.1763 & 0.2454 & 0.7993 & 0.7227 \\ \text { 2. } & \text { Si } & 0.7227 & 0.0000 & 1.2565 & 0.0566 & 0.0952 & 0.1485 & 0.1297 & 0.0224 \\ \text { 3. } & \text { B } & 0.7993 & 1.2565 & 0.0000 & 1.3087 & 0.0787 & 0.0236 & 0.1318 & 0.1297 \\ \text { 4. } & \text { B } & 0.2454 & 0.0566 & 1.3087 & 0.0000 & 1.4714 & 0.2409 & 0.0236 & 0.1485 \\ \text { 5. } & \text { B } & 0.1763 & 0.0952 & 0.0787 & 1.4714 & 0.0000 & 1.4714 & 0.0787 & 0.0952 \\ \text { 6. } & \text { B } & 0.2454 & 0.1485 & 0.0236 & 0.2409 & 1.4714 & 0.0000 & 1.3087 & 0.0566 \\ \text { 7. } & \text { B } & 0.7993 & 0.1297 & 0.1318 & 0.0236 & 0.0787 & 1.3087 & 0.0000 & 1.2565 \\ \text { 8. } & \text { Si } & 0.7227 & 0.0224 & 0.1297 & 0.1485 & 0.0952 & 0.0566 & 1.2565 & 0.0000\end{array}$

54. $\mathrm{D}_{8 \mathrm{~h}} \mathrm{~B}_{8} \mathrm{Si}$

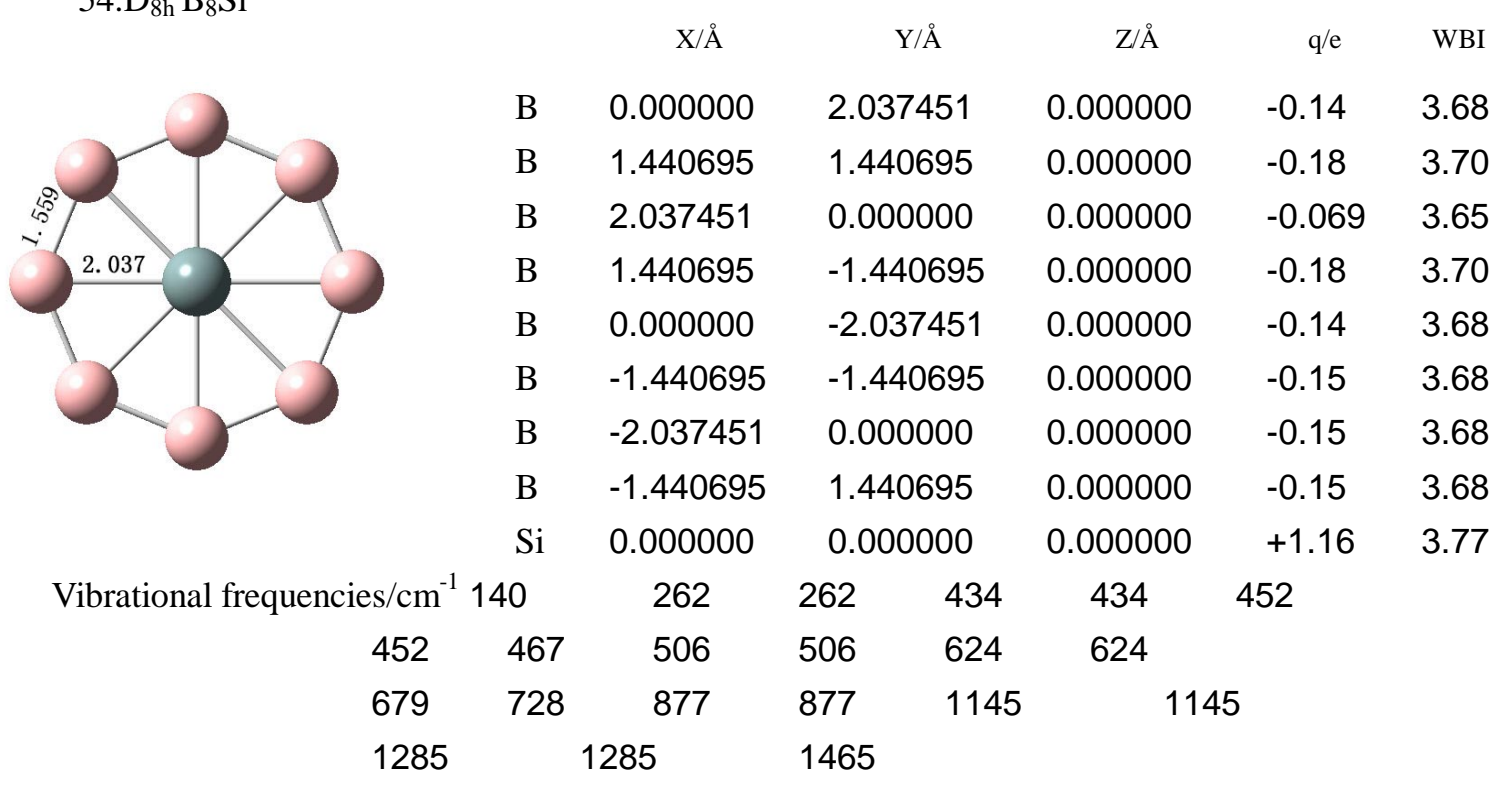

Wiberg bond Index matrix

\begin{tabular}{|c|c|c|c|c|c|c|c|c|c|}
\hline Atom & 1 & 2 & 3 & 4 & & & & \\
\hline & -- & . & 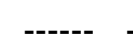 & 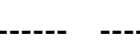 & 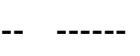 & ------ & & & \\
\hline 1. $B$ & 0.0000 & 4 & 0 & 0.0637 & 0.1 & 0631 & 0 & 7 & \\
\hline B & 1.3 & 00 & 2 & & & & & & \\
\hline 3. $B$ & 0.0 & & 0.0 & 1.3 & 0. & 0.0 & & & 41 \\
\hline 4. $B$ & 0.0 & & 2 & 0.0 & & & & & \\
\hline 5. B & 0.16 & 37 & 0.0 & 1.3 & 00 & 77 & 77 & 31 & 704 \\
\hline 6. $\mathrm{B}$ & 0.0 & & 0 & 0.0 & & 0 & & & 001 \\
\hline 7. B & 0. & & & & & & & 94 & 99 \\
\hline 8. $B$ & 1.3 & 0 . & 0 & & 31 & 4 & 94 & 000 & 701 \\
\hline $9 \mathrm{Si}$ & 4704 & 0.4766 & 0.4641 & 0.4766 & 0.4704 & 0.4701 & 0.4699 & 0.4701 & 0.0000 \\
\hline
\end{tabular}




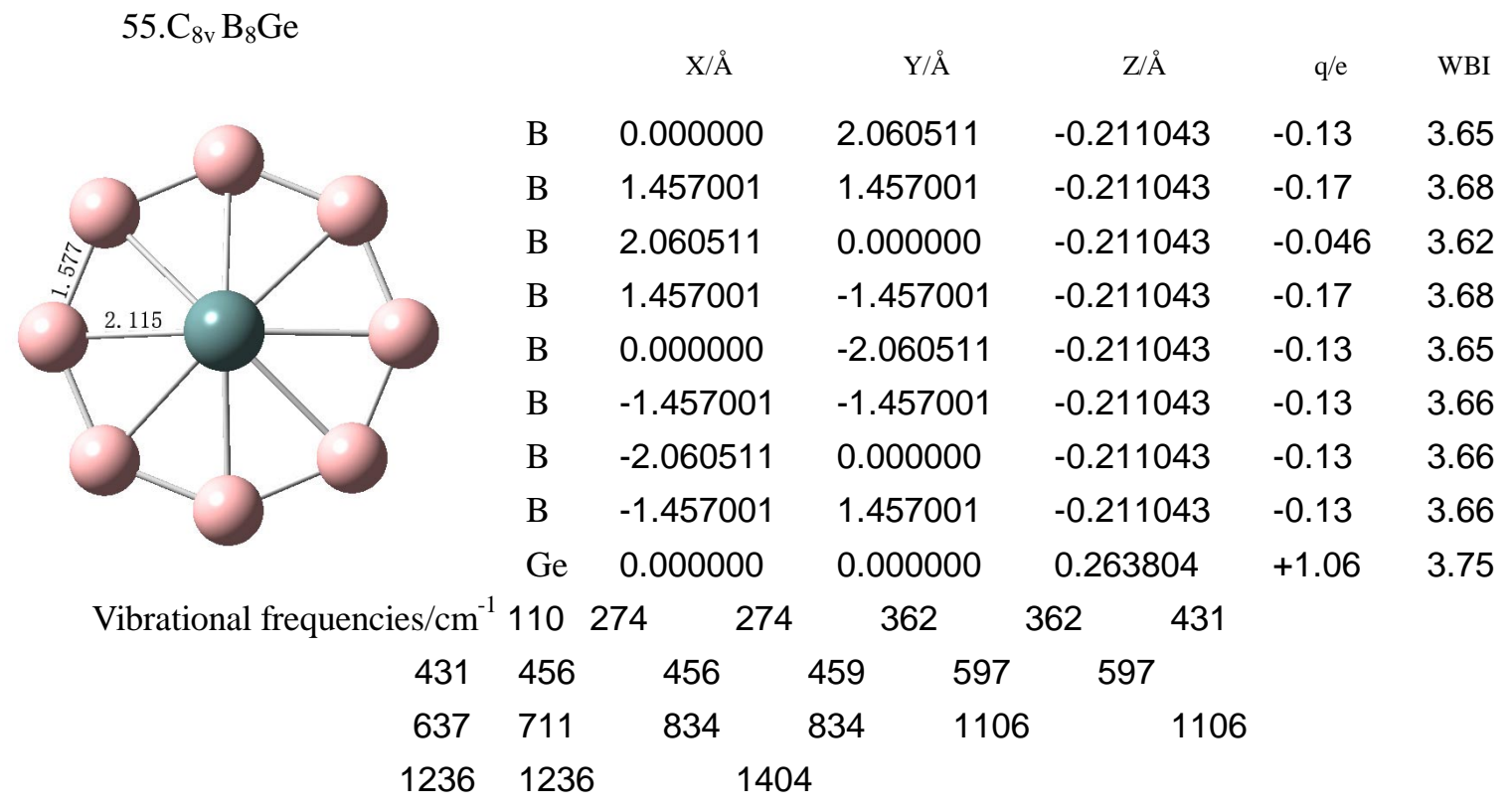

Wiberg bond Index matrix

$\begin{array}{lllrrrrrrr}\text { Atom } & 1 & 2 & 3 & 4 & 5 & 6 & 7 & 8 & 9\end{array}$

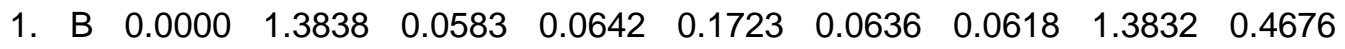

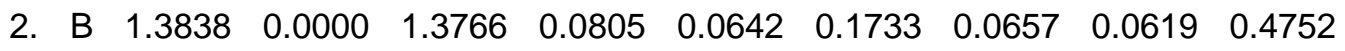

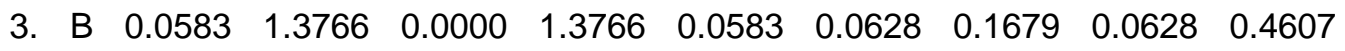

4. $\begin{array}{lllllllllll}\mathrm{B} & 0.0642 & 0.0805 & 1.3766 & 0.0000 & 1.3838 & 0.0619 & 0.0657 & 0.1733 & 0.4752\end{array}$

$\begin{array}{llllllllllll}\text { 5. B } & 0.1723 & 0.0642 & 0.0583 & 1.3838 & 0.0000 & 1.3832 & 0.0618 & 0.0636 & 0.4676\end{array}$

6. B $\begin{array}{lllllllllll}0.0636 & 0.1733 & 0.0628 & 0.0619 & 1.3832 & 0.0000 & 1.3828 & 0.0615 & 0.4676\end{array}$

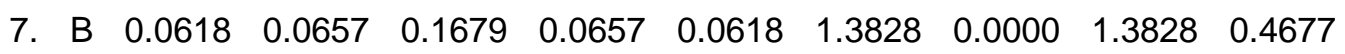

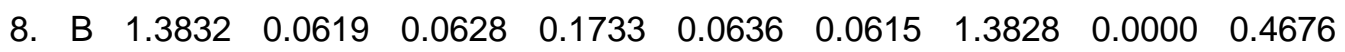

$\begin{array}{llllllllll}\text { 9. } \mathrm{Ge} & 0.4676 & 0.4752 & 0.4607 & 0.4752 & 0.4676 & 0.4676 & 0.4677 & 0.4676 & 0.0000\end{array}$ 56. $\mathrm{C}_{8 \mathrm{v}} \mathrm{B}_{8} \mathrm{Al}^{-}$

$\begin{array}{ccccccr} & \mathrm{X} / \AA & \mathrm{Y} / \AA & \mathrm{Z} / \AA & \mathrm{q} / \mathrm{e} & \mathrm{WBI} \\ \mathrm{B} & 0.000000 & 2.047007 & -0.128674 & -0.31 & 3.80 \\ \mathrm{~B} & 1.447453 & 1.447453 & -0.128674 & -0.31 & 3.80 \\ \mathrm{~B} & 2.047007 & 0.000000 & -0.128674 & -0.31 & 3.80 \\ \mathrm{~B} & 1.447453 & -1.447453 & -0.128674 & -0.35 & 3.82 \\ \mathrm{~B} & 0.000000 & -2.047007 & -0.128674 & -0.23 & 3.78 \\ \mathrm{~B} & -1.447453 & -1.447453 & -0.128674 & -0.35 & 3.82 \\ \mathrm{~B} & -2.047007 & 0.000000 & -0.128674 & -0.31 & 3.80 \\ \mathrm{~B} & -1.447453 & 1.447453 & -0.128674 & -0.31 & 3.80 \\ \mathrm{Al} & 0.000000 & 0.000000 & 0.395921 & +1.47 & 2.52\end{array}$

$\begin{array}{rrrrrrr}\text { Vibrational frequencies } / \mathrm{cm}^{-1} & 174 & 282 & 282 & 435 & 435 & 462 \\ 465 & 465 & 501 & 501 & 629 & 629 & \end{array}$




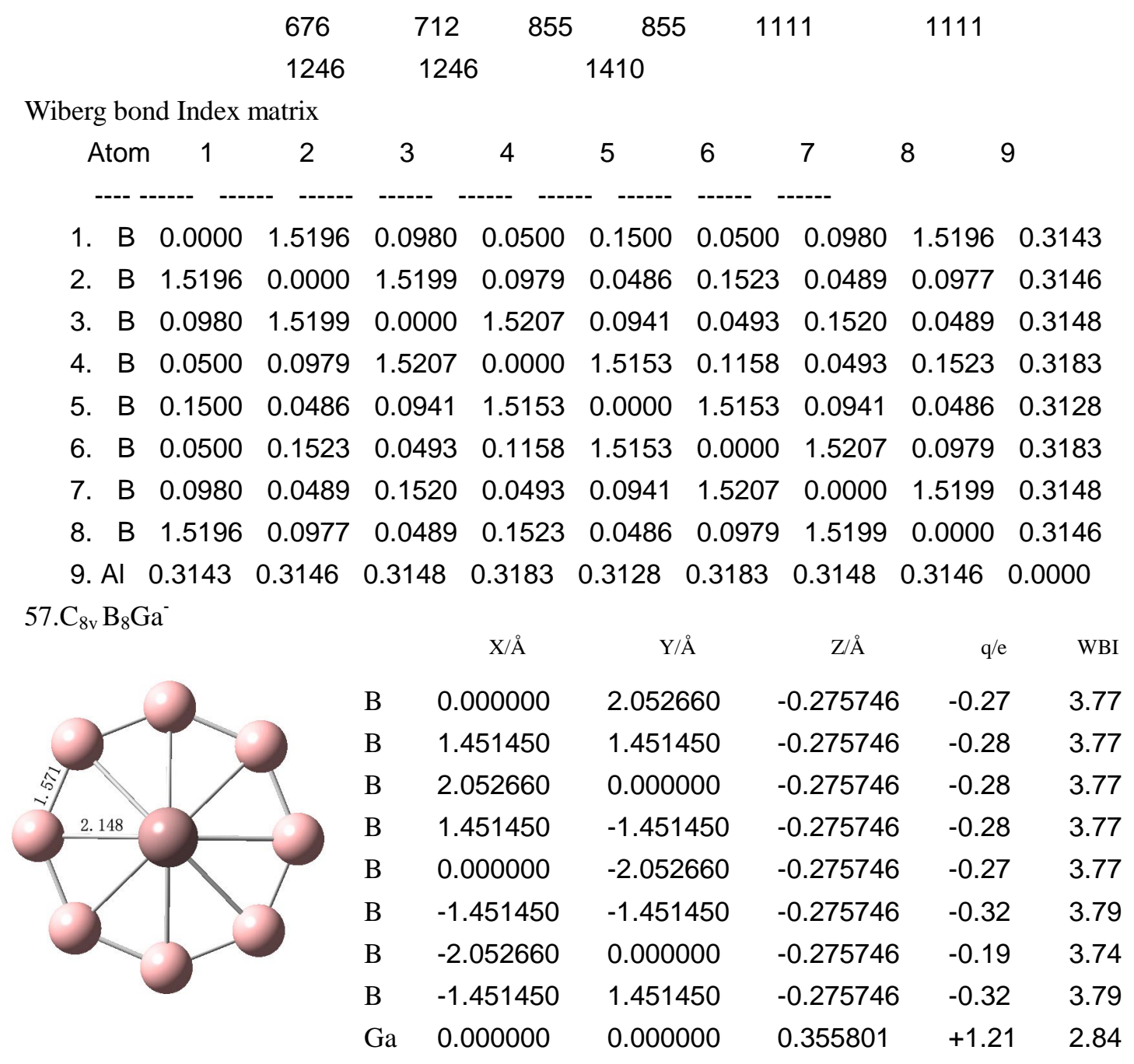

\begin{tabular}{rrrrrrrr} 
Vibrational frequencies/ $\mathrm{cm}^{-1}$ & 139 & 279 & 279 & 357 & 357 & 421 \\
421 & 446 & 450 & 450 & 584 & 584 \\
620 & 704 & 834 & 834 & 1106 & & 1107 \\
1244 & 1244 & \multicolumn{2}{c}{1400} & & &
\end{tabular}

Wiberg bond Index matrix
$\begin{array}{llllllllll}\text { Atom } & 1 & 2 & 3 & 4 & 5 & 6 & 7 & 8 & 9\end{array}$

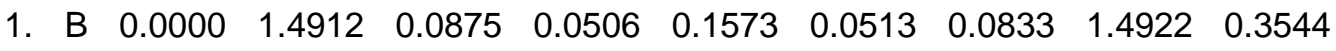

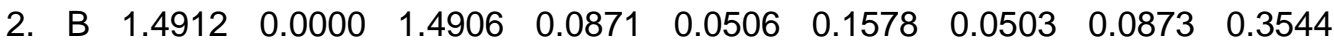

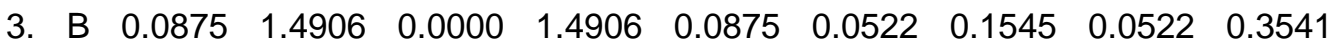
$\begin{array}{lllllllllll}\text { 4. B } & 0.0506 & 0.0871 & 1.4906 & 0.0000 & 1.4912 & 0.0873 & 0.0503 & 0.1578 & 0.3544\end{array}$
$\begin{array}{lllllllllll}\text { 5. B } & 0.1573 & 0.0506 & 0.0875 & 1.4912 & 0.0000 & 1.4922 & 0.0833 & 0.0513 & 0.3544\end{array}$
6. $\begin{array}{lllllllllll}\mathrm{B} & 0.0513 & 0.1578 & 0.0522 & 0.0873 & 1.4922 & 0.0000 & 1.4864 & 0.1078 & 0.3588\end{array}$

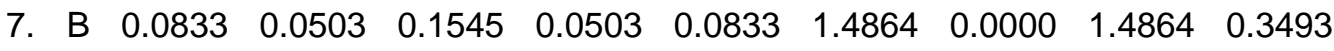
$\begin{array}{lllllllllll}\text { 8. B } & 1.4922 & 0.0873 & 0.0522 & 0.1578 & 0.0513 & 0.1078 & 1.4864 & 0.0000 & 0.3588\end{array}$ 


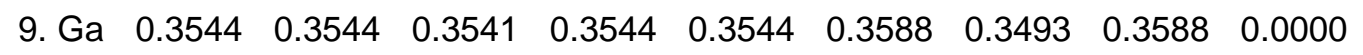
58. $\mathrm{D}_{8 \mathrm{~h}} \mathrm{~B}_{8} \mathrm{P}^{+}$

$\begin{array}{ccccccc} & \mathrm{X} / \AA & \mathrm{Y} / \AA & \mathrm{Z} / \AA & \mathrm{q} / \mathrm{e} & \mathrm{WBI} \\ \mathrm{B} & 0.000000 & 2.038572 & 0.000000 & +0.024 & 3.52 \\ \mathrm{~B} & 1.441488 & 1.441488 & 0.000000 & +0.023 & 3.52 \\ \mathrm{~B} & 2.038572 & 0.000000 & 0.000000 & +0.023 & 3.52 \\ \mathrm{~B} & 1.441488 & -1.441488 & 0.000000 & +0.023 & 3.52 \\ \mathrm{~B} & 0.000000 & -2.038572 & 0.000000 & +0.024 & 3.52 \\ \mathrm{~B} & -1.441488 & -1.441488 & 0.000000 & -0.013 & 3.54 \\ \mathrm{~B} & -2.038572 & 0.000000 & 0.000000 & +0.10 & 3.48 \\ \mathrm{~B} & -1.441488 & 1.441488 & 0.000000 & -0.013 & 3.54 \\ \mathrm{P} & 0.000000 & 0.000000 & 0.000000 & +0.81 & 4.17\end{array}$

$\begin{array}{ccccccccc}\text { Vibrational frequencies } / \mathrm{cm}^{-1} 184 & 231 & 231 & 380 & 380 & 386 \\ 386 & 394 & 394 & 424 & 521 & 521 \\ 574 & 706 & 850 & 850 & 1171 & 1171 \\ 1322 & 1322 & 1508 & & & & \end{array}$

Wiberg bond Index matrix

$\begin{array}{lllrrrrrrr}\text { Atom } & 1 & 2 & 3 & 4 & 5 & 6 & 7 & 8 & 9\end{array}$

1. $\begin{array}{llllllllll}\mathrm{B} & 0.0000 & 1.2753 & 0.0396 & 0.0841 & 0.1994 & 0.0851 & 0.0364 & 1.2763 & 0.5207\end{array}$

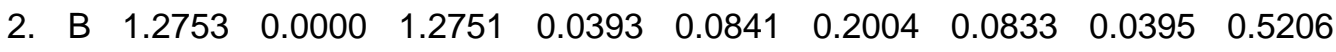

$\begin{array}{lllllllllll}3 . & B & 0.0396 & 1.2751 & 0.0000 & 1.2751 & 0.0396 & 0.0862 & 0.1954 & 0.0862 & 0.5204\end{array}$

$\begin{array}{lllllllllll}\text { 4. } & \mathrm{B} & 0.0841 & 0.0393 & 1.2751 & 0.0000 & 1.2753 & 0.0395 & 0.0833 & 0.2004 & 0.5206\end{array}$

$\begin{array}{lllllllllll}\text { 5. B } & 0.1994 & 0.0841 & 0.0396 & 1.2753 & 0.0000 & 1.2763 & 0.0364 & 0.0851 & 0.5207\end{array}$

6. B $\begin{array}{llllllllll}0.0851 & 0.2004 & 0.0862 & 0.0395 & 1.2763 & 0.0000 & 1.2681 & 0.0565 & 0.5302\end{array}$

7. $\begin{array}{lllllllllll}\text { B } & 0.0364 & 0.0833 & 0.1954 & 0.0833 & 0.0364 & 1.2681 & 0.0000 & 1.2681 & 0.5054\end{array}$

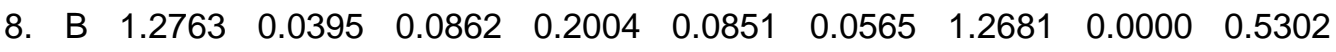

$\begin{array}{llllllllllll}\text { 9. } & \mathrm{P} & 0.5207 & 0.5206 & 0.5204 & 0.5206 & 0.5207 & 0.5302 & 0.5054 & 0.5302 & 0.0000\end{array}$ 59. $\mathrm{C}_{8 \mathrm{v}} \mathrm{B}_{8} \mathrm{As}^{+}$

$\begin{array}{ccccccr} & & \mathrm{X} / \AA & \mathrm{Y} / \AA & \mathrm{Z} / \AA & \mathrm{q} / \mathrm{e} & \text { WBI } \\ \mathrm{B} & 0.000000 & 2.076792 & -0.181555 & +0.10 & 3.48 \\ \mathrm{~B} & 1.468514 & 1.468514 & -0.181555 & -0.030 & 3.55 \\ \mathrm{~B} & 2.076792 & 0.000000 & -0.181555 & +0.012 & 3.52 \\ \mathrm{~B} & 1.468514 & -1.468514 & -0.181555 & +0.010 & 3.52 \\ \mathrm{~B} & 0.000000 & -2.076792 & -0.181555 & +0.011 & 3.52 \\ \mathrm{~B} & -1.468514 & -1.468514 & -0.181555 & +0.010 & 3.52 \\ \mathrm{~B} & -2.076792 & 0.000000 & -0.181555 & +0.012 & 3.52 \\ \mathrm{~B} & -1.468514 & 1.468514 & -0.181555 & -0.030 & 3.55 \\ \mathrm{As} & 0.000000 & 0.000000 & 0.220066 & +0.90 & 4.04\end{array}$




$\begin{array}{crrlllll}\text { Vibrational frequencies/cm } & 90 & 265 & 265 & 326 & 326 & 417 & \\ 417 & 432 & 434 & 434 & 558 & 558 & \\ 594 & 698 & 810 & 810 & 1098 & & 1098 \\ 1222 & 1222 & 1398 & & & & \end{array}$

Wiberg bond Index matrix

\begin{tabular}{|c|c|c|c|c|c|c|c|c|c|}
\hline tom & 1 & 2 & 3 & 4 & 5 & 6 & 7 & 0 & \\
\hline & & & & & & & & & \\
\hline & & & & & & & & & \\
\hline B & .27 & 000 & 7 & 0. & 351 & 2002 & 38 & 86 & \\
\hline 3. $B$ & 0.0 & 1 & 0 & 1 & 8 & 30 & 90 & 38 & 44 \\
\hline 1. B & 0.0 & 0 & 1.2867 & 0 & 64 & 5 & 30 & 0 & 52 \\
\hline 5. B & 0.15 & 1 & 8 & 4 & 0 & 34 & 98 & 0.0 & 057 \\
\hline 6. $B$ & 0.08 & 2002 & 0830 & 0 & 4 & .0000 & .2867 & 0.0 & 052 \\
\hline 7. $B$ & 0.0 & .0838 & 0 & & & & 0.0000 & 1.2877 & 044 \\
\hline 8. $B$ & 0 & 6 & 8 & 0. & 51 & 0.0400 & 877 & 0.0000 & 134 \\
\hline A & 0.4926 & 5134 & .5044 & 0.5052 & 57 & 0.5052 & 0.5044 & 0.5134 & 0.0000 \\
\hline
\end{tabular}
60. $\mathrm{C}_{2 \mathrm{v}} \mathrm{B}_{7} \mathrm{CSi}^{+}$

$\begin{array}{ccccccc} & & \mathrm{X} / \AA & \mathrm{Y} / \AA & \mathrm{Z} / \AA & \mathrm{q} / \mathrm{e} & \text { WBI } \\ \mathrm{B} & 0.000000 & 0.000000 & 2.055169 & +0.084 & 3.51 \\ \mathrm{~B} & 0.000000 & 1.464014 & 1.434477 & -0.075 & 3.63 \\ \mathrm{~B} & 0.000000 & 2.046894 & -0.007261 & -0.030 & 3.61 \\ \mathrm{~B} & 0.000000 & 1.373696 & -1.437747 & +0.35 & 3.40 \\ \mathrm{~B} & 0.000000 & -1.373696 & -1.437747 & +0.35 & 3.40 \\ \mathrm{~B} & 0.000000 & -2.046894 & -0.007261 & -0.030 & 3.61 \\ \mathrm{~B} & 0.000000 & -1.464014 & 1.434477 & -0.075 & 3.63 \\ \mathrm{Si} & 0.000000 & 0.000000 & 0.062676 & +1.23 & 3.71 \\ \mathrm{C} & 0.000000 & 0.000000 & -1.841334 & -0.80 & 3.76\end{array}$

\begin{tabular}{rrrrrrrrr} 
Vibrational frequencies $/ \mathrm{cm}^{-1}$ & 68 & \multicolumn{2}{c}{271} & 272 & 440 & 453 & 468 \\
469 & 495 & 505 & 556 & 638 & 662 \\
708 & 748 & 882 & 911 & 1105 & 1174 \\
1301 & \multicolumn{2}{c}{1346} & 1539 & & &
\end{tabular}

Wiberg bond Index matrix
Atom 2
3 45 5 $6 \quad 7$

7 89

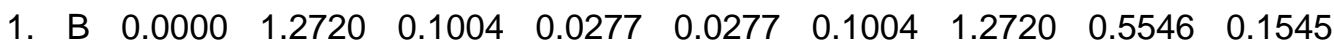

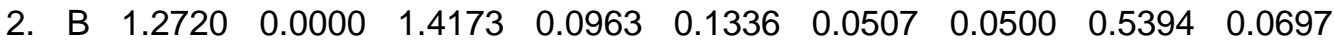
3. $\begin{array}{llllllllllll}\text { B } & 0.1004 & 1.4173 & 0.0000 & 1.2680 & 0.1072 & 0.1778 & 0.0507 & 0.4423 & 0.0424\end{array}$

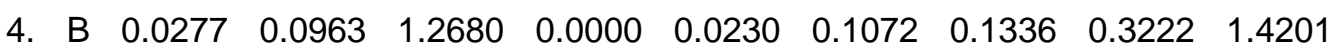




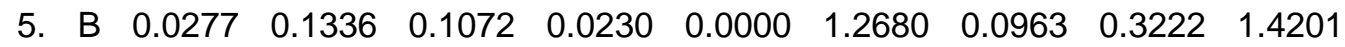

$\begin{array}{llllllllllll}\text { 6. } & \text { B } & 0.1004 & 0.0507 & 0.1778 & 0.1072 & 1.2680 & 0.0000 & 1.4173 & 0.4423 & 0.0424\end{array}$

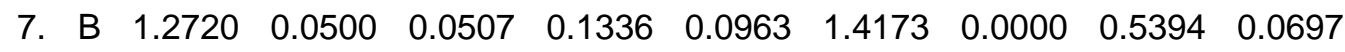

$\begin{array}{llllllllll}\text { 8. Si } & 0.5546 & 0.5394 & 0.4423 & 0.3222 & 0.3222 & 0.4423 & 0.5394 & 0.0000 & 0.5449\end{array}$

9. $\begin{array}{llllllllll}\mathrm{C} & 0.1545 & 0.0697 & 0.0424 & 1.4201 & 1.4201 & 0.0424 & 0.0697 & 0.5449 & 0.0000\end{array}$ 61. $\mathrm{D}_{2 \mathrm{~h}} \mathrm{~B}_{6} \mathrm{C}_{2} \mathrm{Si}^{2+}$

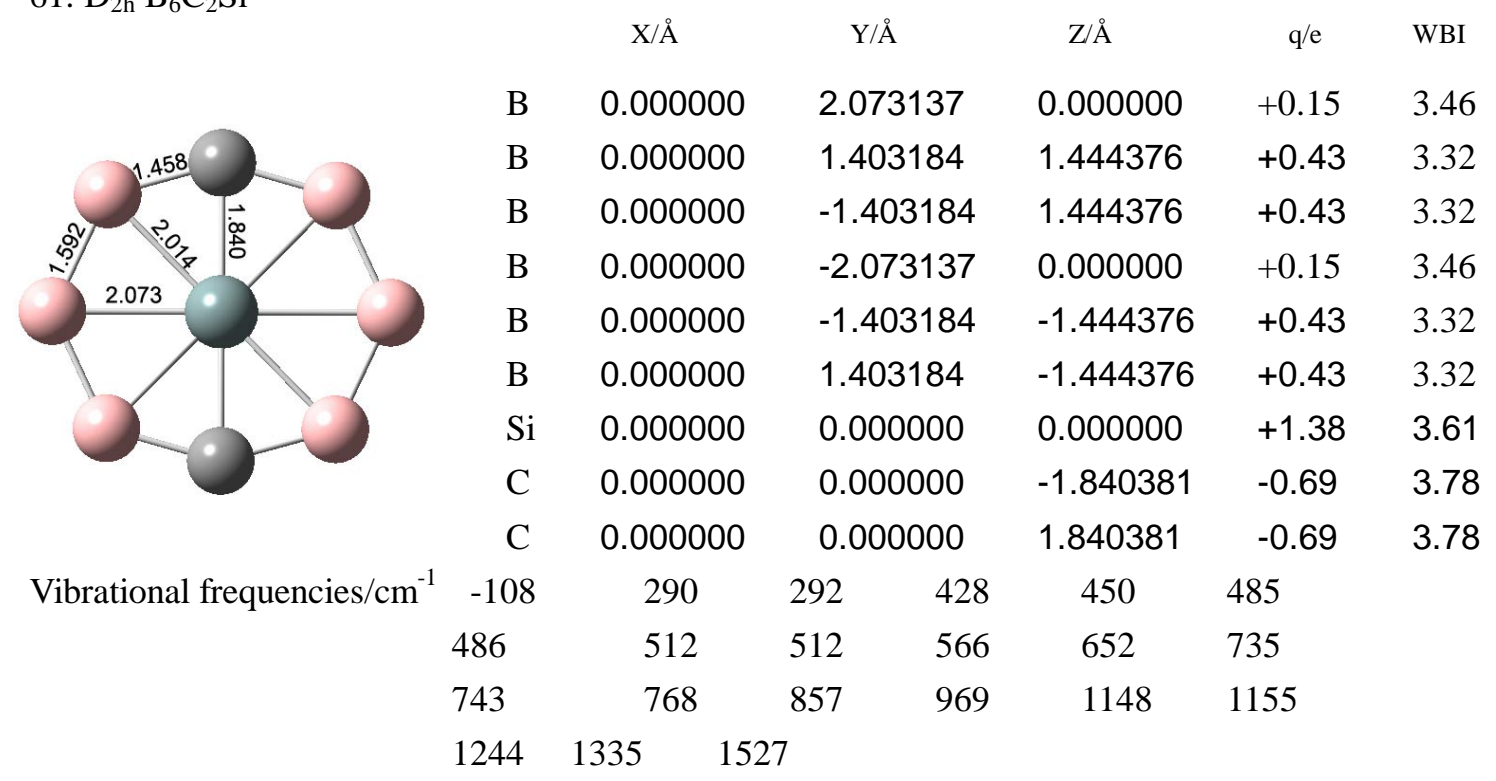

Wiberg bond Index matrix

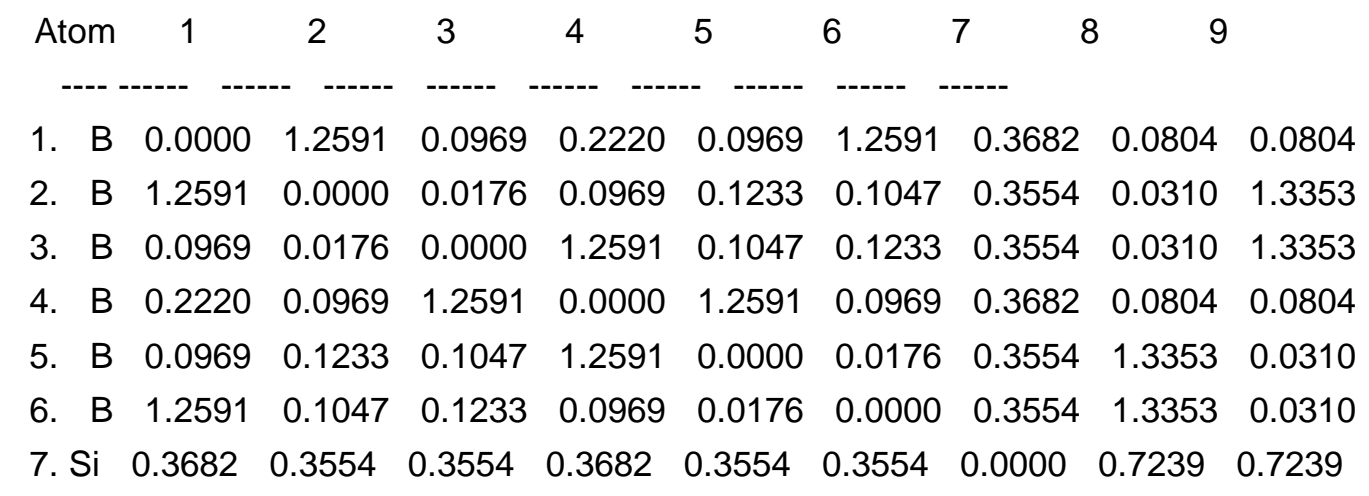




\section{Part II: Comparison of different isomers of $\mathrm{B}_{2} \mathrm{Si}_{2} \mathrm{Si}^{-}$and $\mathrm{B}_{3} \mathrm{Si}_{2} \mathrm{Si}^{-}$}

\section{1. $\mathrm{C}_{2 \mathrm{v}} \mathrm{B}_{2} \mathrm{Si}_{2} \mathrm{Si}^{-}$}

(1). $\mathrm{C}_{2 \mathrm{v}} \mathrm{B}_{2} \mathrm{Si}_{2} \mathrm{Si}^{-}$

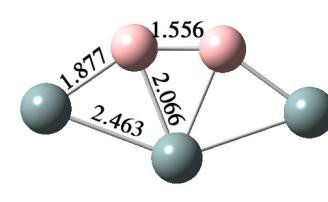

(2). $\mathrm{C}_{\mathrm{s}} \mathrm{B}_{2} \mathrm{Si}_{2} \mathrm{Si}^{-}$

Vibrational frequencies $/ \mathrm{cm}^{-1} 110$ 628

$\mathrm{HF}=-918.2183391$

Wiberg bond Index matrix

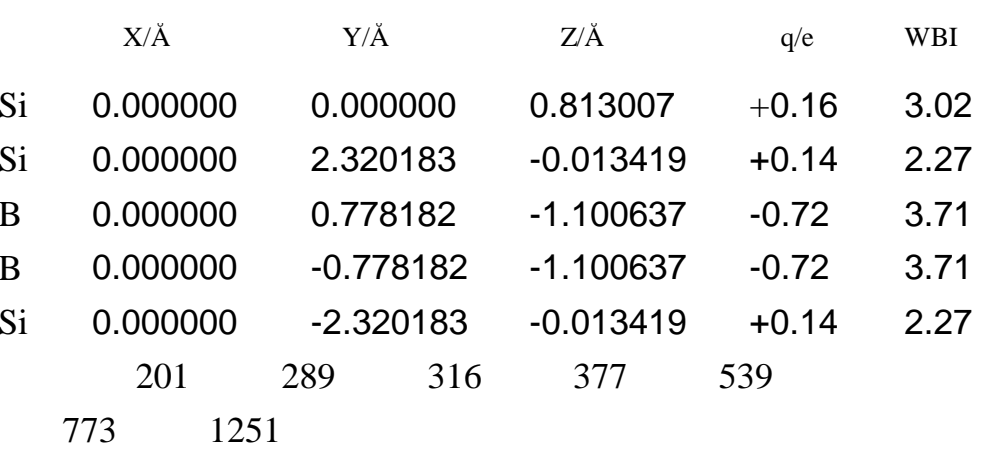

$\begin{array}{lrrrrc}\text { Atom } & 1 & 2 & 3 & 4 & 5 \\ -\cdots & -1 & - & ---- & --- & \\ \text { 1. Si } & 0.0000 & 0.6699 & 0.8379 & 0.8379 & 0.6699 \\ \text { 2. Si } & 0.6699 & 0.0000 & 1.3770 & 0.0676 & 0.1588 \\ \text { 3. B } & 0.8379 & 1.3770 & 0.0000 & 1.4255 & 0.0676 \\ \text { 4. B } & 0.8379 & 0.0676 & 1.4255 & 0.0000 & 1.3770 \\ \text { 5. Si } & 0.6699 & 0.1588 & 0.0676 & 1.3770 & 0.0000\end{array}$

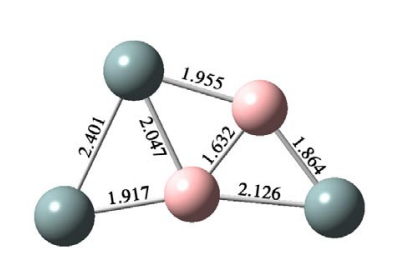

\begin{tabular}{|c|c|c|c|c|c|}
\hline & $\mathrm{X} / \mathrm{A}$ & Y/Ă & Z/Ă & q/e & WBI \\
\hline B & 1.193054 & -0.626877 & 0.000000 & -0.79 & 3.69 \\
\hline B & 0.000000 & 0.487215 & 0.000000 & -0.97 & 3.90 \\
\hline $\mathrm{Si}$ & -0.565703 & -1.479990 & 0.000000 & +0.29 & 2.79 \\
\hline $\mathrm{Si}$ & -1.917327 & 0.504856 & 0.000000 & +0.16 & 2.30 \\
\hline $\mathrm{Si}$ & 2.056939 & 1.025014 & 0.000000 & +0.32 & 2.21 \\
\hline
\end{tabular}

Vibrational frequencies $/ \mathrm{cm}^{-1} 169$

685

$214 \quad 236 \quad 339$

393 509

$\mathrm{HF}=-918.21387$

Wiberg bond Index matrix

$\begin{array}{lllllll}\text { Atom } & 1 & 2 & 3 & 4 & 5 \\ & & & & & 4 & \\ \text { 1. } & \text { B } & 0.0000 & 1.1969 & 0.9859 & 0.1679 & 1.3389 \\ \text { 2. } & \text { B } & 1.1969 & 0.0000 & 0.8521 & 1.2408 & 0.6121 \\ \text { 3. Si } & 0.9859 & 0.8521 & 0.0000 & 0.7921 & 0.1558 \\ \text { 4. Si } & 0.1679 & 1.2408 & 0.7921 & 0.0000 & 0.0997 \\ \text { 5. Si } & 1.3389 & 0.6121 & 0.1558 & 0.0997 & 0.0000\end{array}$


(3). $\mathrm{C}_{\mathrm{s}} \mathrm{B}_{2} \mathrm{Si}_{2} \mathrm{Si}^{-}$

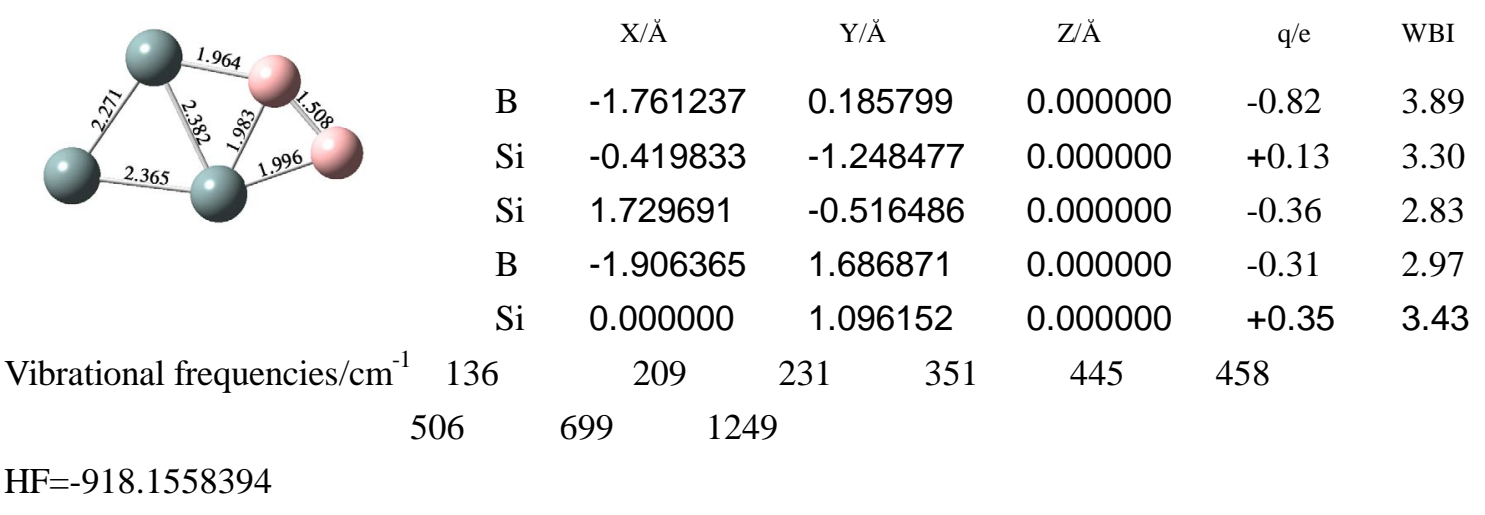

Wiberg bond Index matrix

$\begin{array}{lllllll}\text { Atom } & 1 & 2 & 3 & 4 & 5 \\ & & & & & \\ \text { 1. B } & 0.0000 & 1.0280 & 0.1772 & 1.7965 & 0.8848 \\ \text { 2. Si } & 1.0280 & 0.0000 & 1.4557 & 0.0605 & 0.7554 \\ \text { 3. Si } & 0.1772 & 1.4557 & 0.0000 & 0.2597 & 0.9374 \\ \text { 4. B } & 1.7965 & 0.0605 & 0.2597 & 0.0000 & 0.8512 \\ \text { 5. Si } & 0.8848 & 0.7554 & 0.9374 & 0.8512 & 0.0000\end{array}$

(4). $\mathrm{C}_{\mathrm{s}} \mathrm{B}_{2} \mathrm{Si}_{2} \mathrm{Si}^{-}$

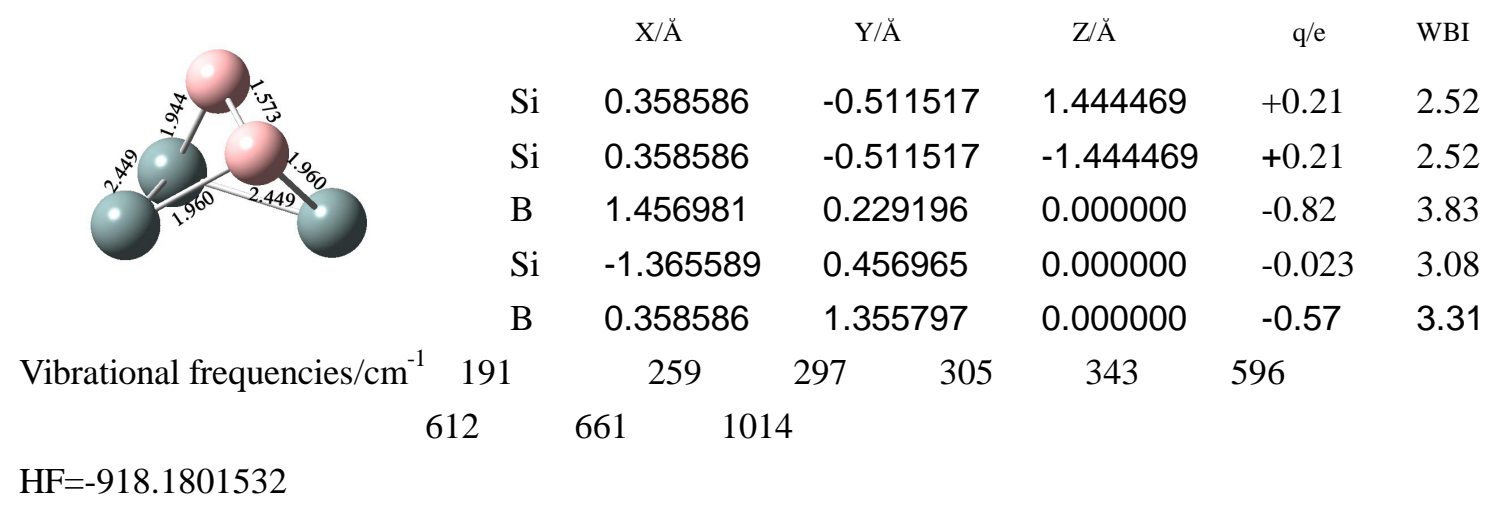

Wiberg bond Index matrix

$\begin{array}{lllllll}\text { Atom } & 1 & 2 & 3 & 4 & 5 \\ & & & & & \\ \text { 1. Si } & 0.0000 & 0.4146 & 0.9614 & 0.7346 & 0.4123 \\ \text { 2. Si } & 0.4146 & 0.0000 & 0.9614 & 0.7346 & 0.4123 \\ \text { 3. B } & 0.9614 & 0.9614 & 0.0000 & 0.5163 & 1.3917 \\ \text { 4. Si } & 0.7346 & 0.7346 & 0.5163 & 0.0000 & 1.0924 \\ \text { 5. B } & 0.4123 & 0.4123 & 1.3917 & 1.0924 & 0.0000\end{array}$


(5). $\mathrm{D}_{3 \mathrm{~h}} \mathrm{~B}_{2} \mathrm{Si}_{2} \mathrm{Si}^{-}$

\begin{tabular}{|c|c|c|c|c|c|c|}
\hline & & $\mathrm{X} / \mathrm{A}$ & $\mathrm{Y} / \mathrm{A}$ & Z/Ă & $q / e$ & WBI \\
\hline & $\mathrm{Si}$ & 0.000000 & 1.628679 & 0.000000 & +0.33 & 2.73 \\
\hline & $\mathrm{Si}$ & 1.410477 & -0.814339 & 0.000000 & +0.33 & 2.73 \\
\hline & $\mathrm{Si}$ & -1.410477 & -0.814339 & 0.000000 & +0.33 & 2.73 \\
\hline & B & 0.000000 & 0.000000 & 1.129833 & -0.99 & 3.34 \\
\hline & B & 0.000000 & 0.000000 & -1.129833 & -0.99 & 3.34 \\
\hline Vibrational frequencies $/ \mathrm{cm}^{-1}$ & 240 & 240 & 366 & 366 & & \\
\hline 67 & & 676 & & & & \\
\hline
\end{tabular}

Wiberg bond Index matrix

$\begin{array}{ccccccc}\text { Atom } & 1 & 2 & 3 & 4 & 5 \\ \text { - } & - & & & 4 & & \\ \text { 1. Si } & 0.0000 & 0.3927 & 0.3927 & 0.9702 & 0.9702 \\ \text { 2. Si } & 0.3927 & 0.0000 & 0.3927 & 0.9702 & 0.9702 \\ \text { 3. Si } & 0.3927 & 0.3927 & 0.0000 & 0.9702 & 0.9702 \\ \text { 4. } & \text { B } & 0.9702 & 0.9702 & 0.9702 & 0.0000 & 0.4301 \\ \text { 5. } & \text { B } & 0.9702 & 0.9702 & 0.9702 & 0.4301 & 0.0000\end{array}$

(6). $\mathrm{D}^{*}{ }_{\mathrm{h}} \mathrm{B}_{2} \mathrm{Si}_{2} \mathrm{Si}^{-}$

\begin{tabular}{|c|c|c|c|c|c|c|}
\hline & & $\mathrm{X} / \mathrm{A}$ & Y/Ă & Z/Ă & $\mathrm{q} / \mathrm{e}$ & WBI \\
\hline & $\mathrm{Si}$ & 0.000000 & 0.000000 & 3.665516 & +0.31 & 2.24 \\
\hline 1.8331 .833 & $\mathrm{Si}$ & 0.000000 & 0.000000 & 0.000000 & +0.72 & 3.74 \\
\hline & $\mathrm{Si}$ & 0.000000 & 0.000000 & -3.665516 & +0.31 & 2.24 \\
\hline & B & 0.000000 & 0.000000 & 1.832961 & -1.17 & 3.62 \\
\hline & B & 0.000000 & 0.000000 & -1.832961 & -1.17 & 3.62 \\
\hline ibrational frequencies $/ \mathrm{cm}^{-1}$ & 42 & 44 & 110 & 188 & 226 & \\
\hline 313 & 523 & 1114 & & & & \\
\hline
\end{tabular}

Wiberg bond Index matrix

$\begin{array}{ccccccc}\text { Atom } & 1 & 2 & 3 & 4 & 5 \\ \text {--- } & -1 & --- & ---- & ---- & --- & \\ \text { 1. Si } & 0.0000 & 0.2242 & 0.1049 & 1.8494 & 0.0574 \\ \text { 2. Si } & 0.2242 & 0.0000 & 0.2242 & 1.6477 & 1.6477 \\ \text { 3. Si } & 0.1049 & 0.2242 & 0.0000 & 0.0574 & 1.8494 \\ \text { 4. } & \text { B } & 1.8494 & 1.6477 & 0.0574 & 0.0000 & 0.0687 \\ \text { 5. B } & 0.0574 & 1.6477 & 1.8494 & 0.0687 & 0.0000\end{array}$




\section{2. $\mathrm{C}_{2 \mathrm{v}} \mathrm{B}_{3} \mathrm{Si}_{2} \mathrm{Si}^{-}$}

\section{(1). $\mathrm{C}_{2 \mathrm{v}} \mathrm{B}_{3} \mathrm{Si}_{2} \mathrm{Si}^{-}$}

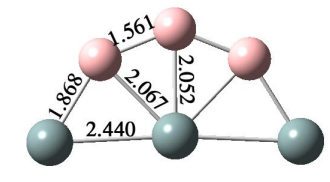

$\begin{array}{cccccc} & X / \AA ̆ & Y / A ̆ & Z / A ̆ & q / e & \text { WBI } \\ \text { Si } & 0.000000 & 0.000000 & 0.382866 & +0.45 & 3.43 \\ \mathrm{~B} & 0.000000 & 1.450315 & -1.090422 & -0.76 & 3.88 \\ \mathrm{~B} & 0.000000 & 0.000000 & -1.668657 & -0.20 & 3.59 \\ \mathrm{~B} & 0.000000 & -1.450315 & -1.090422 & -0.76 & 3.88 \\ \mathrm{Si} & 0.000000 & -2.437030 & 0.495978 & +0.13 & 2.71 \\ \mathrm{Si} & 0.000000 & 2.437030 & 0.495978 & +0.13 & 2.71\end{array}$

Vibrational frequencies $/ \mathrm{cm}^{-1} 126$ $\begin{array}{llllll}464 & 580 & 655 & 734 & 1092 & 1296\end{array}$

$\mathrm{HF}=-943.0628808$

Wiberg bond Index matrix

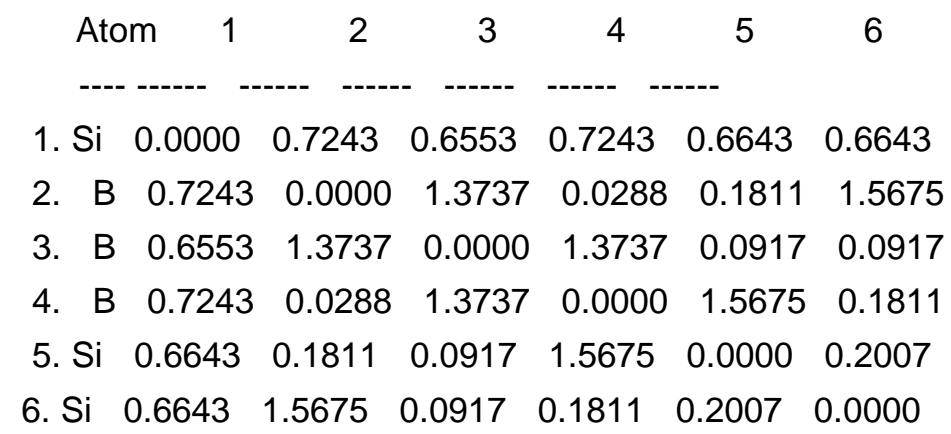

\section{(2). $\mathrm{C}_{2 \mathrm{v}} \mathrm{B}_{3} \mathrm{Si}_{2} \mathrm{Si}^{-}$}

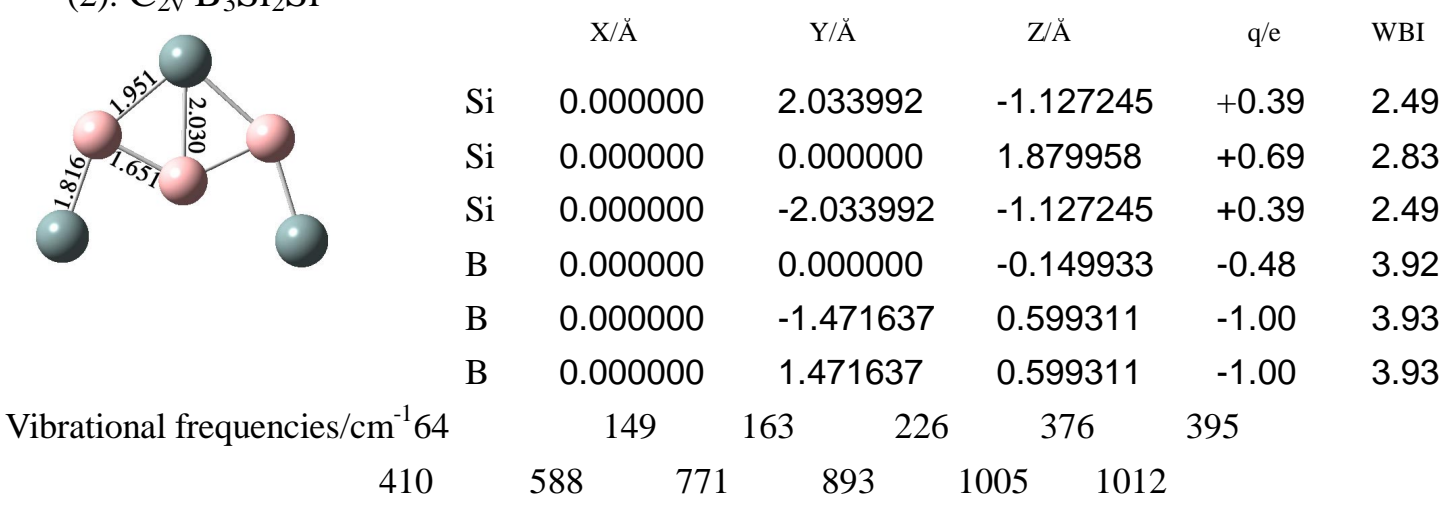

$\mathrm{HF}=-943.0366644$

Wiberg bond Index matrix

\begin{tabular}{|c|c|c|c|c|c|c|}
\hline \multicolumn{2}{|c|}{ Atom } & 2 & 3 & & \multirow[t]{2}{*}{6} \\
\hline & & 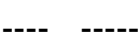 & 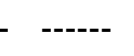 & 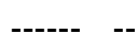 & & \\
\hline Si & 0000 & 0. & 9 & 0 & & 90 \\
\hline Si & 0.1485 & 0.0000 & 485 & 0.7 & 79 & 79 \\
\hline $\mathrm{Si}$ & 0.1699 & 0.1 & 0.0000 & 0.4396 & 1.5996 & 0.1302 \\
\hline B & 0.439 & 0.7179 & 0.4396 & 0.0000 & 1.1635 & 1.1635 \\
\hline$\checkmark$ & 0.13 & $0 . \subseteq$ & 1.5996 & 5 & 0 & 0 \\
\hline B & 1.5996 & 0.9079 & 0.1302 & 1.1635 & 0.1284 & 0.000 \\
\hline
\end{tabular}


(3). $\mathrm{C}_{3 \mathrm{v}} \mathrm{B}_{3} \mathrm{Si}_{2} \mathrm{Si}^{-}$

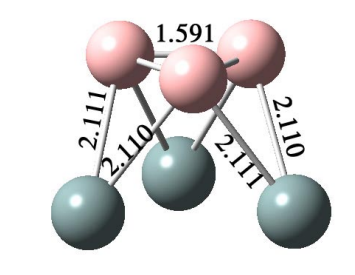

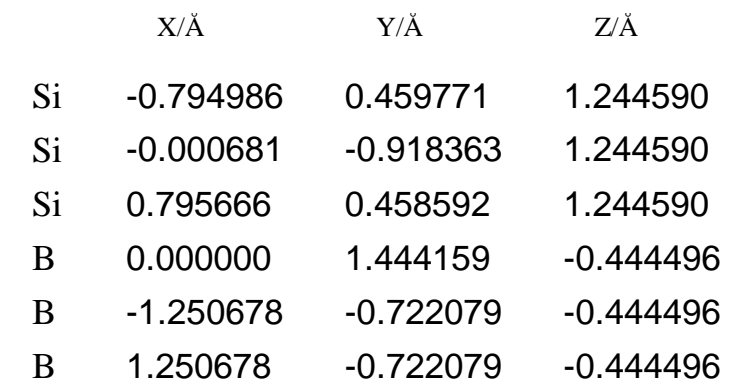

Vibrational frequencies $/ \mathrm{cm}^{-1} 77$ 463

\begin{tabular}{lllllll} 
& 85 & \multicolumn{2}{c}{238} & 239 & 244 & 322 \\
464 & 577 & 912 & 912 & 1136 &
\end{tabular}

$\mathrm{HF}=-943.02173742$

(4). $\mathrm{C}_{1} \mathrm{~B}_{3} \mathrm{Si}_{2} \mathrm{Si}^{-}$

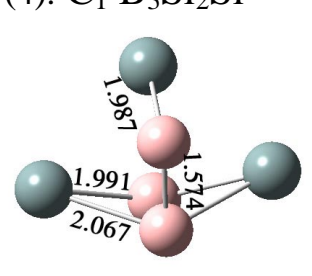

\begin{tabular}{llllll} 
& \multicolumn{1}{c}{ X/Ă } & Y/Ă & Z/Ă & q/e & WBI \\
$\mathrm{Si}$ & 1.769731 & -0.384787 & -0.125054 & +0.31 & 2.58 \\
$\mathrm{~B}$ & -0.001659 & 0.109879 & 1.424237 & -0.46 & 3.63 \\
$\mathrm{~B}$ & 0.000427 & -1.205144 & 0.559498 & -0.62 & 3.91 \\
$\mathrm{~B}$ & 0.000191 & -0.629673 & -1.004423 & -0.76 & 3.77 \\
$\mathrm{Si}$ & -1.769399 & -0.384993 & -0.125547 & +0.31 & 2.58 \\
$\mathrm{Si}$ & 0.000040 & 1.385829 & -0.099153 & +0.22 & 3.00
\end{tabular}

Vibrational frequencies $/ \mathrm{cm}^{-1} 154$ 490

$\mathrm{HF}=-943.0389037$

Wiberg bond Index matrix

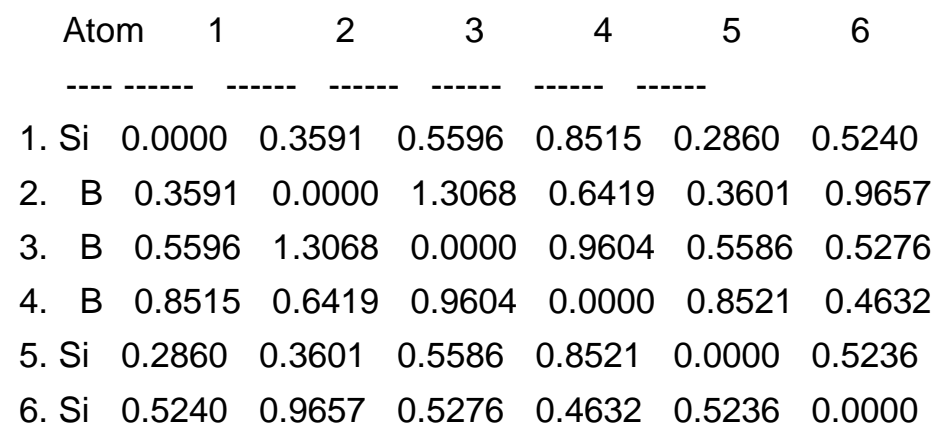

(5). $\mathrm{C}_{\mathrm{s}} \mathrm{B}_{3} \mathrm{Si}_{2} \mathrm{Si}^{-}$

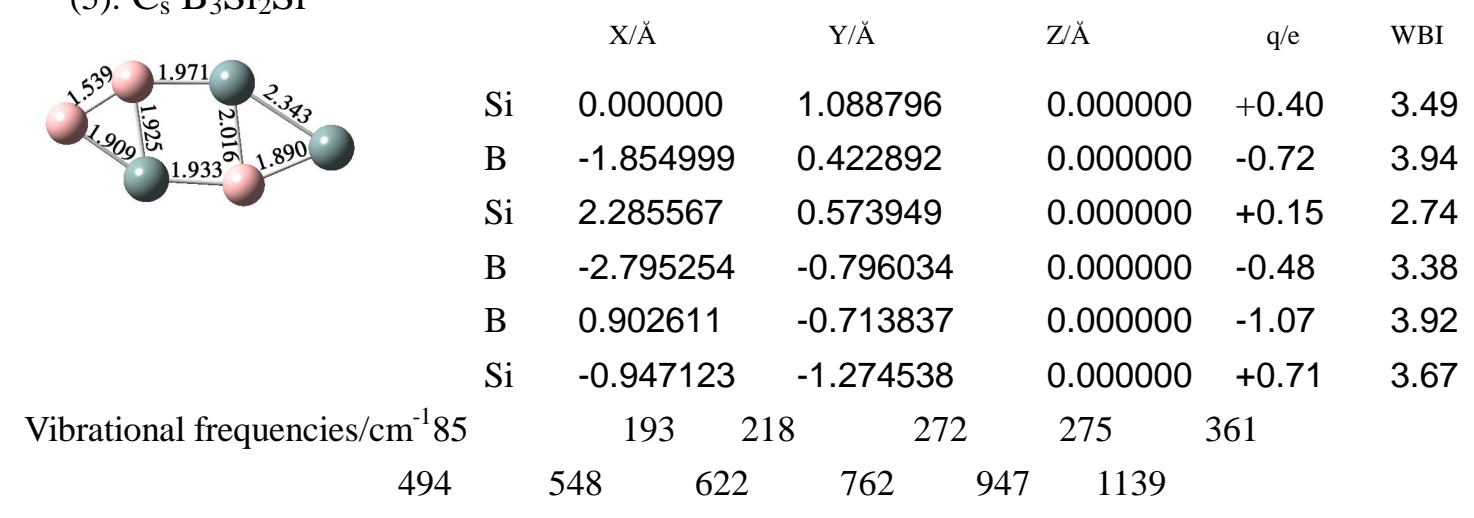


$\mathrm{HF}=-942.9814675$

Wiberg bond Index matrix

\begin{tabular}{lrrrrrc}
\multicolumn{1}{l}{ Atom } & 1 & 2 & 3 & 4 & 5 & 6 \\
1. Si & 0.0000 & 1.0075 & 0.9356 & 0.1350 & 1.0741 & 0.3335 \\
2. B & 1.0075 & 0.0000 & 0.1205 & 1.7256 & 0.0898 & 0.9935 \\
3. Si & 0.9356 & 0.1205 & 0.0000 & 0.1462 & 1.4845 & 0.0567 \\
4. B & 0.1350 & 1.7256 & 0.1462 & 0.0000 & 0.1752 & 1.1947 \\
5. B & 1.0741 & 0.0898 & 1.4845 & 0.1752 & 0.0000 & 1.0963 \\
6. Si & 0.3335 & 0.9935 & 0.0567 & 1.1947 & 1.0963 & 0.0000
\end{tabular}

(6). $\mathrm{C}_{\mathrm{s}} \mathrm{B}_{3} \mathrm{Si}_{2} \mathrm{Si}^{-}$

$\begin{array}{lllllll}\mathrm{X} / \overline{\mathrm{A}} & \mathrm{Y} / \mathrm{A} & \mathrm{Z} / \mathrm{A} & \mathrm{q} / \mathrm{e} & \mathrm{WBI} \\ \mathrm{Si} & 0.003339 & 1.297749 & 0.308467 & +0.37 & 2.51 \\ \mathrm{Si} & -2.034146 & -0.095507 & -0.253159 & +0.16 & 2.71 \\ \mathrm{~B} & -0.001287 & -0.442760 & -0.592951 & -0.90 & 3.96 \\ \mathrm{~B} & 0.788945 & -1.325086 & 0.574621 & -0.39 & 3.59 \\ & \mathrm{~B} & -0.792167 & -1.323180 & 0.574027 & -0.39 & 3.60 \\ & \mathrm{Si} & 2.032418 & -0.098305 & -0.253772 & +0.16 & 2.71\end{array}$

\begin{tabular}{cccccccc} 
Vibrational frequencies $/ \mathrm{cm}^{-1} 150$ & \multicolumn{1}{c}{$\begin{array}{l}187 \\
494\end{array}$} & 667 & 680 & 835 & 915 & 1134 &
\end{tabular}

$\mathrm{HF}=-943.0556757$

Wiberg bond Index matrix

\begin{tabular}{|c|c|c|c|c|c|c|}
\hline \multicolumn{2}{|c|}{ Atom } & 2 & 3 & 4 & & \\
\hline & ---- & - & ------ & -- & --- & \\
\hline $\mathrm{Si}$ & 0.0000 & 0.5883 & 0.8627 & 0.2336 & 0.2330 & 0.5931 \\
\hline 2. $\mathrm{Si}$ & 0.5883 & 0.0000 & 0.6353 & 0.0858 & 1.1187 & 0.2830 \\
\hline 3. $\mathrm{B}$ & 0.8627 & 0.6353 & 0.0000 & 0.9118 & 0.9132 & 0.6345 \\
\hline 4. $B$ & 0.2336 & 0.0858 & 0.9118 & 0.0000 & 1.2448 & 1.1180 \\
\hline 5. B & 0.2330 & 1.1187 & 0.9132 & 1.2448 & 0.0000 & 0.0858 \\
\hline $6 . \varsigma$ & .5931 & 0.2830 & 0.6345 & 1.1180 & 0.0858 & 0.0000 \\
\hline
\end{tabular}

(7). $\mathrm{C}_{\mathrm{s}} \mathrm{B}_{3} \mathrm{Si}_{2} \mathrm{Si}^{-}$

$\begin{array}{lllllll}\mathrm{X} / \overline{\mathrm{A}} & \mathrm{Y} / \mathrm{A} & \mathrm{Z} / \overline{\mathrm{A}} & \mathrm{q} / \mathrm{e} & \text { WBI } \\ & \mathrm{Si} & 1.167978 & -1.772516 & -0.011531 & +0.45 & 2.44 \\ \mathrm{Si} & 1.167964 & 1.772524 & -0.011530 & +0.45 & 2.44 \\ \mathrm{Si} & -2.145165 & -0.000008 & 0.106355 & +0.50 & 2.53 \\ \mathrm{~B} & -0.519432 & -0.920719 & -0.406165 & -0.82 & 3.81 \\ \mathrm{~B} & 0.504699 & 0.000003 & 0.579109 & -0.76 & 3.84 \\ \mathrm{~B} & -0.519443 & 0.920717 & -0.406169 & -0.82 & 3.81\end{array}$

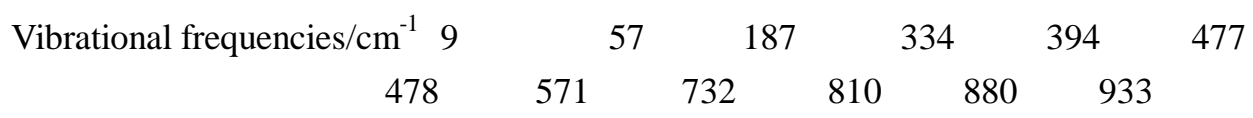

$H F=-943.0415323$ 
Wiberg bond Index matrix

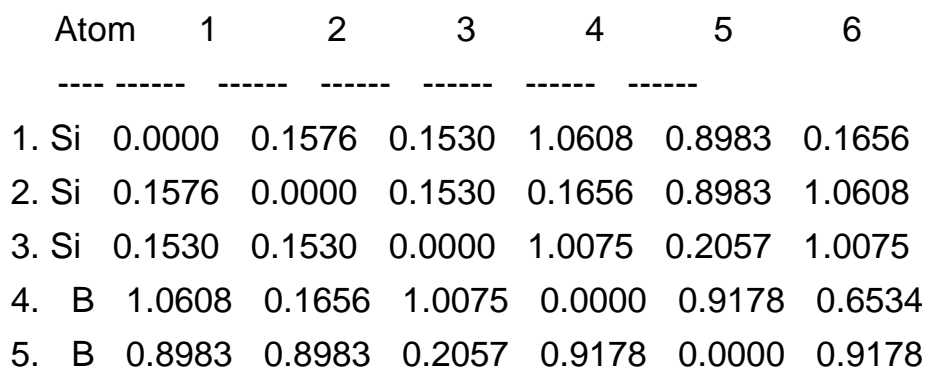

6. $\quad$ B $\quad 0.1656 \quad 1.0608 \quad 1.0075 \quad 0.6534 \quad 0.9178 \quad 0.0000$

(8). $\mathrm{C}_{\mathrm{s}} \mathrm{B}_{3} \mathrm{Si}_{2} \mathrm{Si}^{-}$

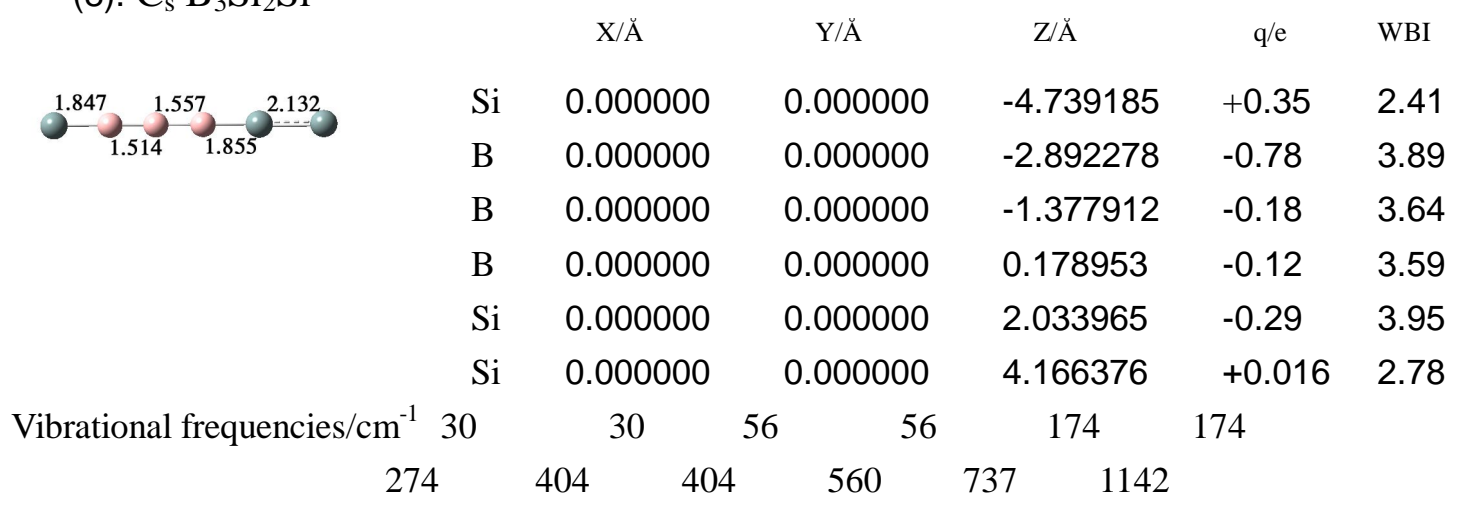

$\mathrm{HF}=-942.9617071$

Wiberg bond Index matrix

\begin{tabular}{|c|c|c|c|c|c|c|}
\hline \multirow[t]{2}{*}{ Atom } & $n$ & 2 & 3 & 4 & 5 & 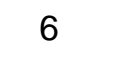 \\
\hline & ----- & $\ldots$ & -- & ------ $\quad----$ & & \\
\hline & 0.0000 & 1.67 & & 0. & & \\
\hline B & 1.6710 & 0.0 & 1 & & & \\
\hline B & 0.4171 & 1.7624 & .0000 & & & 0.0123 \\
\hline$B$ & 0.0877 & 0.2637 & 1.3795 & 0.0 & 1.5968 & 0.2599 \\
\hline & .0721 & .0348 & 3 & & 00 & 2.1 \\
\hline & 09 & .1606 & .0123 & 599 & 2.1815 & 0.0000 \\
\hline
\end{tabular}

